\title{
Review Article \\ Quantum Entanglement: Separability, Measure, Fidelity of Teleportation, and Distillation
}

\author{
Ming Li, ${ }^{1}$ Shao-Ming Fei, ${ }^{2,3}$ and Xianqing Li-Jost ${ }^{3}$ \\ ${ }^{1}$ College of Mathematics and Computational Science, China University of Petroleum, \\ 257061 Dongying, China \\ ${ }^{2}$ Department of Mathematics, Capital Normal University, 100037 Beijing, China \\ ${ }^{3}$ Max-Planck-Institute for Mathematics in the Sciences, 04103 Leipzig, Germany \\ Correspondence should be addressed to Ming Li, liming3737@163.com
}

Received 29 August 2009; Accepted 2 December 2009

Academic Editor: NaiHuan Jing

Copyright (C) 2010 Ming Li et al. This is an open access article distributed under the Creative Commons Attribution License, which permits unrestricted use, distribution, and reproduction in any medium, provided the original work is properly cited.

\begin{abstract}
Quantum entanglement plays crucial roles in quantum information processing. Quantum entangled states have become the key ingredient in the rapidly expanding field of quantum information science. Although the nonclassical nature of entanglement has been recognized for many years, considerable efforts have been taken to understand and characterize its properties recently. In this review, we introduce some recent results in the theory of quantum entanglement. In particular separability criteria based on the Bloch representation, covariance matrix, normal form and entanglement witness, lower bounds, subadditivity property of concurrence and tangle, fully entangled fraction related to the optimal fidelity of quantum teleportation, and entanglement distillation will be discussed in detail.
\end{abstract}

\section{Introduction}

Entanglement is the characteristic trait of quantum mechanics, and it reflects the property that a quantum system can simultaneously appear in two or more different states [1]. This feature implies the existence of global states of composite system which cannot be written as a product of the states of individual subsystems. This phenomenon [2], now known as "quantum entanglement," plays crucial roles in quantum information processing [3]. Quantum entangled states have become the key ingredient in the rapidly expanding field of quantum information science, with remarkable prospective applications such as quantum computation [3, 4], quantum teleportation [5-9], dense coding [10], quantum cryptographic schemes [11-13], entanglement swapping [14-18], and remote states preparation (RSP) [1924]. All such effects are based on entanglement and have been demonstrated in pioneering experiments. 
It has become clear that entanglement is not only the subject of philosophical debates, but also a new quantum resource for tasks which cannot be performed by means of classical resources. Although considerable efforts have been taken to understand and characterize the properties of quantum entanglement recently, the physical character and mathematical structure of entangled states have not been satisfactorily understood yet $[25,26]$. In this review we mainly introduce some recent results related to our researches on several basic questions in this subject.

\section{(1) Separability of Quantum States}

We first discuss the separability of a quantum states; namely, for a given quantum state, how we can know whether or not it is entangled.

For pure quantum states, there are many ways to verify the separability. For instance, for a bipartite pure quantum state the separability is easily determined in terms of its Schmidt numbers. For multipartite pure states, the generalized concurrence given in [27] can be used to judge if the state is separable or not. In addition separable states must satisfy all possible Bell inequalities [28].

For mixed states we still have no general criterion. The well-known PPT (partial positive transposition) criterion was proposed by Peres in 1996 [29]. It says that for any bipartite separable quantum state the density matrix must be positive under partial transposition. By using the method of positive maps Horodecki et al. [30] showed that the Peres' criterion is also sufficient for $2 \times 2$ and $2 \times 3$ bipartite systems. And for higher dimensional states, the PPT criterion is only necessary. Horodecki [31] has constructed some classes entangled states with positive partial transposes for $3 \times 3$ and $2 \times 4$ systems. States of this kind are said to be bound entangled (BE). Another powerful operational criterion is the realignment criterion $[32,33]$. It demonstrates a remarkable ability to detect many bound entangled states and even genuinely tripartite entanglement [34]. Considerable efforts have been made in finding stronger variants and multipartite generalizations for this criterion [35-39]. It was shown that PPT criterion and realignment criterion are equivalent to the permutations of the density matrix's indices [34]. Another important criterion for separability is the reduction criterion [40,41]. This criterion is equivalent to the PPT criterion for $2 \times N$ composite systems. Although it is generally weaker than the PPT, the reduction criteria have tight relation to the distillation of quantum states.

There are also some other necessary criteria for separability. Nielsen and Kempe [42] presented a necessary criterion called majorization: the decreasing ordered vector of the eigenvalues for $\rho$ is majorized by that of $\rho^{A_{1}}$ or $\rho^{A_{2}}$ alone for a separable state. That is, if a state $\rho$ is separable, then $\lambda_{\rho}^{\downarrow}<\lambda_{\rho^{A_{1}}}^{\downarrow}, \lambda_{\rho}^{\downarrow}<\lambda_{\rho^{A_{2}}}^{\downarrow}$. Here $\lambda_{\rho}^{\downarrow}$ denotes the decreasing ordered vector of the eigenvalues of $\rho$. A $d$-dimensional vector $x^{\downarrow}$ is majorized by $y^{\downarrow}, x^{\downarrow} \prec y^{\downarrow}$, if $\sum_{j=1}^{k} x_{j}^{\downarrow} \leq \sum_{j=1}^{k} y_{j}^{\downarrow}$ for $k=1, \ldots, d-1$ and the equality holds for $k=d$. Zeros are appended to the vectors $\lambda_{\rho^{A_{1}, A_{2}}}^{\downarrow}$ such that their dimensions are equal to the one of $\lambda_{\rho}^{\downarrow}$.

In [31], another necessary criterion called range criterion was given. If a bipartite state $\rho$ acting on the space $\mathscr{\ell}_{A} \otimes \mathscr{\ell}_{B}$ is separable, then there exists a family of product vectors $\psi_{i} \otimes \phi_{i}$ such that (i) they span the range of $\rho$; (ii) the vector $\left\{\psi_{i} \otimes \phi_{i}^{*}\right\}_{i=1}^{k}$ spans the range of $\rho^{T_{B}}$, where $*$ denotes complex conjugation in the basis in which partial transposition was performed and $\rho^{T_{B}}$ is the partially transposed matrix of $\rho$ with respect to the subspace $B$. In particular, any of the vectors $\psi_{i} \otimes \phi_{i}^{*}$ belongs to the range of $\rho$. 
Recently, some elegant results for the separability problem have been derived. In [4345], a separability criteria based on the local uncertainty relations (LURs) was obtained. The authors show that, for any separable state $\rho \in \mathscr{l}_{A} \otimes \mathscr{l}_{B}$,

$$
1-\sum_{k}\left\langle G_{k}^{A} \otimes G_{k}^{B}\right\rangle-\frac{1}{2}\left\langle G_{k}^{A} \otimes I-I \otimes G_{k}^{B}\right\rangle^{2} \geq 0
$$

where $G_{k}^{A}$ or $G_{k}^{B}$ are arbitrary local orthogonal and normalized operators (LOOs) in $\mathscr{d}_{A} \otimes \mathfrak{d}_{B}$. This criterion is strictly stronger than the realignment criterion. Thus more bound entangled quantum states can be recognized by the LUR criterion. The criterion is optimized in [46] by choosing the optimal LOOs. In [47] a criterion based on the correlation matrix of a state has been presented. The correlation matrix criterion is shown to be independent of PPT and realignment criterion [48], that is, there exist quantum states that can be recognized by correlation criterion while the PPT and realignment criterion fail. The covariance matrix of a quantum state is also used to study separability in [49]. It has been shown that the LUR criterion, including the optimized one, can be derived from the covariance matrix criterion [50].

\section{(2) Measure of Quantum Entanglement}

One of the most difficult and fundamental problems in entanglement theory is to quantify entanglement. The initial idea to quantify entanglement was connected with its usefulness in terms of communication [51]. A good entanglement measure has to fulfill some conditions [52]. For bipartite quantum systems, we have several good entanglement measures such as Entanglement of Formation (EOF), Concurrence, and Tangle ctc. For two-qubit systems it has been proved that EOF is a monotonically increasing function of the concurrence and an elegant formula for the concurrence was derived analytically by Wootters [53]. However with the increasing dimensions of the subsystems the computation of EOF and concurrence become formidably difficult. A few explicit analytic formulae for EOF and concurrence have been found only for some special symmetric states [54-58].

The first analytic lower bound of concurrence for arbitrary dimensional bipartite quantum states was derived by Mintert et al. in [59]. By using the positive partial transposition (PPT) and realignment separability criterion, analytic lower bounds on EOF and concurrence for any dimensional mixed bipartite quantum states have been derived in $[60,61]$. These bounds are exact for some special classes of states and can be used to detect many bound entangled states. In [62] another lower bound on EOF for bipartite states has been presented from a new separability criterion [63]. A lower bound of concurrence based on local uncertainty relations (LURs) criterion is derived in [64]. This bound is further optimized in [46]. The lower bound of concurrence for tripartite systems has been studied in [65]. In $[66,67]$ the authors presented lower bounds of concurrence for bipartite systems by considering the "two-qubit" entanglement of bipartite quantum states with arbitrary dimensions. It has been shown that this lower bound has a tight relationship with the distillability of bipartite quantum states. Tangle is also a good entanglement measure that has a close relation with concurrence, as it is defined by the square of the concurrence for a pure state. It is also meaningful to derive tight lower and upper bounds for tangle [68].

In [69] Mintert et al. proposed an experimental method to measure the concurrence directly by using joint measurements on two copies of a pure state. Then Walborn et al. 
presented an experimental determination of concurrence for two-qubit states [70,71], where only one-setting measurement is needed, but two copies of the state have to be prepared in every measurement. In [72] another way of experimental determination of concurrence for two-qubit and multiqubit states has been presented, in which only one copy of the state is needed in every measurement. To determine the concurrence of the two-qubit state used in [70, 71], also one-setting measurement is needed, which avoids the preparation of the twin states or the imperfect copy of the unknown state, and the experimental difficulty is dramatically reduced.

\section{(3) Fidelity of Quantum Teleportation and Distillation}

Quantum teleportation, or entanglement-assisted teleportation, is a technique used to transfer information on a quantum level, usually from one particle (or series of particles) to another particle (or series of particles) in another location via quantum entanglement. It does not transport energy or matter, nor does it allow communication of information at super luminal (faster than light) speed.

In [5-7], Bennett et al. first presented a protocol to teleport an unknown qubit state by using a pair of maximally entangled pure qubit state. The protocol is generalized to transmit high-dimensional quantum states $[8,9]$. The optimal fidelity of teleportation is shown to be determined by the fully entangled fraction of the entangled resource which is generally a mixed state. Nevertheless similar to the estimation of concurrence, the computation of the fully entangled fraction for a given mixed state is also very difficult.

The distillation protocol has been presented to get maximally entangled pure states from many entangled mixed states by means of local quantum operations and classical communication (LQCC) between the parties sharing the pairs of particles in this mixed state [73-76]. Bennett et al. first derived a protocol to distill one maximally entangled pure Bell state from many copies of not maximally entangled quantum mixed states in [73] in 1996. The protocol is then generalized to distill any bipartite quantum state with higher dimension by M. Horodecki and P. Horodecki in 1999 [77]. It is proven that a quantum state can be always distilled if it violates the reduced matrix separability criterion [77].

This review mainly contains three parts. In Section 2 we investigate the separability of quantum states. We first introduce several important separability criteria. Then we discuss the criteria by using the Bloch representation of the density matrix of a quantum state. We also study the covariance matrix of a quantum density matrix and derive separability criterion for multipartite systems. We investigate the normal forms for multipartite quantum states at the end of this section and show that the normal form can be used to improve the power of these criteria. In Section 3 we mainly consider the entanglement measure concurrence. We investigate the lower and upper bounds of concurrence for both bipartite and multipartite systems. We also show that the concurrence and tangle of two entangled quantum states will be always larger than that of one, even if both of the two states are bound entangled (not distillable). In Section 4 we study the fully entangled fraction of an arbitrary bipartite quantum state. We derive precise formula of fully entangled fraction for two-qubit system. For bipartite system with higher dimension we obtain tight upper bounds which can not only be used to estimate the optimal teleportation fidelity but also help to improve the distillation protocol. We further investigate the evolution of the fully entangled fraction when one of the bipartite system undergoes a noisy channel. We give a summary and conclusion in the last section. 


\section{Separability Criteria and Normal Form}

A multipartite pure quantum state $\rho_{12 \cdots N} \in \mathscr{\ell}_{1} \otimes \mathfrak{h}_{2} \otimes \cdots \otimes \mathscr{\ell}_{N}$ is said to be fully separable if it can be written as

$$
\rho_{12 \cdots N}=\rho_{1} \otimes \rho_{2} \otimes \cdots \otimes \rho_{N},
$$

where $\rho_{1}$ and $\rho_{2}, \ldots, \rho_{N}$ are reduced density matrices defined as $\rho_{1}=\operatorname{Tr}_{23 \cdots N}\left[\rho_{12 \cdots N}\right], \rho_{2}=$ $\operatorname{Tr}_{13 \cdots N}\left[\rho_{12 \cdots N}\right], \ldots, \rho_{N}=\operatorname{Tr}_{12 \cdots N-1}\left[\rho_{12 \cdots N}\right]$. This is equivalent to the condition

$$
\rho_{12 \cdots N}=\left|\psi_{1}\right\rangle\left\langle\psi_{1}|\otimes| \phi_{2}\right\rangle\left\langle\phi_{2}|\otimes \cdots \otimes| \mu_{N}\right\rangle\left\langle\mu_{N}\right|,
$$

where $\left|\psi_{1}\right\rangle \in \mathscr{H}_{1},\left|\phi_{2}\right\rangle \in \mathscr{H}_{2}, \ldots,\left|\mu_{N}\right\rangle \in \mathscr{H}_{N}$.

A multipartite quantum mixed state $\rho_{12 \cdots N} \in \mathscr{H}_{1} \otimes \mathscr{\ell}_{2} \otimes \cdots \otimes \mathscr{H}_{N}$ is said to be fully separable if it can be written as

$$
\rho_{12 \cdots N}=\sum_{i} q_{i} \rho_{i}{ }^{1} \otimes \rho_{i}{ }^{2} \otimes \cdots \otimes \rho_{i}{ }^{N}
$$

where $\rho_{i}{ }^{1}, \rho_{i}{ }^{2}, \ldots, \rho_{i}{ }^{N}$ are the reduced density matrices with respect to the systems $1,2, \ldots, N$, respectively, $q_{i}>0$, and $\sum_{i} q_{i}=1$. This is equivalent to the condition

$$
\rho_{12 \cdots N}=\sum_{i} p_{i}\left|\psi_{i}^{1}\right\rangle\left\langle\psi_{i}^{1}|\otimes| \phi_{i}^{2}\right\rangle\left\langle\phi_{i}^{2}|\otimes \cdots \otimes| \mu_{i}^{N}\right\rangle\left\langle\mu_{i}^{N}\right|,
$$

where $\left|\psi_{i}^{1}\right\rangle,\left|\phi_{i}^{2}\right\rangle, \ldots,\left|\mu_{i}^{N}\right\rangle$ are normalized pure states of systems $1,2, \ldots, N$, respectively, $\left.p_{i}\right\rangle$ 0 , and $\sum_{i} p_{i}=1$.

For pure states, the definition (2.1) itself is an operational separability criterion. In particular, for bipartite case, there are Schmidt decompositions.

Theorem 2.1 (see Schmidt decomposition in [78]). Suppose that $|\psi\rangle \in \mathscr{l}_{A} \otimes \mathscr{l}_{B}$ is a pure state of a composite system, $A B$, then there exist orthonormal states $\left|i_{A}\right\rangle$ for system $A$ and orthonormal states $\left|i_{B}\right\rangle$ for system $B$ such that

$$
|\psi\rangle=\sum_{i} \lambda_{i}\left|i_{A}\right\rangle\left|i_{B}\right\rangle
$$

where $\lambda_{i}$ are nonnegative real numbers satisfying $\sum_{i} \lambda_{i}{ }^{2}=1$, known as Schmidt coefficients.

$\left|i_{A}\right\rangle$ and $\left|i_{B}\right\rangle$ are called Schmidt bases with respect to $\mathscr{L}_{A}$ and $\mathscr{L}_{B}$. The number of nonzero values $\lambda_{i}$ is called Schmidt number, also known as Schmidt rank, which is invariant under unitary transformations on system $A$ or system $B$. For a bipartite pure state $|\psi\rangle,|\psi\rangle$ is separable if and only if the Schmidt number of $|\psi\rangle$ is one.

For multipartite pure states, one has no such Schmidt decomposition. In [79] it has been verified that any pure three-qubit state $|\Psi\rangle$ can be uniquely written as

$$
|\Psi\rangle=\lambda_{0}|000\rangle+\lambda_{1} e^{i \Psi}|100\rangle+\lambda_{2}|101\rangle+\lambda_{3}|110\rangle+\lambda_{4}|111\rangle
$$


with normalization condition $\lambda_{i} \geq 0,0 \leq \psi \leq \pi$, where $\sum_{i} \mu_{i}=1, \mu_{i} \equiv \lambda_{i}^{2}$. Equation (2.6) is called generalized Schmidt decomposition.

For mixed states it is generally very hard to verify whether a decomposition like (2.3) exists. For a given generic separable density matrix, it is also not easy to find the decomposition (2.3) in detail.

\subsection{Separability Criteria for Mixed States}

In this section we introduce several separability criteria and the relations among themselves. These criteria have also tight relations with lower bounds of entanglement measures and distillation that will be discussed in the next section.

\subsubsection{Partial Positive Transpose Criterion}

The positive partial transpose (PPT) criterion provided by Peres [29] says that if a bipartite state $\rho_{A B} \in \mathscr{L}_{A} \otimes \mathscr{l}_{B}$ is separable, then the new matrix $\rho_{A B}^{T_{B}}$ with matrix elements defined in some fixed product basis as

$$
\left\langle m\left|\left\langle\mu\left|\rho_{A B}^{T_{B}}\right| n\right\rangle\right| v\right\rangle \equiv\left\langle m\left|\left\langle v\left|\rho_{A B}\right| n\right\rangle\right| \mu\right\rangle
$$

is also a density matrix (i.e., it has nonnegative spectrum). The operation $T_{B}$, called partial transpose, just corresponds to the transposition of the indices with respect to the second subsystem $B$. It has an interpretation as a partial time reversal [80].

Afterwards Horodecki et al. showed that Peres' criterion is also sufficient for $2 \times 2$ and $2 \times 3$ bipartite systems [30]. This criterion is now called PPT or Peres-Horodecki (P$\mathrm{H})$ criterion. For high-dimensional states, the P-H criterion is only necessary. Horodecki has constructed some classes of families of entangled states with positive partial transposes for $3 \times 3$ and $2 \times 4$ systems [31]. States of this kind are said to be bound entangled (BE).

\subsubsection{Reduced Density Matrix Criterion}

Cerf et al. [81] and M. Horodecki and P. Horodecki [82], independently, introduced a map $\Gamma: \rho \rightarrow \operatorname{Tr}_{B}\left[\rho_{A B}\right] \otimes I-\rho_{A B}\left(I \otimes \operatorname{Tr}_{A}\left[\rho_{A B}\right]-\rho_{A B}\right)$, which gives rise to a simple necessary condition for separability in arbitrary dimensions, called the reduction criterion. If $\rho_{A B}$ is separable, then

$$
\rho_{A} \otimes I-\rho_{A B} \geq 0, \quad I \otimes \rho_{B}-\rho_{A B} \geq 0,
$$

where $\rho_{A}=\operatorname{Tr}_{B}\left[\rho_{A B}\right], \rho_{B}=\operatorname{Tr}_{A}\left[\rho_{A B}\right]$. This criterion is simply equivalent to the P-H criterion for $2 \times n$ composite systems. It is also sufficient for $2 \times 2$ and $2 \times 3$ systems. In higher dimensions the reduction criterion is weaker than the $\mathrm{P}-\mathrm{H}$ criterion. 


\subsubsection{Realignment Criterion}

There is yet another class of criteria based on linear contractions on product states. They stem from the new criterion discovered in $[33,83]$ called computable cross-norm $(\mathrm{CCN})$ criterion or matrix realignment criterion which is operational and independent on PPT test [29]. If a state $\rho_{A B}$ is separable, then the realigned matrix $\mathcal{R}(\rho)$ with elements $\mathcal{R}(\rho)_{i j, k l}=\rho_{i k, j l}$ has trace norm not greater than one:

$$
\|\mathcal{R}(\rho)\|_{K F} \leq 1
$$

Quite remarkably, the realignment criterion can detect some PPT entangled (bound entangled) states $[33,83]$ and can be used for construction of some nondecomposable maps. It also provides nice lower bound for concurrence [61].

\subsubsection{Criteria Based on Bloch Representations}

Any Hermitian operator on an $N$-dimensional Hilbert space $t h$ can be expressed according to the generators of the special unitary group $S U(N)$ [84]. The generators of $S U(N)$ can be introduced according to the transition-projection operators $P_{j k}=|j\rangle\langle k|$, where $|i\rangle, i=$ $1, \ldots, N$, are the orthonormal eigenstates of a linear Hermitian operator on $\mathscr{t}$. Set

$$
\begin{gathered}
\omega_{l}=-\sqrt{\frac{2}{l(l+1)}}\left(P_{11}+P_{22}+\cdots+P_{l l}-l P_{l+1, l+1}\right), \\
u_{j k}=P_{j k}+P_{k j}, \quad v_{j k}=i\left(P_{j k}-P_{k j}\right),
\end{gathered}
$$

where $1 \leq l \leq N-1$ and $1 \leq j<k \leq N$. We get a set of $N^{2}-1$ operators

$$
\Gamma \equiv\left\{\omega_{l}, \omega_{2}, \ldots, \omega_{N-1}, u_{12}, u_{13}, \ldots, v_{12}, v_{13}, \ldots\right\},
$$

which satisfies the relations

$$
\operatorname{Tr}\left[\lambda_{i}\right]=0, \quad \operatorname{Tr}\left[\lambda_{i} \lambda_{j}\right]=2 \delta_{i j}, \quad \forall \lambda_{i} \in \Gamma,
$$

and thus generate the $S U(N)$ [85]. $S U(N)$ as

Any Hermitian operator $\rho$ in $\mathscr{t}$ can be represented in terms of these generators of

$$
\rho=\frac{1}{N} I_{N}+\frac{1}{2} \sum_{j=1}^{N^{2}-1} r_{j} \lambda_{j}
$$

where $I_{N}$ is a unit matrix and $\mathbf{r}=\left(r_{1}, r_{2}, \ldots, r_{N^{2}-1}\right) \in \mathbb{R}^{N^{2}-1}$ and $\mathbf{r}$ is called Bloch vector. The set of all the Bloch vectors that constitute a density operator is known as the Bloch vector space $B\left(\mathbb{R}^{N^{2}-1}\right)$. 
A matrix of the form (2.13) is of unit trace and Hermitian, but it might not be positive. To guarantee the positivity restrictions must be imposed on the Bloch vector. It is shown that $B\left(\mathbb{R}^{N^{2}-1}\right)$ is a subset of the ball $D_{R}\left(\mathbb{R}^{N^{2}-1}\right)$ of radius $R=\sqrt{2(1-1 / N)}$, which is the minimum ball containing it, and that the ball $D_{r}\left(\mathbb{R}^{N^{2}-1}\right)$ of radius $r=\sqrt{2 / N(N-1)}$ is included in $B\left(\mathbb{R}^{N^{2}-1}\right)$ [86], that is,

$$
D_{r}\left(\mathbb{R}^{N^{2}-1}\right) \subseteq B\left(\mathbb{R}^{N^{2}-1}\right) \subseteq D_{R}\left(\mathbb{R}^{N^{2}-1}\right) .
$$

Let the dimensions of systems $A, B$, and $C$ be $d_{A}=N_{1}, d_{B}=N_{2}$, and $d_{C}=N_{3}$, respectively. Any tripartite quantum states $\rho_{A B C} \in \mathscr{l}_{A} \otimes \mathscr{l}_{B} \otimes \mathscr{l}_{C}$ can be written as

$$
\begin{aligned}
\rho_{A B C}= & I_{N_{1}} \otimes I_{N_{2}} \otimes M_{0}+\sum_{i=1}^{N_{1}^{2}-1} \lambda_{i}(1) \otimes I_{N_{2}} \otimes M_{i}+\sum_{j=1}^{N_{2}^{2}-1} I_{N_{1}} \otimes \lambda_{j}(2) \otimes \widetilde{M}_{j} \\
& +\sum_{i=1}^{N_{1}^{2}-1} \sum_{j=1}^{N_{2}^{2}-1} \lambda_{i}(1) \otimes \lambda_{j}(2) \otimes M_{i j},
\end{aligned}
$$

where $\lambda_{i}(1), \lambda_{j}(2)$ are the generators of $S U\left(N_{1}\right)$ and $S U\left(N_{2}\right) ; M_{i}, \widetilde{M}_{j}$, and $M_{i j}$ are operators of $\mathscr{t}_{C}$.

Theorem 2.2. Let $\mathbf{r} \in \mathbb{R}^{N_{1}^{2}-1}, \mathbf{s} \in \mathbb{R}^{N_{2}^{2}-1}$ and $|\mathbf{r}| \leq \sqrt{2 / N_{1}\left(N_{1}-1\right)},|\mathbf{s}| \leq \sqrt{2 / N_{2}\left(N_{2}-1\right)}$. For a tripartite quantum state $\rho \in \mathscr{l}_{A} \otimes \mathscr{l}_{B} \otimes \mathscr{l}_{C}$ with representation (2.15), one has [87]

$$
M_{0}-\sum_{i=1}^{N_{1}^{2}-1} r_{i} M_{i}-\sum_{j=1}^{N_{2}^{2}-1} s_{j} \widetilde{M}_{j}+\sum_{i=1}^{N_{1}^{2}-1} \sum_{j=1}^{N_{2}^{2}-1} r_{i} s_{j} M_{i j} \geq 0
$$

Proof. Since $\mathbf{r} \in \mathbb{R}^{N_{1}^{2}-1}, \mathbf{s} \in \mathbb{R}^{N_{2}^{2}-1}$ and $|\mathbf{r}| \leq \sqrt{2 / N_{1}\left(N_{1}-1\right)},|\mathbf{s}| \leq \sqrt{2 / N_{2}\left(N_{2}-1\right)}$, we have that $A_{1} \equiv(1 / 2)\left(\left(2 / N_{1}\right) I-\sum_{i=1}^{N_{1}^{2}-1} r_{i} \lambda_{i}(1)\right)$ and $A_{2} \equiv(1 / 2)\left(\left(2 / N_{2}\right) I-\sum_{j=1}^{N_{2}^{2}-1} s_{j} \lambda_{j}(2)\right)$ are positive Hermitian operators. Let $A=\sqrt{A_{1}} \otimes \sqrt{A_{2}} \otimes I_{N_{3}}$. Then $A \rho A \geq 0$ and $(A \rho A)^{\dagger}=A \rho A$. The partial trace of $A \rho A$ over $\mathscr{\ell}_{A}$ (and $\mathscr{L}_{B}$ ) should be also positive. Hence

$$
\begin{aligned}
0 \leq & \operatorname{Tr}_{A B}[A \rho A] \\
=\operatorname{Tr}_{A B}[ & {\left[A_{1} \otimes A_{2} \otimes M_{0}+\sum_{i} \sqrt{A_{1}} \lambda_{i}(1) \sqrt{A_{1}} \otimes A_{2} \otimes M_{i}\right.} \\
& \left.+\sum_{j} A_{1} \otimes \sqrt{A_{2}} \lambda_{j}(2) \sqrt{A_{2}} \otimes \widetilde{M}_{j}+\sum_{i j} \sqrt{A_{1}} \lambda_{i}(1) \sqrt{A_{1}} \otimes \sqrt{A_{2}} \lambda_{j}(2) \sqrt{A_{2}} \otimes M_{i j}\right] \\
=M_{0}- & \sum_{i=1}^{N_{1}^{2}-1} r_{i} M_{i}-\sum_{j=1}^{N_{2}^{2}-1} s_{j} \widetilde{M}_{j}+\sum_{i=1}^{N_{1}^{2}-1} \sum_{j=1}^{N_{2}^{2}-1} r_{i} s_{j} M_{i j} .
\end{aligned}
$$


Formula (2.16) is valid for any tripartite state. By setting $\mathbf{s}=0$ in (2.16), one can get a result for bipartite systems.

Corollary 2.3. Let $\rho_{A B} \in \mathscr{H}_{A} \otimes \mathscr{H}_{B}$, which can be generally written as $\rho_{A B}=I_{N_{1}} \otimes M_{0}+\sum_{j=1}^{N_{1}^{2}-1} \lambda_{j} \otimes$ $M_{j}$, then, for any $\mathbf{r} \in \mathbb{R}^{N_{1}^{2}-1}$ with $|\mathbf{r}| \leq \sqrt{2 / N_{1}\left(N_{1}-1\right)}, M_{0}-\sum_{j=1}^{N_{1}^{2}-1} r_{j} M_{j} \geq 0$.

A separable tripartite state $\rho_{A B C}$ can be written as

$$
\rho_{A B C}=\sum_{i} p_{i}\left|\psi_{i}^{A}\right\rangle\left\langle\psi_{i}^{A}|\otimes| \phi_{i}^{B}\right\rangle\left\langle\phi_{i}^{B}|\otimes| \omega_{i}^{C}\right\rangle\left\langle\omega_{i}^{C}\right|
$$

From (2.13) it can also be represented as

$$
\begin{aligned}
\rho_{A B C}= & \sum_{i} p_{i} \frac{1}{2}\left(\frac{2}{N_{1}} I_{N_{1}}+\sum_{k=1}^{N_{1}^{2}-1} a_{i}^{(k)} \lambda_{k}(1)\right) \otimes \frac{1}{2}\left(\frac{2}{N_{2}} I_{N_{2}}+\sum_{l=1}^{N_{2}^{2}-1} b_{i}^{(l)} \lambda_{l}(2)\right) \otimes\left|\omega_{i}^{C}\right\rangle\left\langle\omega_{i}^{C}\right| \\
= & I_{N_{1}} \otimes I_{N_{2}} \otimes \frac{1}{N_{1} N_{2}} \sum_{i} p_{i}\left|\omega_{i}^{C}\right\rangle\left\langle\omega_{i}^{C}\right| \\
& +\sum_{k=1}^{N_{1}^{2}-1} \lambda_{k}(1) \otimes I_{N_{2}} \otimes \frac{1}{2 N_{2}} \sum_{i} a_{i}^{(k)} p_{i}\left|\omega_{i}^{C}\right\rangle\left\langle\omega_{i}^{C}\right| \\
& +\sum_{l=1}^{N_{2}^{2}-1} I_{N_{1}} \otimes \lambda_{l}(2) \otimes \frac{1}{2 N_{1}} \sum_{i} b_{i}^{(l)} p_{i}\left|\omega_{i}^{C}\right\rangle\left\langle\omega_{i}^{C}\right| \\
& +\sum_{k}^{N_{1}^{2}-1} \sum_{l}^{N_{2}^{2}-1} \lambda_{k}(1) \otimes \lambda_{l}(2) \otimes \frac{1}{4} \sum_{i} a_{i}^{(k)} b_{i}^{(l)} p_{i}\left|\omega_{i}^{C}\right\rangle\left\langle\omega_{i}^{C}\right|
\end{aligned}
$$

where $\left(a_{i}^{(1)}, a_{i}^{(2)}, \ldots, a_{i}^{\left(N_{1}^{2}-1\right)}\right)$ and $\left(b_{i}^{(1)}, b_{i}^{(2)}, \ldots, b_{i}^{\left(N_{2}^{2}-1\right)}\right)$ are real vectors on the Bloch sphere satisfying $\left|\vec{a}_{i}\right|^{2}=\sum_{j=1}^{N_{1}^{2}-1}\left(a_{i}^{(j)}\right)^{2}=2\left(1-1 / N_{1}\right)$ and $\left|\overrightarrow{b_{i}}\right|^{2}=\sum_{j=1}^{N_{2}^{2}-1}\left(b_{i}^{(j)}\right)^{2}=2\left(1-1 / N_{2}\right)$.

Comparing (2.15) with (2.19), we have

$$
\begin{array}{ll}
M_{0}=\frac{1}{N_{1} N_{2}} \sum_{i} p_{i}\left|\omega_{i}^{C}\right\rangle\left\langle\omega_{i}^{C}\right|, & M_{k}=\frac{1}{2 N_{2}} \sum_{i} a_{i}^{(k)} p_{i}\left|\omega_{i}^{C}\right\rangle\left\langle\omega_{i}^{C}\right|, \\
\widetilde{M}_{l}=\frac{1}{2 N_{1}} \sum_{i} b_{i}^{(l)} p_{i}\left|\omega_{i}^{C}\right\rangle\left\langle\omega_{i}^{C}\right|, & M_{k l}=\frac{1}{4} \sum_{i} a_{i}^{(k)} b_{i}^{(l)} p_{i}\left|\omega_{i}^{C}\right\rangle\left\langle\omega_{i}^{C}\right| .
\end{array}
$$


For any $\left(N_{1}^{2}-1\right) \times\left(N_{1}^{2}-1\right)$ real matrix $R(1)$ and $\left(N_{2}^{2}-1\right) \times\left(N_{2}^{2}-1\right)$ real matrix $R(2)$ satisfying $\left(1 /\left(N_{1}-1\right)^{2}\right) I-R(1)^{T} R(1) \geq 0$ and $\left(1 /\left(N_{2}-1\right)^{2}\right) I-R(2)^{T} R(2) \geq 0$, we define a new matrix

$$
\mathcal{R}=\left(\begin{array}{ccc}
R(1) & 0 & 0 \\
0 & R(2) & 0 \\
0 & 0 & T
\end{array}\right)
$$

where $T$ is a transformation acting on an $\left(N_{1}^{2}-1\right) \times\left(N_{2}^{2}-1\right)$ matrix $M$ by

$$
T(M)=R(1) M R^{T}(2)
$$

Using $R$, we define a new operator $\gamma_{R}$ :

$$
\begin{aligned}
\gamma_{\mathcal{R}}\left(\rho_{A B C}\right)= & I_{N_{1}} \otimes I_{N_{2}} \otimes M_{0}^{\prime}+\sum_{i=1}^{N_{1}^{2}-1} \lambda_{i}(1) \otimes I_{N_{2}} \otimes M_{i}^{\prime}+\sum_{j=1}^{N_{2}^{2}-1} I_{N_{1}} \otimes \lambda_{j}(2) \otimes \widetilde{M}_{j}^{\prime} \\
& +\sum_{i=1}^{N_{1}^{2}-1} \sum_{j=1}^{N_{2}^{2}-1} \lambda_{i}(1) \otimes \lambda_{j}(2) \otimes M_{i j}^{\prime}
\end{aligned}
$$

where $M_{0}^{\prime}=M_{0}, M_{k}^{\prime}=\sum_{m=1}^{N_{1}^{2}-1} R_{k m}(1) M_{m}, \widetilde{M}_{l}^{\prime}=\sum_{n=1}^{N_{2}^{2}-1} R_{l n}(2) \widetilde{M}_{n}$, and $M_{i j}^{\prime}=(T(M))_{i j}=$ $\left(R(1) M R^{T}(2)\right)_{i j}$.

Theorem 2.4. If $\rho_{A B C}$ is separable, then [87] $\gamma_{R}\left(\rho_{A B C}\right) \geq 0$.

Proof. From (2.20) and (2.23) we get

$$
\begin{gathered}
M_{0}^{\prime}=M_{0}=\frac{1}{N_{1} N_{2}} \sum_{i} p_{i}\left|\omega_{i}^{C}\right\rangle\left\langle\omega_{i}^{C}\left|, \quad M_{k}^{\prime}=\frac{1}{2 N_{2}} \sum_{m i} R_{k m}(1) a_{i}^{(m)} p_{i}\right| \omega_{i}^{C}\right\rangle\left\langle\omega_{i}^{C}\right|, \\
\widetilde{M}_{l}^{\prime}=\frac{1}{2 N_{1}} \sum_{n i} R_{l n}(2) b_{i}^{(n)} p_{i}\left|\omega_{i}^{C}\right\rangle\left\langle\omega_{i}^{C}\left|, \quad M_{k l}^{\prime}=\frac{1}{4} \sum_{m n i} R_{k m}(1) a_{i}^{(m)} R_{l n}(2) b_{i}^{(n)} p_{i}\right| \omega_{i}^{C}\right\rangle\left\langle\omega_{i}^{C}\right| .
\end{gathered}
$$

A straightforward calculation gives rise to

$$
\begin{aligned}
\gamma_{\mathcal{R}}\left(\rho_{A B C}\right)=\sum_{i} p_{i} & \frac{1}{2}\left(\frac{2}{N_{1}} I_{N_{1}}+\sum_{k=1}^{N_{1}^{2}-1} \sum_{m=1}^{N_{1}^{2}-1} R_{k m}(1) a_{i}^{(m)} \lambda_{k}(1)\right) \\
\otimes & \frac{1}{2}\left(\frac{2}{N_{2}} I_{N_{2}}+\sum_{l=1}^{N_{2}^{2}-1} \sum_{n=1}^{N_{2}^{2}-1} R_{l n}(2) b_{i}^{(n)} \lambda_{l}(2)\right) \otimes\left|\omega_{i}^{C}\right\rangle\left\langle\omega_{i}^{C}\right| .
\end{aligned}
$$


As $\left(1 /\left(N_{1}-1\right)^{2}\right) I-R(1)^{T} R(1) \geq 0$ and $\left(1 /\left(N_{2}-1\right)^{2}\right) I-R(2)^{T} R(2) \geq 0$, we get

$$
\begin{gathered}
\left|\overrightarrow{a_{i}^{\prime}}\right|^{2}=\left|R(1) \overrightarrow{a_{i}}\right|^{2} \leq \frac{1}{\left(N_{1}-1\right)^{2}}\left|\overrightarrow{a_{i}}\right|^{2}=\frac{2}{N_{1}\left(N_{1}-1\right)}, \\
\left|\overrightarrow{b_{i}^{\prime}}\right|^{2}=\left|R(2) \overrightarrow{b_{i}}\right|^{2} \leq \frac{1}{\left(N_{2}-1\right)^{2}}\left|\overrightarrow{b_{i}}\right|^{2}=\frac{2}{N_{2}\left(N_{2}-1\right)} .
\end{gathered}
$$

Therefore $\gamma_{\mathcal{R}}\left(\rho_{A B C}\right)$ is still a density operator, that is, $\gamma_{\mathcal{R}}\left(\rho_{A B C}\right) \geq 0$.

Theorem 2.4 gives a necessary separability criterion for general tripartite systems. The result can be also applied to bipartite systems. Let $\rho_{A B} \in \mathscr{L}_{A} \otimes \mathscr{L}_{B}, \rho_{A B}=I_{N_{1}} \otimes M_{0}+\sum_{j=1}^{N_{1}^{2}-1} \lambda_{j} \otimes$ $M_{j}$. For any real $\left(N_{1}^{2}-1\right) \times\left(N_{1}^{2}-1\right)$ matrix $\mathcal{R}$ satisfying $\left(1 /\left(N_{1}-1\right)^{2}\right) I-\mathcal{R}^{T} \mathcal{R} \geq 0$ and any state $\rho_{A B}$, we define

$$
\gamma_{\mathcal{R}}\left(\rho_{A B}\right)=I_{N_{1}} \otimes M_{0}+\sum_{j=1}^{N_{1}^{2}-1} \lambda_{j} \otimes M_{j}^{\prime},
$$

where $M_{j}^{\prime}=\sum_{k} R_{j k} M_{k}$.

Corollary 2.5. For $\rho_{A B} \in \mathscr{H}_{A} \otimes \mathscr{H}_{B}$, if there exists an $\mathcal{R}$ with $\left(1 /\left(N_{1}-1\right)^{2}\right) I-\boldsymbol{R}^{T} \boldsymbol{R} \geq 0$ such that $\gamma_{\mathcal{R}}\left(\rho_{A B}\right)<0$, then $\rho_{A B}$ must be entangled.

For $2 \times N$ systems, the above corollary is reduced to the results in [88]. As an example we consider the $3 \times 3$ istropic states

$$
\rho_{I}=\frac{1-p}{9} I_{3} \otimes I_{3}+\frac{p}{3} \sum_{i, j=1}^{3}|i i\rangle\langle j j|=I_{3} \otimes\left(\frac{1}{9} I_{3}\right)+\sum_{i=1}^{5} \lambda_{i} \otimes\left(\frac{p}{6} \lambda_{i}\right)-\sum_{i=6}^{8} \lambda_{i} \otimes\left(\frac{p}{6} \lambda_{i}\right) .
$$

If we choose $R$ to be $\operatorname{Diag}\{1 / 2,1 / 2,1 / 2,1 / 2,1 / 2,-1 / 2,-1 / 2,-1 / 2\}$, we get that $\rho_{I}$ is entangled for $0.5<p \leq 1$.

For tripartite case, we take the following $3 \times 3 \times 3$ mixed state as an example:

$$
\rho=\frac{1-p}{27} I_{27}+p|\psi\rangle\langle\psi|
$$

where $|\psi\rangle=(1 / \sqrt{3})(|000\rangle+|111\rangle+|222\rangle)(\langle 000|+\langle 111|+\langle 222|)$. Taking $R(1)=R(2)=$ $\operatorname{Diag}\{1 / 2,1 / 2,1 / 2,1 / 2,1 / 2,-1 / 2,-1 / 2,-1 / 2\}$, we have that $\rho$ is entangled for $0.6248<p \leq$ 1 .

In fact the criterion for $2 \times N$ systems [88] is equivalent to the PPT criterion [89]. Similarly Theorem 2.4 is also equivalent to the PPT criterion for $2 \times 2 \times N$ systems. 


\subsubsection{Covariance Matrix Criterion}

In this subsection we study the separability problem by using the covariance matrix approach. We first give a brief review of covariance matrix criterion proposed in [49]. Let $\mathscr{H}_{d}^{A}$ and $\mathscr{H}_{d}^{B}$ be $d$-dimensional complex vector spaces and $\rho_{A B}$ a bipartite quantum state in $\mathscr{H}_{d}^{A} \otimes \mathscr{H}_{d}^{B}$. Let $A_{k}$ (resp., $B_{k}$ ) be $d^{2}$ observables on $\mathscr{H}_{d}^{A}\left(\right.$ resp., $\left.\mathscr{H}_{d}^{B}\right)$ such that they form an orthonormal normalized basis of the observable space, satisfying $\operatorname{Tr}\left[A_{k} A_{l}\right]=\delta_{k, l}$ (resp., $\left.\operatorname{Tr}\left[B_{k} B_{l}\right]=\delta_{k, l}\right)$. Consider the total set $\left\{M_{k}\right\}=\left\{A_{k} \otimes I, I \otimes B_{k}\right\}$. It can be proven that [44]

$$
\sum_{k=1}^{N^{2}}\left(M_{k}\right)^{2}=d I, \quad \sum_{k=1}^{N^{2}}\left\langle M_{k}\right\rangle^{2}=\operatorname{Tr}\left[\rho_{A B}^{2}\right] .
$$

The covariance matrix $\gamma$ is defined with entries

$$
\gamma_{i j}\left(\rho_{A B},\left\{M_{k}\right\}\right)=\frac{\left\langle M_{i} M_{j}\right\rangle+\left\langle M_{j} M_{i}\right\rangle}{2}-\left\langle M_{i}\right\rangle\left\langle M_{j}\right\rangle
$$

which has a block structure [49]

$$
r=\left(\begin{array}{ll}
A & C \\
C^{T} & B
\end{array}\right)
$$

where $A=\gamma\left(\rho_{A},\left\{A_{k}\right\}\right), B=\gamma\left(\rho_{B},\left\{B_{k}\right\}\right), C_{i j}=\left\langle A_{i} \otimes B_{j}\right\rangle_{\rho_{A B}}-\left\langle A_{i}\right\rangle_{\rho_{A}}\left\langle B_{j}\right\rangle_{\rho_{B}} \rho_{A}=\operatorname{Tr}_{B}\left[\rho_{A B}\right]$, and $\rho_{B}=\operatorname{Tr}_{A}\left[\rho_{A B}\right]$. Such covariance matrix has a concavity property: for a mixed density matrix $\rho=\sum_{k} p_{k} \rho_{k}$ with $p_{k} \geq 0$ and $\sum_{k} p_{k}=1$, one has $\gamma(\rho) \geq \sum_{k} p_{k} \gamma\left(\rho_{k}\right)$.

For a bipartite product state $\rho_{A B}=\rho_{A} \otimes \rho_{B}, C$ in (2.32) is zero. Generally if $\rho_{A B}$ is separable, then there exist states $\left|a_{k}\right\rangle\left\langle a_{k}\right|$ on $\mathscr{\ell}_{d}^{A},\left|b_{k}\right\rangle\left\langle b_{k}\right|$ on $\mathscr{\ell}_{d}^{B}$ and $p_{k}$ such that

$$
\gamma(\rho) \geq \kappa_{A} \oplus \kappa_{B}
$$

where $\kappa_{A}=\sum p_{k} \gamma\left(\left|a_{k}\right\rangle\left\langle a_{k}\right|,\left\{A_{k}\right\}\right), \kappa_{B}=\sum p_{k} \gamma\left(\left|b_{k}\right\rangle\left\langle b_{k}\right|,\left\{B_{k}\right\}\right)$.

For a separable bipartite state, it has been shown that [49]

$$
\sum_{i=1}^{d^{2}}\left|C_{i i}\right| \leq \frac{\left(1-\operatorname{Tr}\left[\rho_{A}^{2}\right]\right)+\left(1-\operatorname{Tr}\left[\rho_{B}^{2}\right]\right)}{2}
$$

Criterion (2.34) depends on the choice of the orthonormal normalized basis of the observables. In fact the term $\sum_{i=1}^{d^{2}}\left|C_{i i}\right|$ has an upper bound $\|C\|_{K F}$ which is invariant under unitary transformation and can be attained by choosing proper local orthonormal observable basis, where $\|C\|_{K F}$ stands for the Ky Fan norm of $C,\|C\|_{K F}=\operatorname{Tr}\left[\sqrt{C C^{\dagger}}\right]$, with + denoting the transpose and conjugation. It has been shown in [46] that if $\rho_{A B}$ is separable, then

$$
\|C\|_{K F} \leq \frac{\left(1-\operatorname{Tr}\left[\rho_{A}^{2}\right]\right)+\left(1-\operatorname{Tr}\left[\rho_{B}^{2}\right]\right)}{2}
$$


From the covariance matrix approach, we can also get an alternative criterion. From (2.32) and (2.33) we have that if $\rho_{A B}$ is separable, then

$$
X \equiv\left(\begin{array}{cc}
A-\kappa_{A} & C \\
C^{T} & B-\kappa_{B}
\end{array}\right) \geq 0 .
$$

Hence all the $2 \times 2$ minor submatrices of $X$ must be positive. Namely, one has

$$
\left|\begin{array}{cc}
\left(A-\kappa_{A}\right)_{i i} & C_{i j} \\
C_{j i} & \left(B-\kappa_{B}\right)_{j j}
\end{array}\right| \geq 0
$$

that is, $\left(A-\kappa_{A}\right)_{i i}\left(B-\kappa_{B}\right)_{j j} \geq C_{i j}^{2}$. Summing over all $i, j$ and using (2.30), we get

$$
\begin{aligned}
\sum_{i, j=1}^{d^{2}} C_{i, j}^{2} & \leq\left(\operatorname{Tr}[A]-\operatorname{Tr}\left[\kappa_{A}\right]\right)\left(\operatorname{Tr}[B]-\operatorname{Tr}\left[\kappa_{B}\right]\right) \\
& =\left(d-\operatorname{Tr}\left[\rho_{A}^{2}\right]-d+1\right)\left(d-\operatorname{Tr}\left[\rho_{B}^{2}\right]-d+1\right)=\left(1-\operatorname{Tr}\left[\rho_{A}^{2}\right]\right)\left(1-\operatorname{Tr}\left[\rho_{B}^{2}\right]\right)
\end{aligned}
$$

That is,

$$
\|C\|_{H S}^{2} \leq\left(1-\operatorname{Tr}\left[\rho_{A}^{2}\right]\right)\left(1-\operatorname{Tr}\left[\rho_{B}^{2}\right]\right)
$$

where $\|C\|_{H S}$ stands for the Euclid norm of $C$, that is, $\|C\|_{H S}=\sqrt{\operatorname{Tr}\left[C C^{\dagger}\right]}$.

Formulae (2.35) and (2.39) are independent and could be complement. When

$$
\sqrt{\left(1-\operatorname{Tr}\left[\rho_{A}^{2}\right]\right)\left(1-\operatorname{Tr}\left[\rho_{B}^{2}\right]\right)}<\|C\|_{H S} \leq\|C\|_{K F} \leq \frac{\left(1-\operatorname{Tr}\left[\rho_{A}^{2}\right]\right)+\left(1-\operatorname{Tr}\left[\rho_{B}^{2}\right]\right)}{2}
$$

(2.39) can recognize the entanglement but (2.35) cannot. When

$$
\|C\|_{H S} \leq \sqrt{\left(1-\operatorname{Tr}\left[\rho_{A}^{2}\right]\right)\left(1-\operatorname{Tr}\left[\rho_{B}^{2}\right]\right)} \leq \frac{\left(1-\operatorname{Tr}\left[\rho_{A}^{2}\right]\right)+\left(1-\operatorname{Tr}\left[\rho_{B}^{2}\right]\right)}{2}<\|C\|_{K F},
$$

(2.35) can recognize the entanglement while (2.39) cannot.

The separability criteria based on covariance matrix approach can be generalized to multipartite systems. We first consider the tripartite case $\rho_{A B C} \in \mathscr{H}_{d}^{A} \otimes \mathfrak{l}_{d}^{B} \otimes \mathfrak{l}_{d}^{C}$. Take $d^{2}$ observables $A_{k}$ on $\mathscr{l}_{A}$, respectively, $B_{k}$ on $\mathscr{l}_{B}$, respectively, $C_{k}$ on $\mathscr{L}_{C}$. Set $\left\{M_{k}\right\}=\left\{A_{k} \otimes I \otimes\right.$ $\left.I, I \otimes B_{k} \otimes I, I \otimes I \otimes C_{k}\right\}$. The covariance matrix defined by (2.31) has then the following block structure:

$$
\gamma=\left(\begin{array}{ccc}
A & D & E \\
D^{T} & B & F \\
E^{T} & F^{T} & C
\end{array}\right)
$$


where $A=\gamma\left(\rho_{A},\left\{A_{k}\right\}\right), B=\gamma\left(\rho_{B},\left\{B_{k}\right\}\right), C=\gamma\left(\rho_{C},\left\{C_{k}\right\}\right), D_{i j}=\left\langle A_{i} \otimes B_{j}\right\rangle_{\rho_{A B}}-\left\langle A_{i}\right\rangle_{\rho_{A}}\left\langle B_{j}\right\rangle_{\rho_{B}}$, $E_{i j}=\left\langle A_{i} \otimes C_{j}\right\rangle_{\rho_{A C}}-\left\langle A_{i}\right\rangle_{\rho_{A}}\left\langle C_{j}\right\rangle_{\rho_{C}}$ and $F_{i j}=\left\langle B_{i} \otimes C_{j}\right\rangle_{\rho_{B C}}-\left\langle B_{i}\right\rangle_{\rho_{B}}\left\langle C_{j}\right\rangle_{\rho_{C}}$.

Theorem 2.6. If $\rho_{A B C}$ is fully separable, then [90]

$$
\begin{gathered}
\|D\|_{H S}^{2} \leq\left(1-\operatorname{Tr}\left[\rho_{A}^{2}\right]\right)\left(1-\operatorname{Tr}\left[\rho_{B}^{2}\right]\right), \\
\|E\|_{H S}^{2} \leq\left(1-\operatorname{Tr}\left[\rho_{A}^{2}\right]\right)\left(1-\operatorname{Tr}\left[\rho_{C}^{2}\right]\right), \\
\|F\|_{H S}^{2} \leq\left(1-\operatorname{Tr}\left[\rho_{B}^{2}\right]\right)\left(1-\operatorname{Tr}\left[\rho_{C}^{2}\right]\right), \\
2\|D\|_{K F} \leq\left(1-\operatorname{Tr}\left[\rho_{A}^{2}\right]\right)+\left(1-\operatorname{Tr}\left[\rho_{B}^{2}\right]\right), \\
2\|E\|_{K F} \leq\left(1-\operatorname{Tr}\left[\rho_{A}^{2}\right]\right)+\left(1-\operatorname{Tr}\left[\rho_{C}^{2}\right]\right), \\
2\|F\|_{K F} \leq\left(1-\operatorname{Tr}\left[\rho_{B}^{2}\right]\right)+\left(1-\operatorname{Tr}\left[\rho_{C}^{2}\right]\right) .
\end{gathered}
$$

Proof. For a tripartite product state $\rho_{A B C}=\rho_{A} \otimes \rho_{B} \otimes \rho_{C}, D, E$, and $F$ in (2.42) are zero. If $\rho_{A B C}$ is fully separable, then there exist states $\left|a_{k}\right\rangle\left\langle a_{k}\right|$ in $\mathscr{H}_{d}^{A},\left|b_{k}\right\rangle\left\langle b_{k}\right|$ in $\mathscr{H}_{d}^{B}$, and $\left|c_{k}\right\rangle\left\langle c_{k}\right|$ in $\mathscr{H}_{d}^{C}$, and $p_{k}$ such that $\gamma(\rho) \geq \kappa_{A} \oplus \kappa_{B} \oplus \kappa_{C}$, where $\kappa_{A}=\sum p_{k} \gamma\left(\left|a_{k}\right\rangle\left\langle a_{k}\right|,\left\{A_{k}\right\}\right), \kappa_{B}=$ $\sum p_{k} \gamma\left(\left|b_{k}\right\rangle\left\langle b_{k}\right|,\left\{B_{k}\right\}\right)$, and $\kappa_{C}=\sum p_{k} \gamma\left(\left|c_{k}\right\rangle\left\langle c_{k}\right|,\left\{C_{k}\right\}\right)$, that is,

$$
Y \equiv\left(\begin{array}{ccc}
A-\kappa_{A} & D & E \\
D^{T} & B-\kappa_{B} & F \\
E^{T} & F^{T} & C-\kappa_{C}
\end{array}\right) \geq 0
$$

Thus all the $2 \times 2$ minor submatrices of $Y$ must be positive. Selecting one with two rows and columns from the first two block rows and columns of $Y$, we have

$$
\left|\begin{array}{cc}
\left(A-\kappa_{A}\right)_{i i} & D_{i j} \\
D_{j i} & \left(B-\kappa_{B}\right)_{j j}
\end{array}\right| \geq 0
$$

that is, $\left(A-\kappa_{A}\right)_{i i}\left(B-\kappa_{B}\right)_{j j} \geq\left|D_{i j}\right|^{2}$. Summing over all $i, j$ and using (2.30), we get

$$
\begin{aligned}
\|D\|_{H S}^{2} & =\sum_{i, j=1}^{d^{2}} D_{i, j}^{2} \leq\left(\operatorname{Tr}[A]-\operatorname{Tr}\left[\kappa_{A}\right]\right)\left(\operatorname{Tr}[B]-\operatorname{Tr}\left[\kappa_{B}\right]\right. \\
& =\left(d-\operatorname{Tr}\left[\rho_{A}^{2}\right]-d+1\right)\left(d-\operatorname{Tr}\left[\rho_{B}^{2}\right]-d+1\right)=\left(1-\operatorname{Tr}\left[\rho_{A}^{2}\right]\right)\left(1-\operatorname{Tr}\left[\rho_{B}^{2}\right]\right),
\end{aligned}
$$

which proves (2.43). Equations (2.44) and (2.45) can be similarly proved. 
From (2.50) we also have $\left(A-\kappa_{A}\right)_{i i}+\left(B-\kappa_{B}\right)_{i i} \geq 2\left|D_{i i}\right|$. Therefore

$$
\begin{aligned}
\sum_{i}\left|D_{i i}\right| & \leq \frac{\left(\operatorname{Tr}[A]-\operatorname{Tr}\left[\mathcal{\kappa}_{A}\right]\right)+\left(\operatorname{Tr}[B]-\operatorname{Tr}\left[\kappa_{B}\right]\right.}{2} \\
& =\frac{\left(d-\operatorname{Tr}\left[\rho_{A}^{2}\right]-d+1\right)+\left(d-\operatorname{Tr}\left[\rho_{B}^{2}\right]-d+1\right)}{2} \\
& =\frac{\left(1-\operatorname{Tr}\left[\rho_{A}^{2}\right]\right)+\left(1-\operatorname{Tr}\left[\rho_{B}^{2}\right]\right)}{2} .
\end{aligned}
$$

Note that $\sum_{i=1}^{d^{2}} D_{i i} \leq \sum_{i=1}^{d^{2}}\left|D_{i i}\right|$. By using that $\operatorname{Tr}[M U] \leq\|M\|_{K F}=\operatorname{Tr}\left[\sqrt{M M^{\dagger}}\right]$ for any matrix $M$ and any unitary $U[91]$, we have $\sum_{i=1}^{d^{2}} D_{i i} \leq\|D\|_{K F}$.

Let $D=U^{\dagger} \Lambda V$ be the singular value decomposition of $D$. Make a transformation of the orthonormal normalized basis of the local orthonormal observable space $\widetilde{A}_{i}=\sum_{l} U_{i l} A_{l}$ and $\widetilde{B}_{j}=\sum_{m} V_{j m}^{*} B_{m}$. In the new basis we have

$$
\tilde{D}_{i j}=\sum_{l m} U_{i l} D_{l m} V_{j m}=\left(U D V^{\dagger}\right)_{i j}=\Lambda_{i j}
$$

Then (2.52) becomes

$$
\sum_{i=1}^{d^{2}} \widetilde{D}_{i i}=\|D\|_{K F} \leq \frac{\left(1-\operatorname{Tr}\left[\rho_{A}^{2}\right]\right)+\left(1-\operatorname{Tr}\left[\rho_{B}^{2}\right]\right)}{2}
$$

which proves (2.46). Equations (2.47) and (2.48) can be similarly treated.

We consider now the case that $\rho_{A B C}$ is bipartite separable.

Theorem 2.7. If $\rho_{A B C}$ is a bipartite separable state with respect to the bipartite partition of the subsystems $A$ and $B C$ (resp., $A B$ and C; resp., $A C$ and B), then (2.43), (2.44) and (2.46), (2.47) (resp., (2.44), (2.45) and (2.47), (2.48); resp., (2.43), (2.45) and (2.46), (2.48)) must hold [90].

Proof. We prove the case that $\rho_{A B C}$ is bipartite separable with respect to the $A$ system and $B C$ systems partition. The other cases can be similarly treated. In this case the matrices $D$ and $E$ in the covariance matrix (2.42) are zero. $\rho_{A B C}$ takes the form $\rho_{A B C}=\sum_{m} p_{m} \rho_{A}^{m} \otimes \rho_{B C}^{m}$. Define $\kappa_{A}=\sum p_{m} \gamma\left(\rho_{A}^{m},\left\{A_{k}\right\}\right), \kappa_{B C}=\sum p_{m} \gamma\left(\rho_{B C}^{m},\left\{B_{k} \otimes I, I \otimes C_{k}\right\}\right) . \kappa_{B C}$ has a form

$$
\kappa_{B C}=\left(\begin{array}{cc}
\kappa_{B} & F^{\prime} \\
\left(F^{\prime}\right)^{T} & \kappa_{C}
\end{array}\right)
$$


where $\kappa_{B}=\sum p_{k} \gamma\left(\left|b_{k}\right\rangle\left\langle b_{k}\right|,\left\{B_{k}\right\}\right)$ and $\kappa_{C}=\sum p_{k} \gamma\left(\left|c_{k}\right\rangle\left\langle c_{k}\right|,\left\{C_{k}\right\}\right),\left(F^{\prime}\right)_{i j}=\sum_{m} p_{m}\left(\left\langle B_{i} \otimes\right.\right.$ $\left.\left.C_{j}\right\rangle_{\rho_{B C}^{m}}-\left\langle B_{i}\right\rangle_{\rho_{B}^{m}}\left\langle C_{j}\right\rangle_{\rho_{C}^{m}}\right)$. By using the concavity of covariance matrix we have

$$
\gamma\left(\rho_{A B C}\right) \geq \sum_{m} p_{m} \gamma\left(\rho_{A}^{m} \otimes \rho_{B C}^{m}\right)=\left(\begin{array}{ccc}
\kappa_{A} & 0 & 0 \\
0 & \kappa_{B} & F^{\prime} \\
0 & \left(F^{\prime}\right)^{T} & \kappa_{C}
\end{array}\right) .
$$

Accounting to the method used in proving Theorem 2, we get (2.43), (2.44), and (2.46), (2.47).

From Theorems 2.6 and 2.7 we have the following corollary.

Corollary 2.8. If two of the inequalities (2.43), (2.44), and (2.45) (or (2.46), (2.47), and (2.48)) are violated, then the state must be fully entangled.

The result of Theorem 2.6 can be generalized to general multipartite case $\rho \in \mathscr{L}_{d}^{(1)} \otimes$ $\mathscr{H}_{d}^{(2)} \otimes \cdots \otimes \mathscr{H}_{d}^{(N)}$. Define $\widehat{A}_{\alpha}^{i}=I \otimes I \otimes \cdots \lambda_{\alpha} \otimes I \otimes \cdots \otimes I$, where $\lambda_{0}=I / d, \lambda_{\alpha}\left(\alpha=1,2, \ldots, d^{2}-1\right)$ are the normalized generators of $S U(d)$ satisfying $\operatorname{Tr}\left[\lambda_{\alpha} \lambda_{\beta}\right]=\delta_{\alpha \beta}$ and acting on the $i$ th system $\mathscr{H}_{d}^{(i)}, i=1,2, \ldots, N$. Denote $\left\{M_{k}\right\}$ as the set of all $\widehat{A}_{\alpha}^{i}$. Then the covariance matrix of $\rho$ can be written as

$$
r(\rho)=\left(\begin{array}{ccc}
A_{11} & A_{12} \cdots & A_{1 N} \\
A_{12}^{T} & A_{22} \cdots & A_{2 N} \\
\vdots & \vdots & \vdots \\
A_{1 N}^{T} & A_{2 N}^{T} \cdots & A_{N N}
\end{array}\right)
$$

where $\mathcal{A}_{i i}=\gamma\left(\rho,\left\{\widehat{A}_{k}^{i}\right\}\right)$ and $\left(\mathscr{A}_{i j}\right)_{m n}=\left\langle\widehat{A}_{m}^{i} \otimes \widehat{A}_{n}^{j}\right\rangle-\left\langle\widehat{A}_{m}^{i}\right\rangle\left\langle\widehat{A}_{n}^{j}\right\rangle$ for $i \neq j$.

For a product state $\rho_{12 \cdots N}, \mathcal{A}_{i j}, i \neq j$, in (2.57) are zero matrices. Define

$$
\kappa_{\mathcal{A}_{i i}}=\sum_{k} p_{k} \gamma\left(\left|\psi_{k}^{i}\right\rangle\left\langle\psi_{k}^{i}\right|,\left\{\widehat{A}_{l}^{i}\right\}\right)
$$

Then for a fully separable multipartite state $\rho=\sum_{k} p_{k}\left|\psi_{k}^{1}\right\rangle\left\langle\psi_{k}^{1}|\otimes| \psi_{k}^{2}\right\rangle\left\langle\psi_{k}^{2}|\otimes \cdots \otimes| \psi_{k}^{N}\right\rangle\left\langle\psi_{k}^{N}\right|$, one has

$$
Z=\left(\begin{array}{ccc}
\mathcal{A}_{11}-\kappa_{A_{11}} & \mathcal{A}_{12} \cdots & \mathcal{A}_{1 N} \\
\mathcal{A}_{12}^{T} & \mathcal{A}_{22}-\kappa_{A_{22}} \cdots & \mathcal{A}_{2 N} \\
\vdots & \vdots & \vdots \\
\mathcal{A}_{1 N}^{T} & \mathcal{A}_{2 N}^{T} \cdots & \mathcal{A}_{N N}-\kappa_{A_{N N}}
\end{array}\right) \geq 0
$$

from which we have the following separability criterion for multipartite systems. 
Theorem 2.9. If a state $\rho \in \mathscr{H}_{d}^{(1)} \otimes \mathscr{H}_{d}^{(2)} \otimes \cdots \otimes \mathscr{H}_{d}^{(N)}$ is fully separable, then the following inequalities

$$
\begin{aligned}
\left\|A_{i j}\right\|_{H S}^{2} & \leq\left(1-\operatorname{Tr}\left[\rho_{i}^{2}\right]\right)\left(1-\operatorname{Tr}\left[\rho_{j}^{2}\right]\right), \\
\left\|A_{i j}\right\|_{K F} & \leq \frac{\left(1-\operatorname{Tr}\left[\rho_{i}^{2}\right]\right)+\left(1-\operatorname{Tr}\left[\rho_{j}^{2}\right]\right)}{2}
\end{aligned}
$$

must be fulfilled for any $i \neq j[90]$.

\subsection{Normal Form of Quantum States}

In this subsection we show that the correlation matrix $(\mathrm{CM})$ criterion can be improved from the normal form obtained under filtering transformations. Based on CM criterion entanglement witness in terms of local orthogonal observables (LOOs) [92] for both bipartite and multipartite systems can be also constructed.

For bipartite case, $\rho \in \mathscr{\ell}=\mathscr{\ell}_{A} \otimes \mathscr{\ell}_{B}$ with $\operatorname{dim} \mathscr{\ell}_{A}=M$, $\operatorname{dim} \mathfrak{l}_{B}=N$, and $M \leq N$ is mapped to the following form under local filtering transformations [93]:

$$
\rho \longrightarrow \tilde{\rho}=\frac{\left(F_{A} \otimes F_{B}\right) \rho\left(F_{A} \otimes F_{B}\right)^{\dagger}}{\operatorname{Tr}\left[\left(F_{A} \otimes F_{B}\right) \rho\left(F_{A} \otimes F_{B}\right)^{\dagger}\right]},
$$

where $F_{A / B} \in G L(M / N, \mathbb{C})$ are arbitrary invertible matrices. This transformation is also known as stochastic local operations assisted by classical communication (SLOCC). By the definition it is obvious that filtering transformation will preserve the separability of a quantum state.

It has been shown that under local filtering operations one can transform a strictly positive $\rho$ into a normal form [94]:

$$
\tilde{\rho}=\frac{\left(F_{A} \otimes F_{B}\right) \rho\left(F_{A} \otimes F_{B}\right)^{\dagger}}{\operatorname{Tr}\left[\left(F_{A} \otimes F_{B}\right) \rho\left(F_{A} \otimes F_{B}\right)^{\dagger}\right]}=\frac{1}{M N}\left(I+\sum_{i=1}^{M^{2}-1} \xi_{i} G_{i}^{A} \otimes G_{i}^{B}\right),
$$

where $\xi_{i} \geq 0$ and $G_{i}^{A}$ and $G_{i}^{B}$ are some traceless orthogonal observables. The matrices $F_{A}$ and $F_{B}$ can be obtained by minimizing the function

$$
f(A, B)=\frac{\operatorname{Tr}[\rho(A \otimes B)]}{(\operatorname{det} A)^{1 / M}(\operatorname{det} B)^{1 / N}}
$$

where $A=F_{A}^{\dagger} F_{A}$ and $B=F_{B}^{\dagger} F_{B}$. In fact, one can choose $F_{A}^{0} \equiv\left|\operatorname{det}\left(\rho_{A}\right)\right|^{1 / 2 M}\left(\sqrt{\rho_{A}}\right)^{-1}$, and $F_{B}^{0} \equiv\left|\operatorname{det}\left(\rho_{B}^{\prime}\right)\right|^{1 / 2 N}\left(\sqrt{\rho_{B}^{\prime}}\right)^{-1}$, where $\rho_{B}^{\prime}=\operatorname{Tr}_{A}\left[I \otimes\left(\sqrt{\rho_{A}}\right)^{-1} \rho I \otimes\left(\sqrt{\rho_{A}}\right)^{-1}\right]$. Then by iterations one can get the optimal $A$ and $B$. In particular, there is a matlab code available in [95]. 
For bipartite separable states $\rho$, the CM separability criterion [96] says that

$$
\|T\|_{K F} \leq \sqrt{M N(M-1)(N-1)},
$$

where $T$ is an $\left(M^{2}-1\right) \times\left(N^{2}-1\right)$ matrix with $T_{i j}=M N \cdot \operatorname{Tr}\left[\rho \lambda_{i}^{A} \otimes \lambda_{j}^{B}\right],\|T\|_{K F}$ stands for the trace norm of $T, \lambda_{k}^{A / B}$ S are the generators of $S U(M / N)$ and have been chosen to be normalized, and $\operatorname{Tr}\left[\lambda_{k}^{(A / B)} \lambda_{l}^{(A / B)}\right]=\delta_{k l}$.

As the filtering transformation does not change the separability of a state, one can study the separability of $\tilde{\rho}$ instead of $\rho$. Under the normal form (2.62) the criterion (2.64) becomes

$$
\sum_{i} \xi_{i} \leq \sqrt{M N(M-1)(N-1)}
$$

In [44] a separability criterion based on local uncertainty relation (LUR) has been obtained. It says that, for any separable state $\rho$,

$$
1-\sum_{k}\left\langle G_{k}^{A} \otimes G_{k}^{B}\right\rangle-\frac{1}{2}\left\langle G_{k}^{A} \otimes I-I \otimes G_{k}^{B}\right\rangle^{2} \geq 0
$$

where $G_{k}^{A / B}$ s are LOOs such as the normalized generators of $S U(M / N)$ and $G_{k}^{A}=0$ for $k=M^{2}+1, \ldots, N^{2}$. The criterion is shown to be strictly stronger than the realignment criterion [61]. Under the normal form (2.62) criterion (2.66) becomes

$$
1-\sum_{k}\left\langle G_{k}^{A} \otimes G_{k}^{B}\right\rangle-\frac{1}{2}\left\langle G_{k}^{A} \otimes I-I \otimes G_{k}^{B}\right\rangle^{2}=1-\frac{1}{M N} \sum_{k} \xi_{k}-\frac{1}{2}\left(\frac{1}{M}+\frac{1}{N}\right) \geq 0,
$$

that is,

$$
\sum_{k} \xi_{k} \leq M N-\frac{M+N}{2}
$$

As $\sqrt{M N(M-1)(N-1)} \leq M N-(M+N) / 2$ holds for any $M$ and $N$, from (2.65) and (2.68) it is obvious that the CM criterion recognizes entanglement better when the normal form is taken into account. 
We now consider multipartite systems. Let $\rho$ be a strictly positive density matrix in $\mathscr{H}=\mathscr{H}_{1} \otimes \mathscr{H}_{2} \otimes \cdots \otimes \mathscr{H}_{N}$ and $\operatorname{dim} \mathscr{H}_{i}=d_{i} . \rho$ can be generally expressed in terms of the $\operatorname{SU}(n)$ generators $\lambda_{\alpha_{k}}[97]$ as

$$
\begin{aligned}
& \rho=\frac{1}{\Pi_{i}^{N} d_{i}}\left(\otimes_{j}^{N} I_{d_{j}}+\sum_{\left\{\mu_{1}\right\}} \sum_{\alpha_{1}} \tau_{\alpha_{1}}^{\left\{\mu_{1}\right\}} \lambda_{\alpha_{1}}^{\left\{\mu_{1}\right\}}+\sum_{\left\{\mu_{1} \mu_{2}\right\} \alpha_{1} \alpha_{2}} \tau_{\alpha_{1} \alpha_{2}}^{\left\{\mu_{1} \mu_{2}\right\}} \lambda_{\alpha_{1}}^{\left\{\mu_{1}\right\}} \lambda_{\alpha_{2}}^{\left\{\mu_{2}\right\}}\right. \\
&+\sum_{\left\{\mu_{1} \mu_{2} \mu_{3}\right\}} \sum_{\alpha_{1} \alpha_{2} \alpha_{3}} \tau_{\alpha_{1} \alpha_{2} \alpha_{3}}^{\left\{\mu_{1} \mu_{2} \mu_{3}\right\}} \lambda_{\alpha_{1}}^{\left\{\mu_{1}\right\}} \lambda_{\alpha_{2}}^{\left\{\mu_{2}\right\}} \lambda_{\alpha_{3}}^{\left\{\mu_{3}\right\}} \\
&+\cdots+\sum_{\left\{\mu_{1} \mu_{2} \cdots \mu_{M}\right\}} \sum_{\alpha_{1} \alpha_{2} \cdots \alpha_{M}} \tau_{\alpha_{1} \alpha_{2} \cdots \alpha_{M}}^{\left\{\mu_{1} \mu_{2} \cdots \mu_{M}\right\}} \lambda_{\alpha_{1}}^{\left\{\mu_{1}\right\}} \lambda_{\alpha_{2}}^{\left\{\mu_{2}\right\}} \cdots \lambda_{\alpha_{M}}^{\left\{\mu_{M}\right\}} \\
&\left.+\cdots+\sum_{\alpha_{1} \alpha_{2} \cdots \alpha_{N}} \tau_{\alpha_{1} \alpha_{2} \cdots \alpha_{M}}^{\{1,2, \ldots, N\}} \lambda_{\alpha_{1}}^{\{1\}} \lambda_{\alpha_{2}}^{\{2\}} \cdots \lambda_{\alpha_{N}}^{\{N\}}\right)
\end{aligned}
$$

where $\lambda_{\alpha_{k}}^{\left\{\mu_{k}\right\}}=I_{d_{1}} \otimes I_{d_{2}} \otimes \cdots \otimes \lambda_{\alpha_{k}} \otimes I_{d_{\mu_{k}+1}} \otimes \cdots \otimes I_{d_{N}}$ with $\lambda_{\alpha_{k}}$ appears at the $\mu_{k}$ th position and

$$
\tau_{\alpha_{1} \alpha_{2} \cdots \alpha_{M}}^{\left\{\mu_{1} \mu_{2} \cdots \mu_{M}\right\}}=\frac{\prod_{i=1}^{M} d_{\mu_{i}}}{2^{M}} \operatorname{Tr}\left[\rho \lambda_{\alpha_{1}}^{\left\{\mu_{1}\right\}} \lambda_{\alpha_{2}}^{\left\{\mu_{2}\right\}} \cdots \lambda_{\alpha_{M}}^{\left\{\mu_{M}\right\}}\right]
$$

The generalized CM criterion says that if $\rho$ in (2.69) is fully separable, then

$$
\left\|\tau^{\left\{\mu_{1}, \mu_{2}, \ldots, \mu_{M}\right\}}\right\|_{K F} \leq \sqrt{\frac{1}{2^{M}} \prod_{k=1}^{M} d_{\mu_{k}}\left(d_{\mu_{k}}-1\right)},
$$

for $2 \leq M \leq N,\left\{\mu_{1}, \mu_{2}, \ldots, \mu_{M}\right\} \subset\{1,2, \ldots, N\}$. The KF norm is defined by

$$
\left\|\tau^{\left\{\mu_{1}, \mu_{2}, \ldots, \mu_{M}\right\}}\right\|_{K F}=\max _{m=1,2, \ldots, M}\left\|\tau_{(m)}\right\|_{K F^{\prime}}
$$

where $\tau_{(m)}$ is a kind of matrix unfolding of $\tau\left\{\mu_{1}, \mu_{2}, \ldots, \mu_{M}\right\}$.

The criterion (2.71) can be improved by investigating the normal form of (2.69).

Theorem 2.10. By filtering transformations of the form

$$
\tilde{\rho}=F_{1} \otimes F_{2} \otimes \cdots \otimes F_{N} \rho F_{1}^{\dagger} \otimes F_{2}^{\dagger} \otimes F_{N^{\prime}}^{\dagger}
$$


where $F_{i} \in G L\left(d_{i}, \mathbb{C}\right), i=1,2, \ldots N$, followed by normalization, any strictly positive state $\rho$ can be transformed into a normal form [98]:

$$
\begin{aligned}
& \rho=\frac{1}{\Pi_{i}^{N} d_{i}}\left(\otimes_{j}^{N} I_{d_{j}}+\sum_{\left\{\mu_{1} \mu_{2}\right\}} \sum_{\alpha_{1} \alpha_{2}} \tau_{\alpha_{1} \alpha_{2}}^{\left\{\mu_{1} \mu_{2}\right\}} \lambda_{\alpha_{1}}^{\left\{\mu_{1}\right\}} \lambda_{\alpha_{2}}^{\left\{\mu_{2}\right\}}\right. \\
&+\sum_{\left\{\mu_{1} \mu_{2} \mu_{3}\right\}} \sum_{\alpha_{1} \alpha_{2} \alpha_{3}} \tau_{\alpha_{1} \alpha_{2} \alpha_{3}}^{\left\{\mu_{1} \mu_{2} \mu_{3}\right\}} \lambda_{\alpha_{1}}^{\left\{\mu_{1}\right\}} \lambda_{\alpha_{2}}^{\left\{\mu_{2}\right\}} \lambda_{\alpha_{3}}^{\left\{\mu_{3}\right\}} \\
&+\cdots+\sum_{\left\{\mu_{1} \mu_{2} \cdots \mu_{M}\right\}} \sum_{\alpha_{1} \alpha_{2} \cdots \alpha_{M}} \tau_{\alpha_{1} \alpha_{2} \cdots \alpha_{M}}^{\left\{\mu_{1} \mu_{2} \cdots \mu_{M}\right\}} \lambda_{\alpha_{1}}^{\left\{\mu_{1}\right\}} \lambda_{\alpha_{2}}^{\left\{\mu_{2}\right\}} \cdots \lambda_{\alpha_{M}}^{\left\{\mu_{M}\right\}} \\
&\left.+\cdots+\sum_{\alpha_{1} \alpha_{2} \cdots \alpha_{N}} \tau_{\alpha_{1} \alpha_{2} \cdots \alpha_{M}}^{\{1,2, \ldots N\}} \lambda_{\alpha_{1}}^{\{1\}} \lambda_{\alpha_{2}}^{\{2\}} \cdots \lambda_{\alpha_{N}}^{\{N\}}\right) .
\end{aligned}
$$

Proof. Let $D_{1}, D_{2}, \ldots, D_{N}$ be the sets of density matrices of the $N$ subsystems. The cartesian product $D_{1} \times D_{2} \times \cdots \times D_{N}$ consisting of all product density matrices $\rho_{1} \otimes \rho_{2} \otimes \cdots \otimes \rho_{N}$ with normalization $\operatorname{Tr}\left[\rho_{i}\right]=1, i=1,2, \ldots, N$, is a compact set of matrices on the full Hilbert space $\mathscr{H}$. For the given density matrix $\rho$ we define the following function of $\rho_{i}$ :

$$
f\left(\rho_{1}, \rho_{2}, \ldots, \rho_{N}\right)=\frac{\operatorname{Tr}\left[\rho\left(\rho_{1} \otimes \rho_{2} \otimes \cdots \otimes \rho_{N}\right)\right]}{\prod_{i=1}^{N} \operatorname{det}\left(\rho_{i}\right)^{1 / d_{i}}} .
$$

The function is well defined on the interior of $D_{1} \times D_{2} \times \cdots \times D_{N}$ where $\operatorname{det} \rho_{i}>0$. As $\rho$ is assumed to be strictly positive, we have $\operatorname{Tr}\left[\rho\left(\rho_{1} \otimes \rho_{2} \otimes \cdots \otimes \rho_{N}\right)\right]>0$. Since $D_{1} \times D_{2} \times \cdots \times D_{N}$ is compact, we have $\operatorname{Tr}\left[\rho\left(\rho_{1} \otimes \rho_{2} \otimes \cdots \otimes \rho_{N}\right)\right] \geq C>0$ with a lower bound $C$ depending on $\rho$.

It follows that $f \rightarrow \infty$ on the boundary of $D_{1} \times D_{2} \times \cdots \times D_{N}$ where at least one of the $\rho_{i}$ s satisfies $\operatorname{det} \rho_{i}=0$. It follows further that $f$ has a positive minimum on the interior of $D_{1} \times D_{2} \times \cdots \times D_{N}$ with the minimum value attained for at least one product density matrix $\tau_{1} \otimes \tau_{2} \otimes \cdots \otimes \tau_{N}$ with $\operatorname{det} \tau_{i}>0, i=1,2, \ldots, N$. Any positive density matrix $\tau_{i}$ with det $\tau_{i}>0$ can be factorized in terms of Hermitian matrices $F_{i}$ as

$$
\tau_{i}=F_{i}^{\dagger} F_{i}
$$

where $F_{i} \in G L\left(d_{i}, \mathbb{C}\right)$. Denote that $F=F_{1} \otimes F_{2} \otimes \cdots \otimes F_{N}$, so that $\tau_{1} \otimes \tau_{2} \otimes \cdots \otimes \tau_{N}=F^{\dagger} F$. Set $\tilde{\rho}=F \rho F^{\dagger}$ and define

$$
\begin{aligned}
\tilde{f}\left(\rho_{1}, \rho_{2}, \ldots, \rho_{N}\right) & =\frac{\operatorname{Tr}\left[\tilde{\rho}\left(\rho_{1} \otimes \rho_{2} \otimes \cdots \otimes \rho_{N}\right)\right]}{\prod_{i=1}^{N} \operatorname{det}\left(\rho_{i}\right)^{1 / d_{i}}} \\
& =\prod_{i=1}^{N} \operatorname{det}\left(\tau_{i}\right)^{1 / d_{i}} \cdot \frac{\operatorname{Tr}\left[\rho\left(F_{1}^{\dagger} \rho_{1} F_{1} \otimes F_{2}^{\dagger} \rho_{2} F_{2} \otimes \cdots \otimes F_{N}^{\dagger} \rho_{N} F_{N}\right)\right]}{\prod_{i=1}^{N} \operatorname{det}\left(\tau_{i}\right)^{1 / d_{i}} \operatorname{det}\left(\rho_{i}\right)^{1 / d_{i}}} \\
& =\prod_{i=1}^{N} \operatorname{det}\left(\tau_{i}\right)^{1 / d_{i}} \cdot f\left(F_{1}^{\dagger} \rho_{1} F_{1}, F_{2}^{\dagger} \rho_{2} F_{2}, \ldots, F_{N}^{\dagger} \rho_{N} F_{N}\right) .
\end{aligned}
$$


We see that, when $F_{i}^{\dagger} \rho_{i} F_{i}=\tau_{i}, \tilde{f}$ has a minimum and

$$
\rho_{1} \otimes \rho_{2} \otimes \cdots \otimes \rho_{N}=\left(F^{\dagger}\right)^{-1} \tau_{1} \otimes \tau_{2} \otimes \cdots \otimes \tau_{N} F^{-1}=I .
$$

Since $\tilde{f}$ is stationary under infinitesimal variations about the minimum, it follows that

$$
\operatorname{Tr}\left[\tilde{\rho} \delta\left(\rho_{1} \otimes \rho_{2} \otimes \cdots \otimes \rho_{N}\right)\right]=0
$$

for all infinitesimal variations

$$
\begin{aligned}
& \delta\left(\rho_{1} \otimes \rho_{2} \otimes \cdots \otimes \rho_{N}\right)=\delta \rho_{1} \otimes I_{d_{2}} \otimes \cdots \otimes I_{d_{N}}+I_{d_{1}} \otimes \delta_{\rho_{2}} \otimes I_{d_{3}} \otimes \cdots \otimes I_{d_{N}} \\
& +\cdots+I_{d_{1}} \otimes I_{d_{2}} \otimes \cdots \otimes I_{d_{N-1}} \otimes \delta \rho_{N},
\end{aligned}
$$

subjected to the constraint $\operatorname{det}\left(I_{d_{i}}+\delta \rho_{i}\right)=1$, which is equivalent to $\operatorname{Tr}\left[\delta \rho_{i}\right]=0, i=1,2, \ldots, N$, using $\operatorname{det}\left(e^{A}\right)=e^{\operatorname{Tr}[A]}$ for a given matrix $A$. Thus, $\delta \rho_{i}$ can be represented by the $S U$ generators, $\delta \rho_{i}=\sum_{k} \delta c_{k}^{i} \lambda_{k}^{i}$. It follows that $\operatorname{Tr}\left[\tilde{\rho} \lambda_{\alpha_{k}}^{\left\{\mu_{k}\right\}}\right]=0$ for any $\alpha_{k}$ and $\mu_{k}$. Hence the terms proportional to $\lambda_{\alpha_{k}}^{\left\{\mu_{k}\right\}}$ in (2.69) disappear.

Corollary 2.11. The normal form of a product state in $\mathcal{d}$ must be proportional to the identity.

Proof. Let $\rho$ be such a state. From (2.74), we get that

$$
\tilde{\rho}_{i}=\operatorname{Tr}_{1,2, \ldots, i-1, i+1, \ldots, N}[\rho]=\frac{1}{d_{i}} I_{d_{i}}
$$

Therefore for a product state $\rho$ we have

$$
\tilde{\rho}=\rho_{1} \otimes \rho_{2} \otimes \cdots \otimes \rho_{N}=\frac{1}{\prod_{i=1}^{N} d_{i}} \otimes_{i=1}^{N} I_{d_{i}} .
$$

As an example for separability of multipartite states in terms of their normal forms (2.74), we consider the PPT entangled edge state [79]

$$
\rho=\left(\begin{array}{cccccccc}
1 & 0 & 0 & 0 & 0 & 0 & 0 & 1 \\
0 & a & 0 & 0 & 0 & 0 & 0 & 0 \\
0 & 0 & b & 0 & 0 & 0 & 0 & 0 \\
0 & 0 & 0 & c & 0 & 0 & 0 & 0 \\
0 & 0 & 0 & 0 & \frac{1}{c} & 0 & 0 & 0 \\
0 & 0 & 0 & 0 & 0 & \frac{1}{b} & 0 & 0 \\
0 & 0 & 0 & 0 & 0 & 0 & \frac{1}{a} & 0 \\
1 & 0 & 0 & 0 & 0 & 0 & 0 & 1
\end{array}\right)
$$


mixed with noises

$$
\rho_{p}=p \rho+\frac{(1-p)}{8} I_{8}
$$

Select $a=2, b=3$, and $c=0.6$. Using the criterion in [97] we get that $\rho_{p}$ is entangled for $0.92744<p \leq 1$. But after transforming $\rho_{p}$ to its normal form (2.74), the criterion can detect entanglement for $0.90285<p \leq 1$.

Here we indicate that the filtering transformation does not change the PPT property. Let $\rho \in \mathscr{L}_{A} \otimes \mathscr{L}_{B}$ be PPT, that is, $\rho^{T_{A}} \geq 0$, and $\rho^{T_{B}} \geq 0$. Let $\tilde{\rho}$ be the normal form of $\rho$. From (2.61) we have

$$
\tilde{\rho}^{T_{A}}=\frac{\left(F_{A}^{*} \otimes F_{B}\right) \rho^{T_{A}}\left(F_{A}^{T} \otimes F_{B}^{\dagger}\right)}{\operatorname{Tr}\left[\left(F_{A} \otimes F_{B}\right) \rho\left(F_{A} \otimes F_{B}\right)^{\dagger}\right]} .
$$

For any vector $|\psi\rangle$, we have

$$
\left\langle\psi\left|\widetilde{\rho}^{T_{A}}\right| \psi\right\rangle=\frac{\left\langle\psi\left|\left(F_{A}^{*} \otimes F_{B}\right) \rho^{T_{A}}\left(F_{A}^{T} \otimes F_{B}^{\dagger}\right)\right| \psi\right\rangle}{\operatorname{Tr}\left[\left(F_{A} \otimes F_{B}\right) \rho\left(F_{A} \otimes F_{B}\right)^{\dagger}\right]} \equiv\left\langle\psi^{\prime}\left|\rho^{T_{A}}\right| \psi^{\prime}\right\rangle \geq 0,
$$

where $\left|\psi^{\prime}\right\rangle=\left(F_{A}^{T} \otimes F_{B}^{\dagger}\right)|\psi\rangle / \sqrt{\operatorname{Tr}\left[\left(F_{A} \otimes F_{B}\right) \rho\left(F_{A} \otimes F_{B}\right)^{\dagger}\right]} \cdot \tilde{\rho}^{T_{B}} \geq 0$ can be proved similarly. This property is also valid for multipartite case. Hence a bound entangled state will be bound entangled under filtering transformations.

\subsection{Entanglement Witness Based on Correlation Matrix Criterion}

Entanglement witness (EW) is another way to describe separability. Based on CM criterion we can further construct entanglement witness in terms of LOOs. EW [92] is an observable of the composite system such that (i) nonnegative expectation values in all separable states and (ii) at least one negative eigenvalue (can recognize at least one entangled state). Consider bipartite systems in $\mathfrak{\ell}_{A}^{M} \otimes \mathscr{\ell}_{B}^{N}$ with $M \leq N$.

Theorem 2.12. For any properly selected LOOs $G_{k}^{A}$ and $G_{k}^{B}$,

$$
W=I-\alpha \sum_{k=0}^{N^{2}-1} G_{k}^{A} \otimes G_{k}^{B}
$$

is an $E W[98]$, where $\alpha=\sqrt{M N} /(\sqrt{(M-1)(N-1)}+1)$ and

$$
G_{0}^{A}=\frac{1}{\sqrt{M}} I_{M}, \quad G_{0}^{B}=\frac{1}{\sqrt{N}} I_{N} .
$$


Proof. Let $\rho=\sum_{l, m=0}^{N^{2}-1} T_{l m} \lambda_{l}^{A} \otimes \lambda_{m}^{B}$ be a separable state, where $\lambda_{k}^{A / B}$ are normalized generators of $\operatorname{SU}(M / N)$ with $\lambda_{0}^{A}=(1 / \sqrt{M}) I_{M}, \lambda_{0}^{B}=(1 / \sqrt{N}) I_{N}$. Any other LOOs $G_{k}^{A / B}$ fulfilling (2.88) can be obtained from these $\lambda$ s through orthogonal transformations $\mathcal{O}^{A / B}, G_{k}^{A / B}=$ $\sum_{l=0}^{N^{2}-1} \mathcal{O}_{k l}^{A / B} \lambda_{l}$, where $\mathcal{O}^{A / B}=\left(\begin{array}{cc}1 & 0 \\ 0 & R^{A / B}\end{array}\right)$ and $\mathcal{R}^{A / B}$ are $\left(N^{2}-1\right) \times\left(N^{2}-1\right)$ orthogonal matrices. We have

$$
\begin{aligned}
\operatorname{Tr}[\rho W] & =1-\alpha \frac{1}{\sqrt{M N}}-\alpha \sum_{k=1}^{N^{2}-1} \sum_{l, m=1}^{N^{2}-1} R_{k l}^{A} \mathcal{R}_{k m}^{B} \operatorname{Tr}\left[\rho\left(\lambda_{l}^{A} \otimes \lambda_{m}^{B}\right)\right] \\
& =\frac{\sqrt{(M-1)(N-1)}}{\sqrt{(M-1)(N-1)}+1}-\frac{1}{\sqrt{M N}(\sqrt{(M-1)(N-1)}+1)} \sum_{k=1}^{N^{2}-1} \sum_{l, m=1}^{N^{2}-1} R_{k l}^{A} T_{l m} \mathcal{R}_{k m}^{B} \\
& \geq \frac{\sqrt{M N(M-1)(N-1)}-\|T\|_{K F}}{\sqrt{M N}(\sqrt{(M-1)(N-1)}+1)} \geq 0
\end{aligned}
$$

where we have used $\operatorname{Tr}[R T] \leq\|T\|_{K F}$ for any unitary $\mathcal{R}$ in the first inequality and the $\mathrm{CM}$ criterion in the second inequality.

Now let $\rho=(1 / M N)\left(I_{M N}+\sum_{i=1}^{M^{2}-1} s_{i} \lambda_{i}^{A} \otimes I_{N}+\sum_{j=1}^{N^{2}-1} r_{j} I_{M} \otimes \lambda_{j}^{B}+\sum_{i=1}^{M^{2}-1} \sum_{j=1}^{N^{2}-1} T_{i j} \lambda_{i}^{A} \otimes\right.$ $\left.\lambda_{j}^{B}\right)$ be a state in $\mathscr{\ell}_{A}^{M} \otimes \mathscr{\ell}_{B}^{N}$ which violates the CM criterion. Denote $\sigma_{k}(T)$ as the singular values of $T$. By singular value decomposition, one has $T=U^{\dagger} \Lambda V^{*}$, where $\Lambda$ is a diagonal matrix with $\Lambda_{k k}=\sigma_{k}(T)$. Now choose LOOs to be $G_{k}^{A}=\sum_{l} U_{k l} \lambda_{l}^{A}, G_{k}^{B}=\sum_{m} V_{k m} \lambda_{m}^{B}$ for $k=1,2, \ldots, N^{2}-1$ and $G_{0}^{A}=(1 / M) I_{M}, G_{0}^{B}=(1 / N) I_{N}$. We obtain

$$
\begin{aligned}
\operatorname{Tr}[\rho W] & =1-\alpha \frac{1}{\sqrt{M N}}-\alpha \sum_{k=1}^{N^{2}-1} \sum_{l, m=1}^{N^{2}-1} U_{k l} V_{k m} \operatorname{Tr}\left[\rho\left(\lambda_{l}^{A} \otimes \lambda_{m}^{B}\right)\right] \\
& =\frac{\sqrt{(M-1)(N-1)}}{\sqrt{(M-1)(N-1)}+1}-\frac{1}{\sqrt{M N}(\sqrt{(M-1)(N-1)}+1)} \operatorname{Tr}\left[U T V^{T}\right] \\
& =\frac{\sqrt{M N(M-1)(N-1)}-\|T\|_{K F}}{\sqrt{M N}(\sqrt{(M-1)(N-1)}+1)}<0
\end{aligned}
$$

where the $\mathrm{CM}$ criterion has been used in the last step.

As the CM criterion can be generalized to multipartite form [97], we can also define entanglement witness for multipartite system in $\mathscr{\ell}_{1}^{d_{1}} \otimes \mathscr{H}_{2}^{d_{2}} \otimes \cdots \otimes \mathscr{H}_{N}^{d_{N}}$. Set $d(M)=\max \left\{d_{\mu_{i}}, i=\right.$ $1,2, \ldots, M\}$. Choose LOOs $G_{k}^{\left\{\mu_{i}\right\}}$ for $0 \leq k \leq d(M)^{2}-1$ with $G_{0}^{\left\{\mu_{i}\right\}}=\left(1 / d_{\mu_{i}}\right) I_{d_{\mu_{i}}}$ and define

$$
W^{(M)}=I-\beta^{(M)} \sum_{k=0}^{d(M)^{2}-1} G_{k}^{\left\{\mu_{1}\right\}} \otimes G_{k}^{\left\{\mu_{2}\right\}} \otimes \cdots \otimes G_{k}^{\left\{\mu_{M}\right\}},
$$


where $\beta^{(M)}=\sqrt{\prod_{i=1}^{M} d_{\mu_{i}}} /\left(1+\sqrt{\prod_{i=1}^{M}\left(d_{\mu_{i}}-1\right)}\right), 2 \leq M \leq N$. One can prove that (2.91) is an EW candidate for multipartite states. First we assume that $\left\|\tau^{(M)}\right\|_{K F}=\left\|\tau_{\left(m_{0}\right)}\right\|_{K F^{2}}$. Note that, for any $\tau_{\left(m_{0}\right)}$, there must exist an elementary transformation $P$ such that $\sum_{k=1}^{d(M)^{2}-1} \tau_{k k \cdots k}^{\left\{\mu_{1} \mu_{2} \cdots \mu_{M}\right\}}=$ $\operatorname{Tr}\left[\tau_{\left(m_{0}\right)} P\right]$. Then for an N-partite separable state we have

$$
\begin{aligned}
\operatorname{Tr}\left[\rho W^{(M)}\right] & =1-\beta^{(M)} \frac{1}{\sqrt{\prod_{i=1}^{M} d_{\mu_{i}}}}-\beta^{(M)} \frac{1}{\prod_{i=1}^{M} d_{\mu_{i}}} \operatorname{Tr}\left[\tau_{\left(m_{0}\right)} P\right] \\
& \geq 1-\beta^{(M)} \frac{1}{\sqrt{\prod_{i=1}^{M} d_{\mu_{i}}}}-\beta^{(M)} \frac{1}{\prod_{i=1}^{M} d_{\mu_{i}}}\left\|\tau_{\left(m_{0}\right)}\right\|_{K F} \\
& \geq 1-\beta^{(M)} \frac{1}{\sqrt{\prod_{i=1}^{M} d_{\mu_{i}}}}-\beta^{(M)} \frac{1}{\prod_{i=1}^{M} d_{\mu_{i}}} \sqrt{\prod_{k=1}^{M} d_{\mu_{k}}\left(d_{\mu_{k}}-1\right)} \\
& =0
\end{aligned}
$$

for any $2 \leq M \leq N$, where we have taken into account that $P$ is orthogonal and $\operatorname{Tr}[M U] \leq\|M\|_{K F}$ for any unitary $U$ at the first inequality. The second inequality is due to the generalized CM criterion.

By choosing proper LOOs, it is also easy to show that $W^{(M)}$ has negative eigenvalues. For example, for three-qubit case, taking the normalized Pauli matrices as LOOs, one finds a negative eigenvalue of $W^{(M)},(1-\sqrt{3}) / 2$.

\section{Concurrence and Tangle}

In this section, we focus on two important measures: concurrence and tangle (see $[99,100])$. An elegant formula for concurrence of two-qubit states is derived analytically by Wootters $[53,101]$. This quantity has recently been shown to play an essential role in describing quantum phase transition in various interacting quantum many-body systems $[102,103]$ and may affect macroscopic properties of solids significantly [104, 105]. Furthermore, concurrence also provides an estimation [106, 107] for the entanglement of formation (EOF) [76], which quantifies the required minimally physical resources to prepare a quantum state.

Let $\mathscr{L}_{A}$ (resp., $\mathscr{d}_{B}$ ) be an $M$-(resp., $N$-) dimensional complex vector space with $|i\rangle, i=$ $1, \ldots, M$ (resp., $|j\rangle, j=1, \ldots, N)$, as an orthonormal basis. A general pure state on $\mathscr{d}_{A} \otimes \mathscr{L}_{B}$ is of the form

$$
|\Psi\rangle=\sum_{i=1}^{M} \sum_{j=1}^{N} a_{i j}|i\rangle \otimes|j\rangle,
$$

where $a_{i j} \in \mathbb{C}$ satisfy the normalization $\sum_{i=1}^{M} \sum_{j=1}^{N} a_{i j} a_{i j}^{*}=1$.

The concurrence of (3.1) is defined by [27, 108, 109]

$$
C(|\psi\rangle)=\sqrt{2\left(1-\operatorname{Tr}\left[\rho_{A}^{2}\right]\right)}
$$


where $\rho_{A}=\operatorname{Tr}_{B}[|\psi\rangle\langle\psi|]$. The definition is extended to general mixed states $\rho=\sum_{i} p_{i}\left|\psi_{i}\right\rangle\left\langle\psi_{i}\right|$ by the convex roof

$$
C(\rho)=\min _{\left.\left\{p_{i}, \psi_{i}\right\rangle\right\}} \sum_{i} p_{i} C\left(\left|\psi_{i}\right\rangle\right)
$$

For two-qubits systems, the concurrence of $|\Psi\rangle$ is given by

$$
C(|\Psi\rangle)=|\langle\Psi \mid \tilde{\Psi}\rangle|=2\left|a_{11} a_{22}-a_{12} a_{21}\right|
$$

where $|\tilde{\Psi}\rangle=\sigma_{y} \otimes \sigma_{y}\left|\Psi^{*}\right\rangle,\left|\Psi^{*}\right\rangle$ is the complex conjugate of $|\Psi\rangle$, and $\sigma_{y}$ is the Pauli matrix, $\sigma_{y}=\left(\begin{array}{cc}0 & -i \\ i & 0\end{array}\right)$.

For a mixed two-qubit quantum state $\rho$, the entanglement of formation $E(\rho)$ has a simple relation with the concurrence $[53,101]$

$$
E(\rho)=h\left(\frac{1+\sqrt{1-C(\rho)^{2}}}{2}\right)
$$

where $h(x)=-x \log _{2} x-(1-x) \log _{2}(1-x)$,

$$
C(\rho)=\max \left\{\lambda_{1}-\lambda_{2}-\lambda_{3}-\lambda_{4}, 0\right\},
$$

where the $\lambda_{i} \mathrm{~s}$ are the eigenvalues, in decreasing order, of the Hermitian matrix $\sqrt{\sqrt{\rho} \tilde{\rho} \sqrt{\rho}}$ and $\tilde{\rho}=\left(\sigma_{y} \otimes \sigma_{y}\right) \rho^{*}\left(\sigma_{y} \otimes \sigma_{y}\right)$.

Another entanglement measure called tangle is defined by

$$
\tau(|\psi\rangle)=C^{2}(|\psi\rangle)=2\left(1-\operatorname{Tr}\left[\rho_{A}^{2}\right]\right)
$$

for a pure state $|\psi\rangle$. For mixed state $\rho=\sum_{i} p_{i}\left|\psi_{i}\right\rangle\left\langle\psi_{i}\right|$, the definition is given by

$$
\tau(\rho)=\min _{\left\{p_{i},\left|\psi_{i}\right\rangle\right\}} \sum_{i} p_{i} \tau\left(\left|\psi_{i}\right\rangle\right)
$$

For multipartite state $|\psi\rangle \in \mathscr{H}_{1} \otimes \mathscr{H}_{2} \otimes \cdots \otimes \mathscr{H}_{N}, \operatorname{dim} \mathscr{H}_{i}=d_{i}, i=1, \ldots, N$, the concurrence of $|\psi\rangle$ is defined by $[110,111]$

$$
C_{N}(|\psi\rangle\langle\psi|)=2^{1-N / 2} \sqrt{\left(2^{N}-2\right)-\sum_{\alpha} \operatorname{Tr}\left[\rho_{\alpha}^{2}\right]}
$$

where $\alpha$ labels all different reduced density matrices. 
Up to constant factor (3.9) can be also expressed in another way. Let $H$ denote a $d$ dimensional vector space with basis $|i\rangle, i=1,2, \ldots, d$. An $N$-partite pure state in $H \otimes \cdots \otimes H$ is generally of the form

$$
|\Psi\rangle=\sum_{i_{1}, i_{2}, \ldots i_{N}=1}^{d} a_{i_{1}, i_{2}, \ldots i_{N}}\left|i_{1}, i_{2}, \ldots i_{N}\right\rangle, \quad a_{i_{1}, i_{2}, \ldots i_{N}} \in \mathbb{C}
$$

Let $\alpha$ and $\alpha^{\prime}$ (resp., $\beta$ and $\beta^{\prime}$ ) be subsets of the subindices of $a$, associated to the same sub Hilbert spaces but with different summing indices. $\alpha$ (or $\alpha^{\prime}$ ) and $\beta$ (or $\beta^{\prime}$ ) span the whole space of the given subindix of $a$. The generalized concurrence of $|\Psi\rangle$ is then given by [27]

$$
C_{d}^{N}(|\Psi\rangle)=\sqrt{\frac{d}{2 m(d-1)} \sum_{p} \sum_{\left\{\alpha, \alpha^{\prime}, \beta, \beta^{\prime}\right\}}^{d}\left|a_{\alpha \beta} a_{\alpha^{\prime} \beta^{\prime}}-a_{\alpha \beta^{\prime}} a_{\alpha^{\prime} \beta}\right|^{2}},
$$

where $m=2^{N-1}-1$ and $\sum_{p}$ stands for the summation over all possible combinations of the indices of $\alpha$ and $\beta$.

For a mixed multipartite quantum state, $\rho=\sum_{i} p_{i}\left|\psi_{i}\right\rangle\left\langle\psi_{i}\right|$ in $\mathscr{\ell}_{1} \otimes \mathscr{l}_{2} \otimes \cdots \otimes \mathscr{\ell}_{N}$, the corresponding concurrence is given by the convex roof:

$$
C_{N}(\rho)=\min _{\left.\left\{p_{i}, \psi_{i}\right\}\right\rangle} \sum_{i} p_{i} C_{N}\left(\left|\psi_{i}\right\rangle\right)
$$

\subsection{Lower and Upper Bounds of Concurrence}

Calculations of the concurrence for general mixed states are extremely difficult. However, one can try to find the lower and the upper bounds to estimate the exact values of the concurrence $[46,61,64,65]$.

\subsubsection{Lower Bound of Concurrence from Covariance Matrix Criterion}

In [61] a lower bound of $C(\rho)$ has been obtained as

$$
C(\rho) \geq \sqrt{\frac{2}{M(M-1)}}\left[\operatorname{Max}\left(\left\|T_{A}(\rho)\right\|,\|R(\rho)\|\right)-1\right]
$$

where $T_{A}$ and $R$ stand for partial transpose with respect to subsystem $A$ and the realignment, respectively. This bound is further improved based on local uncertainty relations [64]

$$
C(\rho) \geq \frac{M+N-2-\sum_{i} \Delta_{\rho}^{2}\left(G_{i}^{A} \otimes I+I \otimes G_{i}^{B}\right)}{\sqrt{2 M(M-1)}},
$$

where $G_{i}^{A}$ and $G_{i}^{B}$ are any set of local orthonormal observables, $\Delta_{\rho}^{2}(X)=\operatorname{Tr}\left[X^{2} \rho\right]-(\operatorname{Tr}[X \rho])^{2}$. 
Bound (3.14) again depends on the choice of the local orthonormal observables. This bound can be optimized, in the sense that a local orthonormal observable-independent up bound of the right-hand side of (3.14) can be obtained.

Theorem 3.1. Let $\rho$ be a bipartite state in $\mathfrak{l}_{M}^{A} \otimes \mathfrak{l}_{N}^{B}$. Then $C(\rho)$ satisfies [90]

$$
C(\rho) \geq \frac{2\|C\|_{K F}-\left(1-\operatorname{Tr}\left[\rho_{A}^{2}\right]\right)-\left(1-\operatorname{Tr}\left[\rho_{B}^{2}\right]\right)}{\sqrt{2 M(M-1)}} .
$$

Proof. The other orthonormal normalized basis of the local orthonormal observable space can be obtained from $A_{i}$ and $B_{i}$ by unitary transformations $U$ and $V: \widetilde{A}_{i}=\sum_{l} U_{i l} A_{l}$ and $\widetilde{B}_{j}=\sum_{m} V_{j m}^{*} B_{m}$. Select $U$ and $V$ so that $C=U^{\dagger} \Lambda V$ is the singular value decomposition of $C$. Then the new observables can be written as $\widetilde{A}_{i}=\sum_{l} U_{i l} A_{l}, \widetilde{B}_{j}=-\sum_{m} V_{j m}^{*} B_{m}$. We have

$$
\begin{aligned}
\sum_{i} \Delta_{\rho}^{2}\left(\tilde{A}_{i} \otimes I+I \otimes \widetilde{B}_{i}\right) & =\sum_{i}\left[\Delta_{\rho_{A}}^{2}\left(\tilde{A}_{i}\right)+\Delta_{\rho_{A}}^{2}\left(\tilde{B}_{i}\right)+2\left(\left\langle\tilde{A}_{i} \otimes \tilde{B}_{i}\right\rangle-\left\langle\tilde{A}_{i}\right\rangle\left\langle\widetilde{B}_{i}\right\rangle\right)\right] \\
& =M-\operatorname{Tr}\left[\rho_{A}^{2}\right]+N-\operatorname{Tr}\left[\rho_{B}^{2}\right]-2 \sum_{i}\left(U C V^{\dagger}\right)_{i i} \\
& =M-\operatorname{Tr}\left[\rho_{A}^{2}\right]+N-\operatorname{Tr}\left[\rho_{B}^{2}\right]-2\|C\|_{K F} .
\end{aligned}
$$

Substituting above relation to (3.14), one gets (3.15).

Bound (3.15) does not depend on the choice of local orthonormal observables. It can be easily applied and realized by direct measurements in experiments. It is in accord with the result in [46] where optimization of entanglement witness based on local uncertainty relation has been taken into account. As an example, let us consider the $3 \times 3$ bound entangled state [76]

$$
\rho=\frac{1}{4}\left(I_{9}-\sum_{i=0}^{4}\left|\xi_{i}\right\rangle\left\langle\xi_{i}\right|\right)
$$

where $I_{9}$ is the $9 \times 9$ identity matrix, $\left|\xi_{0}\right\rangle=(1 / \sqrt{2})|0\rangle(|0\rangle-|1\rangle),\left|\xi_{1}\right\rangle=(1 / \sqrt{2})(|0\rangle-|1\rangle)|2\rangle,\left|\xi_{2}\right\rangle=$ $(1 / \sqrt{2})|2\rangle(|1\rangle-|2\rangle),\left|\xi_{3}\right\rangle=(1 / \sqrt{2})(|1\rangle-|2\rangle)|0\rangle$, and $\left|\xi_{4}\right\rangle=(1 / 3)(|0\rangle+|1\rangle+|2\rangle)(|0\rangle+|1\rangle+|2\rangle)$. We simply choose the local orthonormal observables to be the normalized generators of SU(3). Formula (3.13) gives $C(\rho) \geq 0.050$. Formula (3.14) gives $C(\rho) \geq 0.052$ [64], while formula (3.15) yields a better lower bound $C(\rho) \geq 0.0555$.

If we mix the bound entangled state (3.17) with $|\psi\rangle=(1 / \sqrt{3}) \sum_{i=0}^{2}|i i\rangle$, that is,

$$
\rho^{\prime}=(1-x) \rho+x|\psi\rangle\langle\psi|
$$

then it is easily seen that (3.15) gives a better lower bound of concurrence than formula (3.13) (Figure 1). 


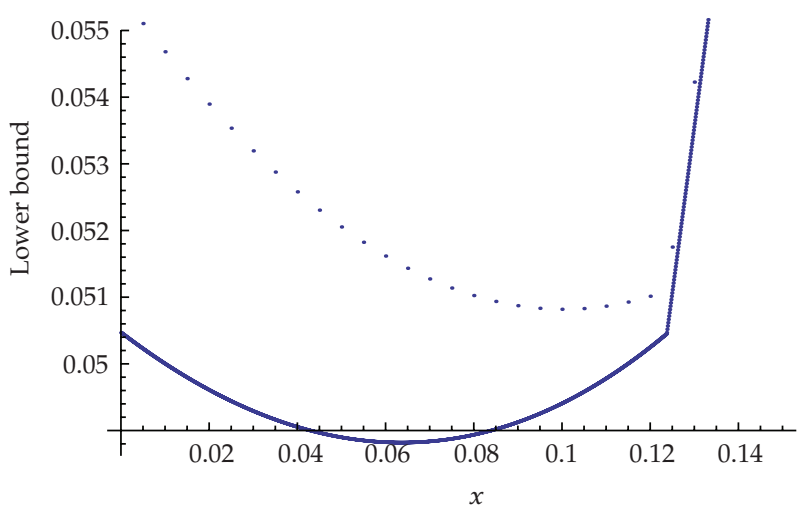

Figure 1: Lower bounds from (3.15) (dashed line) and (3.13) (solid line) for mixed state (3.18).

\subsubsection{Lower Bound of Concurrence from "Two-Qubit" Decomposition}

In [67] the authors derived an analytical lower bound of concurrence for arbitrary bipartite quantum states by decomposing the joint Hilbert space into many $2 \otimes 2$ dimensional subspaces, which does not involve any optimization procedure and gives an effective evaluation of entanglement together with an operational sufficient condition for the distill ability of any bipartite quantum states.

\section{(1) Lower Bound of Concurrence for Bipartite States}

The lower bound $\tau_{2}$ of concurrence for bipartite states has been obtained in [67]. For a bipartite quantum state $\rho$ in $H \otimes H$, the concurrence $C(\rho)$ satisfies

$$
\tau_{2}(\rho) \equiv \frac{d}{2(d-1)} \sum_{m, n=1}^{d(d-1) / 2} C_{m n}^{2}(\rho) \leq C^{2}(\rho)
$$

where $C_{m n}(\rho)=\max \left\{0, \lambda_{m n}^{(1)}-\lambda_{m n}^{(2)}-\lambda_{m n}^{(3)}-\lambda_{m n}^{(4)}\right\}$ with $\lambda_{m n}^{(1)}, \ldots, \lambda_{m n}^{(4)}$ being the square roots of the four nonzero eigenvalues, in decreasing order, of the non-Hermitian matrix $\rho \tilde{\rho}_{m n}$ with $\tilde{\rho}_{m n}=\left(L_{m} \otimes L_{n}\right) \rho^{*}\left(L_{m} \otimes L_{n}\right)$, while $L_{m}$ and $L_{n}$ are the generators of $S O(d)$.

The lower bound $\tau_{2}$ in (3.19) in fact characterizes all two qubits' entanglement in a high dimensional bipartite state. One can directly verify that there are at most $4 \times 4=16$ nonzero elements in each matrix $\rho \tilde{\rho}_{m n}$. These elements constitute a $4 \times 4$ matrix $\rho\left(\sigma_{y} \otimes \sigma_{y}\right) \rho^{*}\left(\sigma_{y} \otimes \sigma_{y}\right)$, where $\sigma_{y}$ is the Pauli matrix, the matrix $\rho$ is a submatrix of the original $\rho$ :

$$
\rho=\left(\begin{array}{cccc}
\rho_{i k, i k} & \rho_{i k, i l} & \rho_{i k, j k} & \rho_{i k, j l} \\
\rho_{i l, i k} & \rho_{i l, i l} & \rho_{i l, j k} & \rho_{i l, j l} \\
\rho_{j k, i k} & \rho_{j k, i l} & \rho_{j k, j k} & \rho_{j k, j l} \\
\rho_{j l, i k} & \rho_{j l, i l} & \rho_{j l, j k} & \rho_{j l, j l}
\end{array}\right),
$$


$i \neq j$, and $k \neq l$, with subindices $i$ and $j$ associated with the first space, $k$ and $l$ with the second space. The two-qubit submatrix $\rho$ is not normalized but positive semidefinite. $\mathcal{C}_{m n}$ are just the concurrences of these states (3.20).

The bound $\tau_{2}$ provides a much clearer structure of entanglement, which not only yields an effective separability criterion and an easy evaluation of entanglement, but also helps one to classify mixed-state entanglement.

\section{(2) Lower Bound of Concurrence for Multipartite States}

We first consider tripartite case. A general pure state on $H \otimes H \otimes H$ is of the form

$$
|\Psi\rangle=\sum_{i, j, k=1}^{d} a_{i j k}|i j k\rangle, \quad a_{i j k} \in \mathbb{C}, \quad \sum_{i, j, k=1}^{d} a_{i j k} a_{i j k}^{*}=1
$$

with

$$
\begin{aligned}
& C_{d}^{3}(|\Psi\rangle) \\
& =\sqrt{\frac{d}{6(d-1)}} \sqrt{\sum\left(\left|a_{i j k} a_{p q m}-a_{i j m} a_{p q k}\right|^{2}+\left|a_{i j k} a_{p q m}-a_{i q k} a_{p j m}\right|^{2}+\left|a_{i j k} a_{p q m}-a_{p j k} a_{i q m}\right|^{2}\right)},
\end{aligned}
$$

or equivalently

$$
C_{d}^{3}(|\Psi\rangle)=\sqrt{\frac{d}{6(d-1)}\left(3-\left(\operatorname{Tr}\left[\rho_{1}^{2}\right]+\operatorname{Tr}\left[\rho_{2}^{2}\right]+\operatorname{Tr}\left[\rho_{3}^{2}\right]\right)\right)},
$$

where $\rho_{1}=\operatorname{Tr}_{23}[\rho], \rho_{2}=\operatorname{Tr}_{13}[\rho]$, and $\rho_{3}=\operatorname{Tr}_{12}[\rho]$ are the reduced density matrices of $\rho=$ $|\Psi\rangle\langle\Psi|$.

Define $C_{\alpha \beta}^{12 \mid 3}(|\Psi\rangle)=\left|a_{i j k} a_{p q m}-a_{i j m} a_{p q k}\right|, C_{\alpha \beta}^{13 \mid 2}(|\Psi\rangle)=\left|a_{i j k} a_{p q m}-a_{i q k} a_{p j m}\right|$, and $C_{\alpha \beta}^{23 \mid 1}(|\Psi\rangle)=\left|a_{i j k} a_{p q m}-a_{p j k} a_{i q m}\right|$, where $\alpha$ and $\beta$ of $C_{\alpha \beta}^{12 \mid 3}$ (resp., $C_{\alpha \beta}^{13 \mid 2}$, resp., $C_{\alpha \beta}^{23 \mid 1}$ ) stand for the subindices of $a$ associated with the subspaces 1, 2, and 3 (resp., 1, 3, and 2; resp., 2, 3, and $1)$. Let $L^{i_{1} i_{2} \cdots i_{N}}$ denote the generators of group $S O\left(d_{i_{1}} d_{i_{2}} \cdots d_{i_{N}}\right)$ associated to the subsystems $i_{1}, i_{2}, \ldots, i_{N}$. Then for a tripartite pure state (3.21), one has

$$
\begin{aligned}
C_{d}^{3}(|\Psi\rangle) & =\sqrt{\frac{d}{6(d-1)} \sum_{\alpha}^{d^{2}\left(d^{2}-1\right) / 2} \sum_{\beta}^{d(d-1) / 2}\left[\left(C_{\alpha \beta}^{12 \mid 3}(|\Psi\rangle)\right)^{2}+\left(C_{\alpha \beta}^{13 \mid 2}(|\Psi\rangle)\right)^{2}+\left(C_{\alpha \beta}^{23 \mid 1}(|\Psi\rangle)\right)^{2}\right]} \\
& =\sqrt{\frac{d}{6(d-1)} \sum_{\alpha \beta}\left[\left(\left|\left\langle\Psi\left|S_{\alpha \beta}^{12 \mid 3}\right| \Psi^{*}\right\rangle\right|\right)^{2}+\left(\left|\left\langle\Psi\left|S_{\alpha \beta}^{13 \mid 2}\right| \Psi^{*}\right\rangle\right|\right)^{2}+\left(\left|\left\langle\Psi\left|S_{\alpha \beta}^{23 \mid 2}\right| \Psi^{*}\right\rangle\right|\right)^{2}\right]},
\end{aligned}
$$

where $S_{\alpha \beta}^{12 \mid 3}=\left(L_{\alpha}^{12} \otimes L_{\beta}^{3}\right), S_{\alpha \beta}^{13 \mid 2}=\left(L_{\alpha}^{13} \otimes L_{\beta}^{2}\right)$, and $S_{\alpha \beta}^{23 \mid 1}=\left(L_{\beta}^{1} \otimes L_{\alpha}^{23}\right)$. 
Theorem 3.2. For an arbitrary mixed state $\rho$ in $H \otimes H \otimes H$, the concurrence $C(\rho)$ satisfies [112]

$$
\tau_{3}(\rho) \equiv \frac{d}{6(d-1)} \sum_{\alpha}^{d^{2}\left(d^{2}-1\right) / 2} \sum_{\beta}^{d(d-1) / 2}\left[\left(C_{\alpha \beta}^{12 \mid 3}(\rho)\right)^{2}+\left(C_{\alpha \beta}^{13 \mid 2}(\rho)\right)^{2}+\left(C_{\alpha \beta}^{23 \mid 1}(\rho)\right)^{2}\right] \leq C^{2}(\rho),
$$

where $\tau_{3}(\rho)$ is a lower bound of $C(\rho)$

$$
C_{\alpha \beta}^{12 \mid 3}(\rho)=\max \left\{0, \lambda(1)_{\alpha \beta}^{12 \mid 3}-\lambda(2)_{\alpha \beta}^{12 \mid 3}-\lambda(3)_{\alpha \beta}^{12 \mid 3}-\lambda(4)_{\alpha \beta}^{12 \mid 3}\right\}
$$

where $\lambda(1)_{\alpha \beta}^{12 \mid 3}, \lambda(2)_{\alpha \beta}^{12 \mid 3}, \lambda(3)_{\alpha \beta}^{12 \mid 3}$, and $\lambda(4)_{\alpha \beta}^{12 \mid 3}$ are the square roots of the four nonzero eigenvalues, in decreasing order, of the non-Hermitian matrix $\rho \tilde{\rho}_{\alpha \beta}^{12 \mid 3}$ with $\tilde{\rho}_{\alpha \beta}^{12 \mid 3}=S_{\alpha \beta}^{12 \mid 3} \rho^{*} S_{\alpha \beta}^{12 \mid 3} \cdot C_{\alpha \beta}^{13 \mid 2}(\rho)$ and $C_{\alpha \beta}^{23 \mid 1}(\rho)$ are defined in a similar way to $C_{\alpha \beta}^{12 \mid 3}(\rho)$.

Proof. Set $\left|\xi_{i}\right\rangle=\sqrt{p_{i}}\left|\psi_{i}\right\rangle, x_{\alpha \beta}^{i}=\left|\left\langle\xi_{i}\left|S_{\alpha \beta}^{12 \mid 3}\right| \xi_{i}^{*}\right\rangle\right|, y_{\alpha \beta}^{i}=\left|\left\langle\xi_{i}\left|S_{\alpha \beta}^{13 \mid 2}\right| \xi_{i}^{*}\right\rangle\right|$, and $z_{\alpha \beta}^{i}=\left|\left\langle\xi_{i}\left|S_{\alpha \beta}^{1 \mid 23}\right| \xi_{i}^{*}\right\rangle\right|$. We have, from Minkowski inequality,

$$
\begin{aligned}
C(\rho) & =\min \sum_{i} \sqrt{\frac{d}{6(d-1)} \sum_{\alpha \beta}\left[\left(x_{\alpha \beta}^{i}\right)^{2}+\left(y_{\alpha \beta}^{i}\right)^{2}+\left(z_{\alpha \beta}^{i}\right)^{2}\right]} \\
& \geq \min \sqrt{\frac{d}{6(d-1)} \sum_{\alpha \beta}\left(\sum_{i}\left[\left(x_{\alpha \beta}^{i}\right)^{2}+\left(y_{\alpha \beta}^{i}\right)^{2}+\left(z_{\alpha \beta}^{i}\right)^{2}\right]^{1 / 2}\right)^{2}} .
\end{aligned}
$$

Noting that for nonnegative real variables $x_{\alpha}, y_{\alpha}, z_{\alpha}$ and given that $X=\sum_{\alpha=1}^{N} x_{\alpha}, Y=$ $\sum_{\alpha=1}^{N} Y_{\alpha}$, and $Z=\sum_{\alpha=1}^{N} z_{\alpha}$, by using Lagrange multipliers, one obtains that the following inequality holds:

$$
\sum_{\alpha=1}^{N}\left(x_{\alpha}^{2}+y_{\alpha}^{2}+z_{\alpha}^{2}\right)^{1 / 2} \geq\left(X^{2}+Y^{2}+Z^{2}\right)^{1 / 2}
$$

Therefore we have

$$
\begin{aligned}
C(\rho) & \geq \min \sqrt{\frac{d}{6(d-1)} \sum_{\alpha \beta}\left[\left(\sum_{i} x_{\alpha \beta}^{i}\right)^{2}+\left(\sum_{i} y_{\alpha \beta}^{i}\right)^{2}+\left(\sum_{i} z_{\alpha \beta}^{i}\right)^{2}\right]} \\
& \geq \sqrt{\frac{d}{6(d-1)} \sum_{\alpha \beta}\left[\left(\min \sum_{i} x_{\alpha \beta}^{i}\right)^{2}+\left(\min \sum_{i} y_{\alpha \beta}^{i}\right)^{2}+\left(\min \sum_{i} z_{\alpha \beta}^{i}\right)^{2}\right]} .
\end{aligned}
$$


The values of $C_{\alpha \beta}^{12 \mid 3}(\rho) \equiv \min \sum_{i} x_{\alpha \beta}^{i}, C_{\alpha \beta}^{13 \mid 2}(\rho) \equiv \min \sum_{i} y_{\alpha \beta}^{i}$, and $C_{\alpha \beta}^{23 \mid 1}(\rho) \equiv \min \sum_{i} z_{\alpha \beta}^{i}$ can be calculated by using the similar procedure in [53]. Here we compute the value of $C_{\alpha \beta}^{12 \mid 3}(\rho)$ in detail. The values of $C_{\alpha \beta}^{13 \mid 2}(\rho)$ and $C_{\alpha \beta}^{23 \mid 1}(\rho)$ can be obtained analogously.

Let $\lambda_{i}$ and $\left|\chi_{i}\right\rangle$ be eigenvalues and eigenvectors of $\rho$, respectively. Any decomposition of $\rho$ can be obtained from a unitary $d^{3} \times d^{3}$ matrix $V_{i j},\left|\xi_{j}\right\rangle=\sum_{i=1}^{d^{3}} V_{i j}^{*}\left(\sqrt{\lambda_{i}}\left|X_{i}\right\rangle\right)$. Therefore one has $\left\langle\xi_{i}\left|S_{\alpha \beta}^{12 \mid 3}\right| \xi_{j}^{*}\right\rangle=\left(V Y_{\alpha \beta} V^{T}\right)_{i j}$, where the matrix $Y_{\alpha \beta}$ is defined by $\left(Y_{\alpha \beta}\right)_{i j}=\left\langle x_{i}\left|S_{\alpha \beta}^{12 \mid 3}\right| x_{j}^{*}\right\rangle$. Namely, $C_{\alpha \beta}^{12 \mid 3}(\rho)=\min \sum_{i}\left|\left[V Y_{\alpha \beta} V^{T}\right]_{i i}\right|$, which has an analytical expression [53], that $C_{\alpha \beta}^{12 \mid 3}(\rho)=\max \left\{0, \lambda(1)_{\alpha \beta}^{12 \mid 3}-\sum_{j>1} \lambda(j)_{\alpha \beta}^{12 \mid 3}\right\}$, where $\lambda_{\alpha \beta}^{12 \mid 3}(k)$ are the square roots of the eigenvalues of the positive Hermitian matrix $Y_{\alpha \beta} Y_{\alpha \beta}^{\dagger}$, or equivalently the non-Hermitian matrix $\rho \tilde{\rho}_{\alpha \beta}$, in decreasing order. Here as the matrix $S_{\alpha \beta}^{12 \mid 3}$ has $d^{2}-4$ rows and $d^{2}-4$ columns that are identically zero, the matrix $\rho \tilde{\rho}_{\alpha \beta}$ has a rank not greater than 4 , that is, $\lambda_{\alpha \beta}^{12 \mid 3}(j)=0$ for $j \geq 5$. From (3.29) we have (3.25).

Theorem 3.2 can be directly generalized to arbitrary multipartite case.

Theorem 3.3. For an arbitrary $N$-partite state $\rho \in H \otimes H \otimes \cdots \otimes H$, the concurrence defined in (4.1) satisfies [112],

$$
\tau_{N}(\rho) \equiv \frac{d}{2 m(d-1)} \sum_{p} \sum_{\alpha \beta}\left(C_{\alpha \beta}^{p}(\rho)\right)^{2} \leq C^{2}(\rho)
$$

where $\tau_{N}(\rho)$ is the lower bound of $C(\rho), \sum_{p}$ stands for the summation over all possible combinations of the indices of $\alpha, \beta, C_{\alpha \beta}^{p}(\rho)=\max \left\{0, \lambda(1)_{\alpha \beta}^{p}-\lambda(2)_{\alpha \beta}^{p}-\lambda(3)_{\alpha \beta}^{p}-\lambda(4)_{\alpha \beta}^{p}\right\}$, and $\lambda(i)_{\alpha \beta}^{p}, i=1,2,3,4$, are the square roots of the four nonzero eigenvalues, in decreasing order, of the non-Hermitian matrix $\rho \tilde{\rho}_{\alpha \beta}^{p}$ where $\tilde{\rho}_{\alpha \beta}^{p}=S_{\alpha \beta}^{p} \rho^{*} S_{\alpha \beta}^{p}$.

\section{Lower Bound and Separability}

An N-partite quantum state $\rho$ is fully separable if and only if there exist $p_{i}$ with $p_{i} \geq 0, \sum_{i} p_{i}=$ 1 , and pure states $\rho_{i}^{j}=\left|\psi_{i}^{j}\right\rangle\left\langle\psi_{i}^{j}\right|$ such that

$$
\rho=\sum_{i} p_{i} \rho_{i}^{1} \otimes \rho_{i}^{2} \otimes \cdots \otimes \rho_{i}^{N}
$$

It is easily verified that, for a fully separable multipartite state $\rho, \tau_{N}(\rho)=0$. Thus $\tau_{N}(\rho)>0$ indicates that there must be some kinds of entanglement inside the quantum state, which shows that the lower bound $\tau_{N}(\rho)$ can be used to recognize entanglement.

As an example, we consider a tripartite quantum state [79] $\rho=((1-p) / 8) I_{8}+p|W\rangle\langle W|$, where $I_{8}$ is the $8 \times 8$ identity matrix, and $|W\rangle=(1 / \sqrt{3})(|100\rangle+|010\rangle+|001\rangle)$ is the tripartite $\mathrm{W}$ state. Select an entanglement witness operator to be $\mathcal{W}=(1 / 2) I_{8}-|\mathrm{GHZ}\rangle\langle\mathrm{GHZ}|$, where $|G H Z\rangle=(1 / \sqrt{2})(|000\rangle+|111\rangle)$ is to be the tripartite GHZ-state. From the condition $\operatorname{Tr}[2 \rho \rho]<$ 0 , the entanglement of $\rho$ is detected for $(3 / 5)<p \leq 1$ in [79]. In [97] the authors have obtained the generalized correlation matrix criterion which says that if an $\mathrm{N}$-qubit quantum state is fully separable, then the inequality $\left\|\tau^{N}\right\|_{K F} \leq 1$ must hold, where $\left\|\tau^{N}\right\|_{K F}=\max \left\{\left\|\tau_{n}^{N}\right\|_{K F}\right\}$, 
$\tau_{n}^{N}$ is a kind of matrix unfold of $t_{\alpha_{1} \alpha_{2} \cdots \alpha_{N}}$ defined by $t_{\alpha_{1} \alpha_{2} \cdots \alpha_{N}}=\operatorname{Tr}\left[\rho \sigma_{\alpha_{1}}^{(1)} \sigma_{\alpha_{2}}^{(2)} \cdots \sigma_{\alpha_{N}}^{(N)}\right]$, and $\sigma_{\alpha_{i}}^{(i)}$ stands for the Pauli matrix. Now using the generalized correlation, matrix criterion the entanglement of $\rho$ is detected for $0.3068<p \leq 1$. From Theorem 3.2, we have the lower bound $\tau_{3}(\rho)>0$ for $0.2727<p \leq 1$. Therefore the bound (3.71) detects entanglement better than these two criteria in this case. If we replace $W$ with GHZ state in $\rho$, then the criterion in [97] detects the entanglement of $\rho$ for $0.35355<p \leq 1$, while $\tau_{3}(\rho)$ detects, again better, the entanglement for $0.2<p \leq 1$.

Nevertheless for PPT states $\rho$, we have $\tau_{3}(\rho)=0$, which can be seen in the following way. A density matrix $\rho$ is called PPT if the partial transposition of $\rho$ over any subsystem(s) is still positive. Let $\rho^{T_{i}}$ denote the partial transposition with respect to the $i$ th subsystem. Assume that there is a PPT state $\rho$ with $\tau(\rho)>0$. Then at least one term in (3.25), say $C_{\alpha_{0} \beta_{0}}^{12 \mid 3}(\rho)$, is not zero. Define $\rho_{\alpha_{0} \beta_{0}}=L_{\alpha_{0}}^{12} \otimes L_{\beta_{0}}^{3} \rho\left(L_{\alpha_{0}}^{12} \otimes L_{\beta_{0}}^{3}\right)^{\dagger}$. By using the PPT property of $\rho$, we have

$$
\rho_{\alpha_{0} \beta_{0}}^{T_{3}}=L_{\alpha_{0}}^{12} \otimes\left(L_{\beta_{0}}^{3}\right)^{*} \rho^{T_{3}}\left(L_{\alpha_{0}}^{12}\right)^{\dagger} \otimes\left(L_{\beta_{0}}^{3}\right)^{T} \geq 0
$$

Noting that both $L_{\alpha_{0}}^{12}$ and $L_{\beta_{0}}^{3}$ are projectors to two-dimensional subsystems, $\rho_{\alpha_{0} \beta_{0}}$ can be considered as a $4 \times 4$ density matrix, while a PPT $4 \times 4$ density matrix $\rho_{\alpha_{0} \beta_{0}}$ must be a separable state, which contradicts with $C_{\alpha_{0} \beta_{0}}^{12 \mid 3}(\rho) \neq 0$.

\section{Relation between Lower Bounds of Bi- and Tripartite Concurrence}

$\tau_{3}$ is basically different from $\tau_{2}$ as $\tau_{3}$ characterizes also genuine tripartite entanglement that can not be described by bipartite decompositions. Nevertheless, there are interesting relations between them.

Theorem 3.4. For any pure tripartite state (3.21), the following inequality holds [112]:

$$
\tau_{2}\left(\rho_{12}\right)+\tau_{2}\left(\rho_{13}\right)+\tau_{2}\left(\rho_{23}\right) \leq 3 \tau_{3}(\rho)
$$

where $\tau_{2}$ is the lower bound of bipartite concurrence (3.19), $\tau_{3}$ is the lower bound of tripartite concurrence (3.25), and $\rho_{12}=\operatorname{Tr}_{3}[\rho], \rho_{13}=\operatorname{Tr}_{2}[\rho], \rho_{23}=\operatorname{Tr}_{1}[\rho]$, and $\rho=|\Psi\rangle_{123}\langle\Psi|$.

Proof. Since $C_{\alpha \beta}^{2} \leq\left(\lambda_{\alpha \beta}(1)\right)^{2} \leq \sum_{i=1}^{4}\left(\lambda_{\alpha \beta}(i)\right)^{2}=\operatorname{Tr}\left[\rho \tilde{\rho}_{\alpha \beta}\right]$ for $\rho=\rho_{12}, \rho=\rho_{13}$, and $\rho=\rho_{23}$, we have

$$
\begin{aligned}
& \tau_{2}\left(\rho_{12}\right)+\tau_{2}\left(\rho_{13}\right)+\tau_{2}\left(\rho_{23}\right) \\
& \leq \frac{d}{2(d-1)}\left(\sum_{\alpha, \beta=1}^{d(d-1) / 2} \operatorname{Tr}\left[\rho_{12}\left(\tilde{\rho}_{12}\right)_{\alpha \beta}\right]+\sum_{\alpha, \beta=1}^{d(d-1) / 2} \operatorname{Tr}\left[\rho_{13}\left(\tilde{\rho}_{13}\right)_{\alpha \beta}\right]+\sum_{\alpha, \beta=1}^{d(d-1) / 2} \operatorname{Tr}\left[\rho_{23}\left(\tilde{\rho}_{23}\right)_{\alpha \beta}\right]\right) \\
& \quad=\frac{d}{2(d-1)}\left(3-\operatorname{Tr}\left[\rho_{1}^{2}\right]-\operatorname{Tr}\left[\rho_{2}^{2}\right]-\operatorname{Tr}\left[\rho_{3}^{2}\right]\right)=3 C^{2}(\rho)=3 \tau_{3}(\rho),
\end{aligned}
$$


where we have used the similar analysis in $[67,113]$ to obtain the equalities $\sum_{\alpha, \beta} \operatorname{Tr}\left[\rho_{12}\left(\tilde{\rho}_{12}\right)_{\alpha \beta}\right]=1-\operatorname{Tr}\left[\rho_{1}^{2}\right]-\operatorname{Tr}\left[\rho_{2}^{2}\right]+\operatorname{Tr}\left[\rho_{3}^{2}\right], \sum_{\alpha, \beta} \operatorname{Tr}\left[\rho_{13}\left(\tilde{\rho}_{13}\right)_{\alpha \beta}\right]=1-\operatorname{Tr}\left[\rho_{1}^{2}\right]+\operatorname{Tr}\left[\rho_{2}^{2}\right]-\operatorname{Tr}\left[\rho_{3}^{2}\right]$, and $\sum_{\alpha, \beta} \operatorname{Tr}\left[\rho_{23}\left(\tilde{\rho}_{23}\right)_{\alpha \beta}\right]=1+\operatorname{Tr}\left[\rho_{1}^{2}\right]-\operatorname{Tr}\left[\rho_{2}^{2}\right]-\operatorname{Tr}\left[\rho_{3}^{2}\right]$. The last equality is due to the fact that $\rho$ is a pure state.

In fact, the bipartite entanglement inside a tripartite state is useful for distilling maximally entangled states. Assume that there are two of the qualities $\left\{\tau\left(\rho_{12}\right), \tau\left(\rho_{13}\right), \tau\left(\rho_{23}\right)\right\}$ larger than zero; say $\tau\left(\rho_{12}\right)>0$ and $\tau\left(\rho_{13}\right)>0$. According to [67], one can distill two maximal entangled states $\left|\psi_{12}\right\rangle$ and $\left|\psi_{13}\right\rangle$ which belong to $\mathscr{l}_{1} \otimes \mathscr{l}_{2}$ and $\mathscr{l}_{1} \otimes \mathscr{l}_{3}$, respectively. In terms of the result in [114], one can use them to produce a GHZ state.

\subsubsection{Estimation of Multipartite Entanglement}

For a pure $N$-partite quantum state $|\psi\rangle \in \mathfrak{l}_{1} \otimes \mathfrak{l}_{2} \otimes \cdots \otimes \mathfrak{l}_{N}, \operatorname{dim} \mathfrak{l}_{i}=d_{i}, i=1, \ldots, N$, the concurrence of bipartite decomposition between subsystems $12 \cdots M$ and $M+1 \cdots N$ is defined by

$$
C_{2}(|\psi\rangle)=\sqrt{2\left(1-\operatorname{Tr}\left[\rho_{12 \cdots M}^{2}\right]\right)}
$$

where $\rho_{12 \cdots M}^{2}=\operatorname{Tr}_{M+1 \cdots N}[|\psi\rangle\langle\psi|]$ is the reduced density matrix of $\rho=|\psi\rangle\langle\psi|$ by tracing over subsystems $M+1 \cdots N$. On the other hand, the concurrence of $|\psi\rangle$ is defined by (3.9).

For a mixed multipartite quantum state $\rho=\sum_{i} p_{i}\left|\psi_{i}\right\rangle\left\langle\psi_{i}\right| \in \mathscr{l}_{1} \otimes \mathscr{l}_{2} \otimes \cdots \otimes \mathscr{l}_{N}$, the corresponding concurrences of (3.35) and (3.9) are then given by the convex roof

$$
C_{2}(\rho)=\min _{\left.\left\{p_{i}, \psi_{i}\right\}\right\rangle} \sum_{i} p_{i} C_{2}\left(\left|\psi_{i}\right\rangle\left\langle\psi_{i}\right|\right),
$$

and (3.12). We now investigate the relation between these two kinds of concurrences.

Lemma 3.5. For a bipartite density matrix $\rho \in \mathfrak{L}_{A} \otimes \mathfrak{l}_{B}$, one has

$$
1-\operatorname{Tr}\left[\rho^{2}\right] \leq 1-\operatorname{Tr}\left[\rho_{A}^{2}\right]+1-\operatorname{Tr}\left[\rho_{B}^{2}\right]
$$

where $\rho_{A / B}=\operatorname{Tr}_{B / A}[\rho]$ are the reduced density matrices of $\rho$. 
Proof. Let $\rho=\sum_{i j} \lambda_{i j}|i j\rangle\langle i j|$ be the spectral decomposition, where $\lambda_{i j} \geq 0, \sum_{i j} \lambda_{i j}=1$. Then $\rho_{1}=\sum_{i j} \lambda_{i j}|i\rangle\left\langle i\left|, \rho_{2}=\sum_{i j} \lambda_{i j}\right| j\right\rangle\langle j|$. Therefore

$$
\begin{aligned}
1-\operatorname{Tr} & {\left[\rho_{A}^{2}\right]+1-\operatorname{Tr}\left[\rho_{B}^{2}\right]-1+\operatorname{Tr}\left[\rho^{2}\right] } \\
& =1-\operatorname{Tr}\left[\rho_{A}^{2}\right]-\operatorname{Tr}\left[\rho_{B}^{2}\right]+\operatorname{Tr}\left[\rho^{2}\right] \\
& =\left(\sum_{i j} \lambda_{i j}\right)^{2}-\sum_{i, j, j^{\prime}} \lambda_{i j} \lambda_{i j^{\prime}}-\sum_{i, i^{\prime}, j} \lambda_{i j} \lambda_{i^{\prime} j}+\sum_{i j} \lambda_{i j}^{2} \\
& =\left(\sum_{i=i^{\prime}, j=j^{\prime}} \lambda_{i j}^{2}+\sum_{i=i^{\prime}, j \neq j^{\prime}} \lambda_{i j} \lambda_{i j^{\prime}}+\sum_{i \neq i^{\prime}, j=j^{\prime}} \lambda_{i j} \lambda_{i^{\prime} j}+\sum_{i \neq i^{\prime}, j \neq j^{\prime}} \lambda_{i j} \lambda_{i^{\prime} j^{\prime}}\right) \\
& =\sum_{i \neq i^{\prime}, j \neq j^{\prime}} \lambda_{i j} \lambda_{i^{\prime} j^{\prime}} \geq 0 .
\end{aligned}
$$

This lemma can be also derived in another way $[46,115]$.

Theorem 3.6. For a multipartite quantum state $\rho \in \mathfrak{l}_{1} \otimes \mathfrak{L}_{2} \otimes \cdots \otimes \mathfrak{l}_{N}$ with $N \geq 3$, the following inequality holds [116]:

$$
C_{N}(\rho) \geq \max 2^{(3-N) / 2} C_{2}(\rho),
$$

where the maximum is taken over all kinds of bipartite concurrence.

Proof. Without loss of generality, we suppose that the maximal bipartite concurrence is attained between subsystems $12 \cdots M$ and $(M+1) \cdots N$.

For a pure multipartite state $|\psi\rangle \in \mathscr{L}_{1} \otimes \mathfrak{L}_{2} \otimes \cdots \otimes \mathscr{\ell}_{N}, \operatorname{Tr}\left[\rho_{12 \cdots M}^{2}\right]=\operatorname{Tr}\left[\rho_{(M+1) \cdots N}^{2}\right]$. From (3.37) we have

$$
\begin{aligned}
C_{N}^{2}(|\psi\rangle\langle\psi|) & =2^{2-N}\left(\left(2^{N}-2\right)-\sum_{\alpha} \operatorname{Tr}\left[\rho_{\alpha}^{2}\right]\right) \geq 2^{3-N}\left(N-\sum_{k=1}^{N} \operatorname{Tr}\left[\rho_{k}^{2}\right]\right) \\
& \geq 2^{3-N}\left(1-\operatorname{Tr}\left[\rho_{12 \cdots M}^{2}\right]+1-\operatorname{Tr}\left[\rho_{(M+1) \cdots N}^{2}\right]\right) \\
& =2^{3-N} * 2\left(1-\operatorname{Tr}\left[\rho_{12 \cdots M}^{2}\right]\right)=2^{3-N} C_{2}^{2}(|\psi\rangle\langle\psi|),
\end{aligned}
$$

that is, $C_{N}(|\psi\rangle\langle\psi|) \geq 2^{(3-N) / 2} C_{2}(|\psi\rangle\langle\psi|)$. 
Let $\rho=\sum_{i} p_{i}\left|\psi_{i}\right\rangle\left\langle\psi_{i}\right|$ attain the minimal decomposition of the multipartite concurrence. One has

$$
\begin{aligned}
C_{N}(\rho) & =\sum_{i} p_{i} C_{N}\left(\left|\psi_{i}\right\rangle\left\langle\psi_{i}\right|\right) \geq 2^{(3-N) / 2} \sum_{i} p_{i} C_{2}\left(\left|\psi_{i}\right\rangle\left\langle\psi_{i}\right|\right) \\
& \geq 2^{(3-N) / 2} \min _{\left\{p_{i}, \mid \psi_{i}\right\}} \sum_{i} p_{i} C_{2}\left(\left|\psi_{i}\right\rangle\left\langle\psi_{i}\right|\right)=2^{(3-N) / 2} C_{2}(\rho) .
\end{aligned}
$$

Corollary 3.7. For a tripartite quantum state $\rho \in \mathscr{d}_{1} \otimes \mathfrak{d}_{2} \otimes \mathfrak{d}_{3}$, the following inequality holds:

$$
C_{3}(\rho) \geq \max C_{2}(\rho)
$$

where the maximum is taken over all kinds of bipartite concurrence.

In $[46,64]$, from the separability criteria related to local uncertainty relation, covariance matrix, and correlation matrix, the following lower bounds for bipartite concurrence are obtained:

$$
\begin{gathered}
C_{2}(\rho) \geq \frac{2\|C(\rho)\|-\left(1-\operatorname{Tr}\left[\rho_{A}^{2}\right]\right)-\left(1-\operatorname{Tr}\left[\rho_{B}^{2}\right]\right)}{\sqrt{2 d_{A}\left(d_{A}-1\right)}}, \\
C_{2}(\rho) \geq \sqrt{\frac{8}{d_{A}^{3} d_{B}^{2}\left(d_{A}-1\right)}}\left(\|T(\rho)\|-\frac{\sqrt{d_{A} d_{B}\left(d_{A}-1\right)\left(d_{B}-1\right)}}{2}\right),
\end{gathered}
$$

where the entries of the matrix $C, C_{i j}=\left\langle\lambda_{i}^{A} \otimes \lambda_{j}^{B}\right\rangle-\left\langle\lambda_{i}^{A} \otimes I_{d_{B}}\right\rangle\left\langle I_{d_{A}} \otimes \lambda_{j}^{B}\right\rangle, T_{i j}=d_{A} d_{B} / 2\left\langle\lambda_{i}^{A} \otimes \lambda_{j}^{B}\right\rangle$, $\lambda_{k}^{A / B}$ stand for the normalized generator of $S U\left(d_{A} / d_{B}\right)$, that is, $\operatorname{Tr}\left[\lambda_{k}^{A / B} \lambda_{l}^{A / B}\right]=\delta_{k l}$ and $\langle X\rangle=$ $\operatorname{Tr}[\rho X]$. It is shown that the lower bounds (3.43) and (3.44) are independent of (3.13).

Now we consider a multipartite quantum state $\rho \in \mathscr{L}_{1} \otimes \mathfrak{l}_{2} \otimes \cdots \otimes \mathscr{l}_{N}$ as a bipartite state belonging to $\mathscr{\ell}^{A} \otimes \mathfrak{L}^{B}$ with the dimensions of the subsystems $A$ and $B$ being $d_{A}=$ $d_{s_{1}} d_{s_{2}} \cdots d_{s_{m}}$ and $d_{B}=d_{s_{m+1}} d_{s_{m+2}} \cdots d_{s_{N}}$, respectively. By using Corollary 3.7, (3.13), (3.43), and (3.44), one has the following lower bound.

Theorem 3.8. For any N-partite quantum state $\rho$ [116],

$$
C_{N}(\rho) \geq 2^{(3-N) / 2} \max \left\{B_{1}, B_{2}, B_{3}\right\}
$$

where

$$
\begin{aligned}
& B_{1}=\max _{\{i\}} \sqrt{\frac{2}{M_{i}\left(M_{i}-1\right)}\left[\max \left(\left\|\tau_{A}\left(\rho^{i}\right)\right\|,\left\|R\left(\rho^{i}\right)\right\|\right)-1\right],} \\
& B_{2}=\max _{\{i\}} \frac{2\left\|C\left(\rho^{i}\right)\right\|-\left(1-\operatorname{Tr}\left[\left(\rho_{A}^{i}\right)^{2}\right]\right)-\left(1-\operatorname{Tr}\left[\left(\rho_{B}^{i}\right)^{2}\right]\right)}{\sqrt{2 M_{i}\left(M_{i}-1\right)}}, \\
& B_{3}=\max _{\{i\}} \sqrt{\frac{8}{M_{i}^{3} N_{i}^{2}\left(M_{i}-1\right)}}\left(\left\|T\left(\rho^{i}\right)\right\|-\frac{\sqrt{M_{i} N_{i}\left(M_{i}-1\right)\left(N_{i}-1\right)}}{2}\right),
\end{aligned}
$$


$\rho^{i \prime}$ s are all possible bipartite decompositions of $\rho$, and

$$
\begin{aligned}
& M_{i}=\min \left\{d_{s_{1}} d_{s_{2}} \cdots d_{s_{m}}, d_{s_{m+1}} d_{s_{m+2}} \cdots d_{s_{N}}\right\}, \\
& N_{i}=\max \left\{d_{s_{1}} d_{s_{2}} \cdots d_{s_{m}}, d_{s_{m+1}} d_{s_{m+2}} \cdots d_{s_{N}}\right\} .
\end{aligned}
$$

In $[46,106,107,117]$, it is shown that the upper and lower bounds of multipartite concurrence satisfy

$$
\sqrt{\left(4-2^{3-N}\right) \operatorname{Tr}\left[\rho^{2}\right]-2^{2-N} \sum_{\alpha} \operatorname{Tr}\left[\rho_{\alpha}^{2}\right]} \leq C_{N}(\rho) \leq \sqrt{2^{2-N}\left[\left(2^{N}-2\right)-\sum_{\alpha} \operatorname{Tr}\left[\rho_{\alpha}^{2}\right]\right]} .
$$

In fact one can obtain a more effective upper bound for multipartite concurrence. Let $\rho=\sum_{i} \lambda_{i}\left|\psi_{i}\right\rangle\left\langle\psi_{i}\right| \in \mathscr{l}_{1} \otimes \mathfrak{l}_{2} \otimes \cdots \otimes \mathscr{l}_{N}$, where $\left|\psi_{i}\right\rangle$ 's are the orthogonal pure states and $\sum_{i} \lambda_{i}=1$. We have

$$
C_{N}(\rho)=\min _{\left\{p_{i},\left|\varphi_{i}\right\rangle\right\rangle} \sum_{i} p_{i} C_{N}\left(\left|\varphi_{i}\right\rangle\left\langle\varphi_{i}\right|\right) \leq \sum_{i} \lambda_{i} C_{N}\left(\left|\psi_{i}\right\rangle\left\langle\psi_{i}\right|\right) .
$$

The right side of (3.49) gives a new upper bound of $C_{N}(\rho)$. Since

$$
\begin{aligned}
\sum_{i} \lambda_{i} C_{N}\left(\left|\psi_{i}\right\rangle\left\langle\psi_{i}\right|\right) & =2^{1-N / 2} \sum_{i} \lambda_{i} \sqrt{\left(2^{N}-2\right)-\sum_{\alpha} \operatorname{Tr}\left[\left(\rho_{\alpha}^{i}\right)^{2}\right]} \\
& \leq 2^{1-N / 2} \sqrt{\left(2^{N}-2\right)-\sum_{\alpha} \operatorname{Tr}\left[\sum_{i} \lambda_{i}\left(\rho_{\alpha}^{i}\right)^{2}\right]} \\
& \leq 2^{1-N / 2} \sqrt{\left(2^{N}-2\right)-\sum_{\alpha} \operatorname{Tr}\left[\left(\rho_{\alpha}\right)^{2}\right]}
\end{aligned}
$$

the upper bound obtained in (3.49) is better than that in (3.48).

\subsubsection{Bounds of Concurrence and Tangle}

In [68], a lower bound for tangle defined in (3.8) has been derived as

$$
\tau(\rho) \geq \frac{8}{M N(M+N)}\left(\|T(\rho)\|_{H S}^{2}-\frac{M N(M-1)(N-1)}{4}\right),
$$

where $\|X\|_{H S}=\sqrt{\operatorname{Tr}\left[X X^{\dagger}\right]}$ denotes the Frobenius or Hilbert-Schmidt norm. Experimentally measurable lower and upper bounds for concurrence have been also given by Mintert et al. in $[106,107]$ and Zhang et al. in [46]:

$$
\sqrt{2\left(\operatorname{Tr}\left[\rho^{2}\right]-\operatorname{Tr}\left[\rho_{A}^{2}\right]\right)} \leq C(\rho) \leq \sqrt{2\left(1-\operatorname{Tr}\left[\rho_{A}^{2}\right]\right)} .
$$


Since the convexity of $C^{2}(\rho)$, we have that $\tau(\rho) \geq C^{2}(\rho)$ always holds. For two-qubit quantum systems, tangle $\tau$ is always equal to the square of concurrence $C^{2}[58,113]$, as a decomposition $\left\{p_{i},\left|\psi_{i}\right\rangle\right\}$ achieving the minimum in (3.3) has the property that $C\left(\left|\psi_{i}\right\rangle\right)=$ $C\left(\left|\psi_{j}\right\rangle\right) \forall i, j$. For higher dimensional systems we do not have similar relations. Thus it is meaningful to derive valid upper bound for tangle and lower bound for concurrence.

Theorem 3.9. For any quantum state $\rho \in \mathscr{H}_{A} \otimes \mathfrak{l}_{B}$, one has [118]

$$
\begin{gathered}
\tau(\rho) \leq \min \left\{1-\operatorname{Tr}\left[\rho_{A}^{2}\right], 1-\operatorname{Tr}\left[\rho_{B}^{2}\right]\right\}, \\
C(\rho) \geq \sqrt{\frac{8}{M N(M+N)}}\left(\|T(\rho)\|_{H S}-\frac{\sqrt{M N(M-1)(N-1)}}{2}\right),
\end{gathered}
$$

where $\rho_{A}$ is the reduced matrix of $\rho$, and $T(\rho)$ is the correlation matrix of $\rho$ defined in (3.44).

Proof. We assume that $1-\operatorname{Tr}\left[\rho_{A}^{2}\right] \leq 1-\operatorname{Tr}\left[\rho_{B}^{2}\right]$ for convenience. By the definition of $\tau$, we have that for a pure state $|\psi\rangle, \tau(|\psi\rangle)=2\left(1-\operatorname{Tr}\left[\left(\rho_{A}^{|\psi\rangle}\right)^{2}\right]\right)$. Let $\rho=\sum_{i} p_{i} \rho_{i}$ be the optimal decomposition such that $\tau(\rho)=\sum_{i} p_{i} \tau\left(\rho_{i}\right)$. We get

$$
\tau(\rho)=\sum_{i} p_{i} \tau\left(\rho_{i}\right)=\sum_{i} p_{i} 2\left[1-\operatorname{Tr}\left[\left(\rho_{A}^{\left|\psi_{i}\right\rangle}\right)^{2}\right]\right]=2\left[1-\operatorname{Tr}\left[\sum_{i} p_{i}\left(\rho_{A}^{\left|\psi_{i}\right\rangle}\right)^{2}\right]\right] \leq 2\left[1-\operatorname{Tr}\left[\rho_{A}^{2}\right]\right] .
$$

Note that, for pure state $|\psi\rangle \in \mathscr{l}_{A} \otimes \mathscr{l}_{B}[68]$,

$$
C(|\psi\rangle)=\sqrt{\frac{8}{M N(M+N)}\left(\| T(|\psi\rangle) \|^{2}-\frac{M N(M-1)(N-1)}{4}\right)} .
$$

Using the inequality $\sqrt{a-b} \geq \sqrt{a}-\sqrt{b}$ for any $a \geq b$, we get

$$
C(|\psi\rangle) \geq \sqrt{\frac{8}{M N(M+N)}}\left(\| T(|\psi\rangle) \|_{H S}-\frac{\sqrt{M N(M-1)(N-1)}}{2}\right) .
$$

Now let $\rho=\sum_{i} p_{i} \rho_{i}$ be the optimal decomposition such that $C(\rho)=\sum_{i} p_{i} C\left(\rho_{i}\right)$. We get

$$
\begin{aligned}
C(\rho) & =\sum_{i} p_{i} C\left(\rho_{i}\right) \geq \sum_{i} p_{i} \sqrt{\frac{8}{M N(M+N)}}\left(\left\|T\left(\rho_{i}\right)\right\|_{H S}-\frac{\sqrt{M N(M-1)(N-1)}}{2}\right) \\
& =\sqrt{\frac{8}{M N(M+N)}}\left(\sum_{i} p_{i}\left\|T\left(\rho_{i}\right)\right\|_{H S}-\frac{\sqrt{M N(M-1)(N-1)}}{2}\right) \\
& \geq \sqrt{\frac{8}{M N(M+N)}}\left(\|T(\rho)\|_{H S}-\frac{\sqrt{M N(M-1)(N-1)}}{2}\right),
\end{aligned}
$$

which ends the proof. 
The upper bound (3.53), together with the lower bounds (3.54), (3.43), (3.44), (3.51), and (3.52), can allow for estimations of entanglement for arbitrary quantum states. Moreover, since the upper bound is exactly the value of tangle for pure states, the upper bound can be a good estimation when the state is very weakly mixed.

\subsection{Concurrence and Tangle of Two Entangled States Are Strictly Larger Than Those of One}

In this subsection we show that although bound entangled states cannot be distilled, the concurrence and tangle of two entangled states will be always strictly larger than those of one, even if the two entangled states are both bound entangled.

Let $\rho=\sum_{i j k l} \rho_{i j, k l}|i j\rangle\langle k l| \in \mathscr{H}_{A} \otimes \mathscr{H}_{B}$ and $\sigma=\sum_{i^{\prime} j^{\prime} k^{\prime} l^{\prime}} \sigma_{i^{\prime} j^{\prime}, k^{\prime} l^{\prime}}\left|i^{\prime} j^{\prime}\right\rangle\left\langle k^{\prime} l^{\prime}\right| \in \mathscr{H}_{A^{\prime}} \otimes$ $\mathscr{H}_{B^{\prime}}$ be two quantum states shared by subsystems $A A^{\prime}$ and $B B^{\prime}$. We use $\rho \otimes \sigma=$ $\sum_{i j k l, i^{\prime} j^{\prime} k^{\prime} l^{\prime}} \rho_{i j, k l} \sigma_{i^{\prime} j^{\prime}, k^{\prime} l^{\prime}}\left|i i^{\prime}\right\rangle_{A A^{\prime}}\left\langle k k^{\prime}|\otimes| j j^{\prime}\right\rangle_{B B^{\prime}}\left\langle l l^{\prime}\right|$ to denote the state of the whole system.

Lemma 3.10. For pure states $|\psi\rangle \in \mathscr{l}_{A} \otimes \mathscr{l}_{B}$ and $|\varphi\rangle \in \mathscr{l}_{A^{\prime}} \otimes \mathscr{l}_{B^{\prime}}$, the inequalities

$$
\begin{gathered}
C(|\psi\rangle \otimes|\varphi\rangle) \geq \max \{C(|\psi\rangle), C(|\varphi\rangle)\}, \\
\tau(|\psi\rangle \otimes|\varphi\rangle) \geq \max \{\tau(|\psi\rangle), \tau(|\varphi\rangle)\}
\end{gathered}
$$

always hold, and " $="$ in the two inequalities hold if and only if at least one of $\{|\psi\rangle,|\varphi\rangle\}$ is separable.

Proof. Without loss of generality we assume that $C(|\psi\rangle) \geq C(|\varphi\rangle)$. First note that

$$
\rho_{A A^{\prime}}^{|\psi\rangle \otimes\rangle}=\rho_{A}^{|\psi\rangle} \otimes \rho_{A^{\prime}}^{|\varphi\rangle}
$$

Let $\rho_{A}^{|\psi\rangle}=\sum_{i} \lambda_{i}|i\rangle\langle i|$ and $\rho_{A^{\prime}}^{|\varphi\rangle}=\sum_{j} \pi_{j}|j\rangle\langle j|$ be the spectral decomposition of $\rho_{A}^{|\psi\rangle}$ and $\rho_{A^{\prime}}^{|\varphi\rangle}$, with $\sum_{i} \lambda_{i}=1$ and $\sum_{j} \pi_{j}=1$, respectively. By using (3.61) one obtains that

$$
\operatorname{Tr}\left[\left(\rho_{A A^{\prime}}^{|\psi\rangle \otimes|\varphi\rangle}\right)^{2}\right]=\sum \lambda_{i} \pi_{j} \lambda_{i^{\prime}} \pi_{j^{\prime}}|i j\rangle\left\langle i j \mid i^{\prime} j^{\prime}\right\rangle\left\langle i^{\prime} j^{\prime}\right|=\sum \lambda_{i}^{2} \pi_{j}^{2}
$$

while

$$
\operatorname{Tr}\left[\left(\rho_{A}^{|\psi\rangle}\right)^{2}\right]=\sum_{i} \lambda_{i}^{2}
$$

Now using the definition of concurrence and the normalization conditions of $\lambda_{i}$ and $\pi_{j}$, one immediately gets

$$
C(|\psi\rangle \otimes|\varphi\rangle)=\sqrt{2\left(1-\operatorname{Tr}\left[\left(\rho_{A A^{\prime}}^{|\psi\rangle}\right)^{\prime}\right]\right)} \geq \sqrt{2\left(1-\operatorname{Tr}\left[\left(\rho_{A}^{|\psi\rangle}\right)^{2}\right]\right)}=C(|\psi\rangle)
$$


If one of $\{|\psi\rangle,|\varphi\rangle\}$ is separable, say $|\varphi\rangle$, then the rank of $\rho_{A^{\prime}}^{|\varphi\rangle}$ must be one, which means that there is only one item in the spectral decomposition in $\rho_{A^{\prime}}^{|\varphi\rangle}$. Using the normalization condition of $\pi_{j}$, we obtain $\operatorname{Tr}\left[\left(\rho_{A A^{\prime}}^{|\psi\rangle|\varphi\rangle}\right)^{2}\right]=\operatorname{Tr}\left[\left(\rho_{A}^{|\psi\rangle}\right)^{2}\right]$. Then inequality (3.64) becomes an equality.

On the other hand, if both $|\psi\rangle$ and $|\varphi\rangle$ are entangled (not separable), then there must be at least two items in the decomposition of their reduced density matrices $\rho_{A}^{|\psi\rangle}$ and $\rho_{A^{\prime}}^{|\varphi\rangle}$, which means that $\operatorname{Tr}\left[\left(\rho_{A A^{\prime}}^{|\psi\rangle|\varphi\rangle}\right)^{2}\right]$ is strictly larger than $\operatorname{Tr}\left[\left(\rho_{A}^{|\psi\rangle}\right)^{2}\right]$.

The inequality (3.60) also holds because, for pure quantum state $\rho, \tau(\rho)=C^{2}(\rho)$.

From the lemma, we have, for mixed states the following.

Theorem 3.11. For any quantum states $\rho \in \mathscr{l}_{A} \otimes \mathfrak{l}_{B}$ and $\sigma \in \mathscr{d}_{A^{\prime}} \otimes \mathfrak{l}_{B^{\prime}}$, the inequalities

$$
\begin{gathered}
C(\rho \otimes \sigma) \geq \max \{C(\rho), C(\sigma)\}, \\
\tau(\rho \otimes \sigma) \geq \max \{\tau(\rho), \tau(\sigma)\}
\end{gathered}
$$

always hold, and the " $="$ in the two inequalities hold if and only if at least one of $\{\rho, \sigma\}$ is separable, that is, if both $\rho$ and $\sigma$ are entangled (even if bound entangled), then $C(\rho \otimes \sigma)>\max \{C(\rho), C(\sigma)\}$ and $\tau(\rho \otimes \sigma)>\max \{\tau(\rho), \tau(\sigma)\}$ always hold [118].

Proof. We still assume that $C(\rho) \geq C(\sigma)$ for convenience. Let $\rho=\sum_{i} p_{i} \rho_{i}$ and $\sigma=\sum_{j} q_{j} \sigma_{j}$ be the optimal decomposition such that $C(\rho \otimes \sigma)=\sum_{i} p_{i} q_{j} C\left(\rho_{i} \otimes \sigma_{j}\right)$. By using the inequality obtained in Lemma 3.10, we have

$$
C(\rho \otimes \sigma)=\sum_{i} p_{i} q_{j} C\left(\rho_{i} \otimes \sigma_{j}\right) \geq \sum_{i} p_{i} q_{j} C\left(\rho_{i}\right)=\sum_{i} p_{i} C\left(\rho_{i}\right) \geq C(\rho)
$$

Case 1. Now let one of $\{\rho, \sigma\}$ be separable, say $\sigma$, with ensemble representation $\sigma=\sum_{j} q_{j} \sigma_{j}$, where $\sum_{j} q_{j}=1$, and $\sigma_{j}$ is the density matrix of separable pure state. Suppose that $\rho=\sum_{i} p_{i} \rho_{i}$ is the optimal decomposition of $\rho$ such that $C(\rho)=\sum_{i} p_{i} C\left(\rho_{i}\right)$. Using Lemma 3.10, we have

$$
C(\rho \otimes \sigma) \leq \sum_{i} p_{i} q_{j} C\left(\rho_{i} \otimes \sigma_{j}\right)=\sum_{i} p_{i} q_{j} C\left(\rho_{i}\right)=\sum_{i} p_{i} C\left(\rho_{i}\right)=C(\rho)
$$

The inequalities (3.66) and (3.67) show that if $\sigma$ is separable, then $C(\rho \otimes \sigma)=C(\rho)$.

Case 2. If both $\rho$ and $\sigma$ are inseparable, that is, there is at least one pure state in the ensemble decomposition of $\rho$ (and $\sigma$, resp.), using Lemma 3.10, then we have

$$
C(\rho \otimes \sigma)=\sum_{i} p_{i} q_{j} C\left(\rho_{i} \otimes \sigma_{j}\right)>\sum_{i} p_{i} q_{j} C\left(\rho_{i}\right)=\sum_{i} p_{i} C\left(\rho_{i}\right) \geq C(\rho)
$$

The inequality for tangle $\tau$ can be proved in a similar way.

Remark 3.12. In [119] it is shown that any entangled state $\rho$ can enhance the teleportation power of another state $\sigma$. This holds even if the state $\rho$ is bound entangled. But if $\rho$ is bound entangled, then the corresponding $\sigma$ must be free entangled (distillable). By Theorem 3.11, 
we can see that even if two entangled quantum states $\rho$ and $\sigma$ are bound entangled, their concurrence and tangle are strictly larger than those of one state.

\subsection{Subadditivity of Concurrence and Tangle}

We now give a proof of the subadditivity of concurrence and tangle, which illustrates that concurrence and tangle may be proper entanglement measurements.

Theorem 3.13. Let $\rho$ and $\sigma$ be quantum states in $\mathfrak{d}_{A} \otimes \mathfrak{d}_{B}$, the one has [118]

$$
C(\rho \otimes \sigma) \leq C(\rho)+C(\sigma), \quad \tau(\rho \otimes \sigma) \leq \tau(\rho)+\tau(\sigma) .
$$

Proof. We first prove that the theorem holds for pure states, that is, for $|\psi\rangle$ and $|\phi\rangle$ in $\mathscr{L}_{A} \otimes \mathscr{L}_{B}$ :

$$
C(|\psi\rangle \otimes|\phi\rangle) \leq C(|\psi\rangle)+C(|\phi\rangle), \quad \tau(|\psi\rangle \otimes|\phi\rangle) \leq \tau(|\psi\rangle)+\tau(|\phi\rangle) .
$$

Assume that $\rho_{A}^{|\psi\rangle}=\sum_{i} \lambda_{i}|i\rangle\langle i|$ and $\rho_{A}^{|\phi\rangle}=\sum_{j} \pi_{j}|j\rangle\langle j|$ are the spectral decomposition of the reduced matrices $\rho_{A}^{|\psi\rangle}$ and $\rho_{A}^{|\phi\rangle}$. One has

$$
\begin{aligned}
\frac{1}{2}[C(|\psi\rangle)+C(|\phi\rangle)]^{2} & \geq 1-\operatorname{Tr}\left[\left(\rho_{A}^{|\psi\rangle}\right)^{2}\right]+1-\operatorname{Tr}\left[\left(\rho_{A}^{|\phi\rangle}\right)^{2}\right] \\
& =1-\sum_{i} \lambda_{i}^{2}+1-\sum_{j} \pi_{j}^{2} \geq 1-\sum_{i j} \lambda_{i}^{2} \pi_{j}^{2}=\frac{1}{2} C^{2}(|\psi\rangle \otimes|\phi\rangle) .
\end{aligned}
$$

Now we prove that (3.69) holds for any mixed-quantum states $\rho$ and $\sigma$. Let $\rho=\sum_{i} p_{i} \rho_{i}$ and $\sigma=\sum_{j} q_{j} \sigma_{j}$ be the optimal decomposition such that $C(\rho)=\sum_{i} p_{i} C\left(\rho_{i}\right)$ and $C(\sigma)=$ $\sum_{j} q_{j} C\left(\sigma_{j}\right)$. We have

$$
C(\rho)+C(\sigma)=\sum_{i j} p_{i} q_{j}\left[C\left(\rho_{i}\right)+C\left(\sigma_{j}\right)\right] \geq \sum_{i j} p_{i} q_{j} C\left(\rho_{i} \otimes \sigma_{j}\right) \geq C(\rho \otimes \sigma) .
$$

The inequality for $\tau$ can be derived in a similar way.

\section{Fidelity of Teleportation and Distillation of Entanglement}

Quantum teleportation is an important subject in quantum information processing. In terms of a classical communication channel and a quantum resource (a nonlocal entangled state like an EPR pair of particles), the teleportation protocol gives ways to transmit an unknown quantum state from a sender traditionally named "Alice" to a receiver "Bob" who are spatially separated. These teleportation processes can be viewed as quantum channels. The nature of a quantum channel is determined by the particular protocol and the state used as a teleportation resource. The standard teleportation protocol $T_{0}$ proposed by Bennett et al. in 1993 uses Bell measurements and Pauli rotations. When the maximally entangled pure state $|\phi\rangle=(1 / \sqrt{n}) \sum_{i=0}^{n-1}|i i\rangle$ is used as the quantum resource, it provides an ideal 
noiseless quantum channel $\Lambda_{T_{0}}^{(|\phi\rangle\langle\phi|)}(\rho)=\rho$. However in realistic situation, instead of the pure maximally entangled states, Alice and Bob usually share a mixed entangled state due to the decoherence. Teleportation using mixed state as an entangled resource is, in general, equivalent to having a noisy quantum channel. An explicit expression for the output state of the quantum channel associated with the standard teleportation protocol $T_{0}$ with an arbitrary mixed-state resource has been obtained $[120,121]$.

It turns out that, by local quantum operations (including collective actions over all members of pairs in each lab) and classical communication (LOCC) between Alice and Bob, it is possible to obtain a number of pairs in nearly maximally entangled state $\left|\psi_{+}\right\rangle$from many pairs of nonmaximally entangled states. Such a procedure proposed in [73-77] is called distillation. In [73] the authors give operational protocol to distill an entangled two-qubit state whose single fraction $F$, defined by $F(\rho)=\left\langle\psi_{+}|\rho| \psi_{+}\right\rangle$, is larger than $1 / 2$. The protocol is then generalized in [77] to distill any $d$-dimensional bipartite entangled quantum states with $F(\rho)>1 / d$. It is shown that a quantum state $\rho$ violating the reduction criterion can always be distilled. For such states, if their single fraction of entanglement $F(\rho)=\left\langle\psi_{+}|\rho| \psi_{+}\right\rangle$is greater than $1 / d$, one can distill these states directly by using the generalized distillation protocol, otherwise a proper filtering operation has to be used at first to transform $\rho$ to another state $\rho^{\prime}$ so that $F\left(\rho^{\prime}\right)>1 / d$.

\subsection{Fidelity of Quantum Teleportation}

Let $\mathscr{t}$ be a $d$-dimensional complex vector space with computational basis $|i\rangle, i=1, \ldots, d$. The fully entangled fraction (FEF) of a density matrix $\rho \in \mathscr{l} \otimes \mathscr{d}$ is defined by

$$
\mathcal{F}(\rho)=\max _{U}\left\langle\psi_{+}\left|\left(I \otimes U^{\dagger}\right) \rho(I \otimes U)\right| \psi_{+}\right\rangle
$$

under all unitary transformations $U$, where $\left|\psi_{+}\right\rangle=(1 / \sqrt{d}) \sum_{i=1}^{d}|i i\rangle$ is the maximally entangled state and $I$ is the corresponding identity matrix.

In $[8,9]$, the authors give an optimal teleportation protocol by using a mixed entangled quantum state. The optimal teleportation fidelity is given by

$$
f_{\max }(\rho)=\frac{d \mathcal{F}(\rho)}{d+1}+\frac{1}{d+1}
$$

which solely depends on the FEF of the entangled resource state $\rho$.

In fact the fully entangled fraction is tightly related to many quantum information processing such as dense coding [10], teleportation [5-7], entanglement swapping [14-18], and quantum cryptography (Bell inequalities) [11-13]. As the optimal fidelity of teleportation is given by FEF $[8,9]$, experimentally measurement of FEF can be also used to determine the entanglement of the nonlocal source used in teleportation. Thus an analytic formula for FEF is of great importance. In [122] an elegant formula of FEF for two-qubit system is derived analytically by using the method of Lagrange multipliers. For high-dimensional quantum states the analytical computation of FEF remains formidable and less results have been known. In the following we give an estimation on the values of FEF by giving some upper bounds of FEF. 
Let $\lambda_{i}, i=1, \ldots, d^{2}-1$, be the generators of the $S U(d)$ algebra. A bipartite state $\rho \in$ $\mathscr{H} \otimes \mathscr{H}$ can be expressed as

$$
\rho=\frac{1}{d^{2}} I \otimes I+\frac{1}{d} \sum_{i=1}^{d^{2}-1} r_{i}(\rho) \lambda_{i} \otimes I+\frac{1}{d} \sum_{j=1}^{d^{2}-1} s_{j}(\rho) I \otimes \lambda_{j}+\sum_{i, j=1}^{d^{2}-1} m_{i j}(\rho) \lambda_{i} \otimes \lambda_{j},
$$

where $r_{i}(\rho)=(1 / 2) \operatorname{Tr}\left[\rho \lambda_{i}(1) \otimes I\right], s_{j}(\rho)=(1 / 2) \operatorname{Tr}\left[\rho I \otimes \lambda_{j}(2)\right]$, and $m_{i j}(\rho)=(1 / 4) \operatorname{Tr}\left[\rho \lambda_{i}(1) \otimes\right.$ $\left.\lambda_{j}(2)\right]$. Let $M(\rho)$ denote the correlation matrix with entries $m_{i j}(\rho)$.

Theorem 4.1. For any $\rho \in \mathscr{\ell} \otimes \mathfrak{d}$, the fully entangled fraction $\mathcal{F}(\rho)$ satisfies [123]

$$
\mathcal{F}(\rho) \leq \frac{1}{d^{2}}+4\left\|M^{T}(\rho) M\left(P_{+}\right)\right\|_{K F^{\prime}}
$$

where $M^{T}$ stands for the transpose of $M$ and $\|M\|_{K F}=\operatorname{Tr}\left[\sqrt{M M^{\dagger}}\right]$ is the Ky Fan norm of $M$.

Proof. First, we note that

$$
P_{+}=\frac{1}{d^{2}} I \otimes I+\sum_{i, j=1}^{d^{2}-1} m_{i j}\left(P_{+}\right) \lambda_{i} \otimes \lambda_{j}
$$

where $m_{i j}\left(P_{+}\right)=(1 / 4) \operatorname{Tr}\left[P_{+} \lambda_{i} \otimes \lambda_{j}\right]$. By definition (4.1), one obtains

$$
\begin{aligned}
\mathcal{F}(\rho) & =\max _{U}\left\langle\psi_{+}\left|\left(I \otimes U^{\dagger}\right) \rho(I \otimes U)\right| \psi_{+}\right\rangle=\max _{U} \operatorname{Tr}\left[\rho(I \otimes U) P_{+}\left(I \otimes U^{\dagger}\right)\right] \\
& =\max _{U}\left\{\frac{1}{d^{2}} \operatorname{Tr}[\rho]+\sum_{i, j=1}^{d^{2}-1} m_{i j}\left(P_{+}\right) \operatorname{Tr}\left[\rho \lambda_{i} \otimes U \lambda_{j} U^{\dagger}\right]\right\} .
\end{aligned}
$$

Since $U \lambda_{i} U^{\dagger}$ is a traceless Hermitian operator, it can be expanded according to the $S U(d)$ generators as

$$
U \lambda_{i} U^{\dagger}=\sum_{j=1}^{d^{2}-1} \frac{1}{2} \operatorname{Tr}\left[U \lambda_{i} U^{\dagger} \lambda_{j}\right] \lambda_{j} \equiv \sum_{j=1}^{d^{2}-1} O_{i j} \lambda_{j}
$$

Entries $O_{i j}$ define a real $\left(d^{2}-1\right) \times\left(d^{2}-1\right)$ matrix $O$. From the completeness relation of $S U(d)$ generators

$$
\sum_{j=1}^{d^{2}-1}\left(\lambda_{j}\right)_{k i}\left(\lambda_{j}\right)_{m n}=2 \delta_{i m} \delta_{k n}-\frac{2}{d} \delta_{k i} \delta_{m n}
$$


one can show that $O$ is an orthonormal matrix. Using (4.7), we have

$$
\begin{aligned}
\mathcal{F}(\rho) & \leq \frac{1}{d^{2}}+\max _{O} \sum_{i, j, k} m_{i j}\left(P_{+}\right) O_{j k} \operatorname{Tr}\left[\rho \lambda_{i} \otimes \lambda_{k}\right] \\
& =\frac{1}{d^{2}}+4 \max _{O} \sum_{i, j, k} m_{i j}\left(P_{+}\right) O_{j k} m_{i k}(\rho)=\frac{1}{d^{2}}+4 \max _{O} \operatorname{Tr}\left[M(\rho)^{T} M\left(P_{+}\right) O\right] \\
& =\frac{1}{d^{2}}+4\left\|M(\rho)^{T} M\left(P_{+}\right)\right\|_{K F} .
\end{aligned}
$$

For the case $d=2$, we can get an exact result from (4.4).

Corollary 4.2. For two-qubit system, one has

$$
\mathcal{F}(\rho)=\frac{1}{4}+4\left\|M(\rho)^{T} M\left(P_{+}\right)\right\|_{K F^{\prime}}
$$

that is, the upper bound derived in Theorem 4.1 is exactly the FEF.

Proof. We have shown in (4.7) that, given an arbitrary unitary $U$, one can always obtain an orthonormal matrix $O$. Now we show that in two-qubit case, for any $3 \times 3$ orthonormal matrix $O$, there always exits $2 \times 2$ unitary matrix $U$ such that (4.7) holds.

For any vector $\mathbf{t}=\left\{t_{1}, t_{2}, t_{3}\right\}$ with unit norm, define an operator $X \equiv \sum_{i=1}^{3} t_{i} \sigma_{i}$, where $\sigma_{i}{ }^{\prime}$ s are Pauli matrices. Given an orthonormal matrix $O$, one obtains a new operator $X^{\prime} \equiv$ $\sum_{i=1}^{3} t_{i}^{\prime} \sigma_{i}=\sum_{i, j=1}^{3} O_{i j} t_{j} \sigma_{i}$.

$X$ and $X^{\prime}$ are both Hermitian traceless matrices. Their eigenvalues are given by the norms of the vectors $\mathbf{t}$ and $\mathbf{t}^{\prime}=\left\{t_{1}^{\prime}, t_{2}^{\prime}, t_{3}^{\prime}\right\}$, respectively. As the norms are invariant under orthonormal transformations $O$, they have the same eigenvalues: $\pm \sqrt{t_{1}^{2}+t_{2}^{2}+t_{3}^{2}}$. Thus there must be a unitary matrix $U$ such that $X^{\prime}=U X U^{\dagger}$. Hence the inequality in the proof of Theorem 4.1 becomes an equality. The upper bound (4.4) then becomes exact at this situation, which is in accord with the result in [122].

Remark 4.3. The upper bound of FEF (4.4) and the FEF (4.10) depend on the correlation matrices $M(\rho)$ and $M\left(P_{+}\right)$. They can be calculated directly according to a given set of $S U(d)$ generators $\lambda_{i}, i=1, \ldots, d^{2}-1$. As an example, for $d=3$, if we choose

$$
\begin{aligned}
& \lambda_{1}=\left(\begin{array}{ccc}
1 & 0 & 0 \\
0 & -1 & 0 \\
0 & 0 & 0
\end{array}\right), \quad \lambda_{2}=\left(\begin{array}{ccc}
\frac{1}{\sqrt{3}} & 0 & 0 \\
0 & \frac{1}{\sqrt{3}} & 0 \\
0 & 0 & -\frac{2}{\sqrt{3}}
\end{array}\right), \quad \lambda_{3}=\left(\begin{array}{lll}
0 & 1 & 0 \\
1 & 0 & 0 \\
0 & 0 & 0
\end{array}\right), \quad \lambda_{4}=\left(\begin{array}{lll}
0 & 0 & 1 \\
0 & 0 & 0 \\
1 & 0 & 0
\end{array}\right), \\
& \lambda_{5}=\left(\begin{array}{ccc}
0 & 0 & 0 \\
0 & 0 & 1 \\
0 & 1 & 0
\end{array}\right), \quad \lambda_{6}=\left(\begin{array}{ccc}
0 & i & 0 \\
-i & 0 & 0 \\
0 & 0 & 0
\end{array}\right), \quad \lambda_{7}=\left(\begin{array}{ccc}
0 & 0 & i \\
0 & 0 & 0 \\
-i & 0 & 0
\end{array}\right), \quad \lambda_{8}=\left(\begin{array}{ccc}
0 & 0 & 0 \\
0 & 0 & i \\
0 & -i & 0
\end{array}\right),
\end{aligned}
$$


then we have

$$
M\left(P_{+}\right)=\operatorname{Diag}\left\{\frac{1}{6}, \frac{1}{6}, \frac{1}{6}, \frac{1}{6}, \frac{1}{6},-\frac{1}{6},-\frac{1}{6},-\frac{1}{6}\right\} .
$$

Nevertheless the FEF and its upper bound do not depend on the choice of the $S U(d)$ generators.

The usefulness of the bound depends on detailed states. In the following we give two new upper bounds, which is different from Theorem 4.1. These bounds work for different states.

Let $h$ and $g$ be $n \times n$ matrices such that $h|j\rangle=|(j+1) \bmod n\rangle, g|j\rangle=\omega^{j}|j\rangle$, with $\omega=\exp \{-2 i \pi / n\}$. We can introduce $n^{2}$ linear-independent $n \times n$ matrices $U_{s t}=h^{t} g^{s}$, which satisfy

$$
U_{s t} U_{s^{\prime} t^{\prime}}=\omega^{s t^{\prime}-t s^{\prime}} U_{s^{\prime} t^{\prime}} U_{s t}, \quad \operatorname{Tr}\left[U_{s t}\right]=n \delta_{s 0} \delta_{t 0} .
$$

One can also check that $\left\{U_{s t}\right\}$ satisfy the condition of bases of the unitary operators in the sense of [124], that is,

$$
\operatorname{Tr}\left[U_{s t} U_{s^{\prime} t^{\prime}}^{+}\right]=n \delta_{t t^{\prime}} \delta_{s s^{\prime}}, \quad U_{s t} U_{s t}^{+}=I_{n \times n},
$$

where $I_{n \times n}$ is the $n \times n$ identity matrix. $\left\{U_{s t}\right\}$ form a complete basis of $n \times n$ matrices, namely, for any $n \times n$ matrix $W, W$ can be expressed as

$$
W=\frac{1}{n} \sum_{s, t} \operatorname{Tr}\left[U_{s t}^{+} W\right] U_{s t}
$$

From $\left\{U_{s t}\right\}$, we can introduce the generalized Bell states

$$
\left|\Phi_{s t}\right\rangle=\left(I \otimes U_{s t}^{*}\right)\left|\psi_{+}\right\rangle=\frac{1}{\sqrt{d}} \sum_{i, j}\left(U_{s t}\right)_{i j}^{*}|i j\rangle, \quad\left|\Phi_{00}\right\rangle=\left|\psi_{+}\right\rangle,
$$

where $\left|\Phi_{s t}\right\rangle$ are all maximally entangled states and form a complete orthogonal normalized basis of $\mathscr{t}_{d} \otimes \mathscr{t}_{d}$.

Theorem 4.4. For any quantum state $\rho \in \mathscr{H}_{d} \otimes \mathfrak{H}_{d}$, the fully entangled fraction defined in (4.1) fulfills the following inequality:

$$
\mathcal{F}(\rho) \leq \max _{j}\left\{\lambda_{j}\right\}
$$

where $\lambda_{j}$ s are the eigenvalues of the real part of matrix $M=\left(\begin{array}{cc}T & i T \\ -i T & T\end{array}\right), T$ is a $d^{2} \times d^{2}$ matrix with entries $T_{n, m}=\left\langle\Phi_{n}|\rho| \Phi_{m}\right\rangle$, and $\Phi_{j}$ are the maximally entangled basis states defined in (4.16) [125]. 
Proof. From (4.15), any $d \times d$ unitary matrix $U$ can be represented by $U=\sum_{k=1}^{d^{2}} z_{k} U_{k}$, where $z_{k}=(1 / d) \operatorname{Tr}\left[U_{k}^{\dagger} U\right]$. Define

$$
x_{l}=\left\{\begin{array}{ll}
\operatorname{Re}\left[z_{l}\right], & 1 \leq l \leq d^{2}, \\
\operatorname{Im}\left[z_{l}\right], & d^{2}<l \leq 2 d^{2},
\end{array} \quad U_{l}^{\prime}= \begin{cases}U_{l}, & 1 \leq l \leq d^{2}, \\
i * U_{l}, & d^{2}<l \leq 2 d^{2} .\end{cases}\right.
$$

Then the unitary matrix $U$ can be rewritten as $U=\sum_{k=1}^{2 d^{2}} z_{k} U_{k}^{\prime}$. The necessary condition for the unitary property of $U$ implies that $\sum_{k} x_{k}^{2}=1$. Thus we have

$$
F(\rho) \equiv\left\langle\psi_{+}\left|\left(I \otimes U^{\dagger}\right) \rho(I \otimes U)\right| \psi_{+}\right\rangle=\sum_{m, n=1}^{2 d^{2}} x_{m} x_{n} M_{m n}
$$

where $M_{m n}$ is defined in the theorem. One can deduce that

$$
M_{m n}^{*}=M_{n m}
$$

from the hermiticity of $\rho$.

Taking into account the constraint with an undetermined Lagrange multiplier $\lambda$, we have

$$
\frac{\partial}{\partial x_{k}}\left\{F(\rho)+\lambda\left(\sum_{l} x_{l}^{2}-1\right)\right\}=0
$$

Accounting to (4.20) we have the eigenvalue equation

$$
\sum_{n=1}^{2 d^{2}} \operatorname{Re}\left[M_{k, n}\right] x_{n}=-\lambda x_{k}
$$

Inserting (4.22) into (4.19) results in

$$
\mathcal{F}(\rho)=\max _{U} F \leq \max _{j}\left\{\eta_{j}\right\}
$$

where $\eta_{j}=-\lambda_{j}$ is the corresponding eigenvalues of the real part of the matrix $M$. 


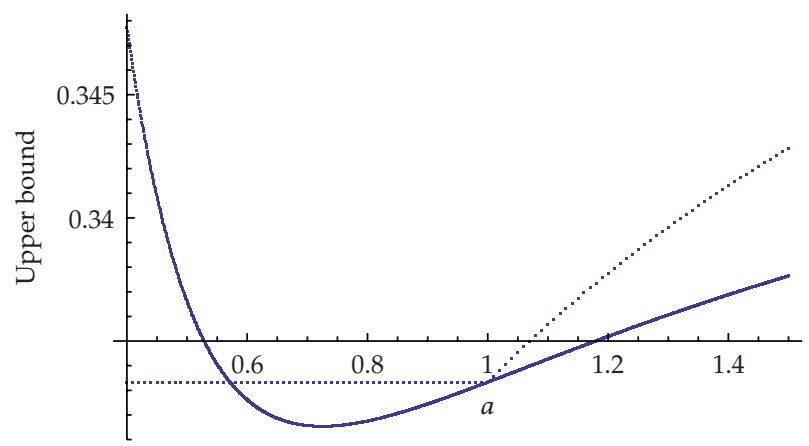

Figure 2: Upper bound of $\mathcal{F}(\rho(a))$ from (4.17) (solid line) and upper bound from (4.4) (dashed line) for state (4.24).

Example 4.5. Horodecki gives a very interesting bound entangled state in [31] as

$$
\rho(a)=\frac{1}{8 a+1}\left(\begin{array}{ccccccccc}
a & 0 & 0 & 0 & a & 0 & 0 & 0 & a \\
0 & a & 0 & 0 & 0 & 0 & 0 & 0 & 0 \\
0 & 0 & a & 0 & 0 & 0 & 0 & 0 & 0 \\
0 & 0 & 0 & a & 0 & 0 & 0 & 0 & 0 \\
a & 0 & 0 & 0 & a & 0 & 0 & 0 & a \\
0 & 0 & 0 & 0 & 0 & a & 0 & 0 & 0 \\
0 & 0 & 0 & 0 & 0 & 0 & \frac{1+a}{2} & 0 & \frac{\sqrt{1-a^{2}}}{2} \\
0 & 0 & 0 & 0 & 0 & 0 & 0 & a & 0 \\
a & 0 & 0 & 0 & a & 0 & \frac{\sqrt{1-a^{2}}}{2} & 0 & \frac{1+a}{2}
\end{array}\right) .
$$

One can easily compare the upper bound obtained in (4.17) and that in (4.4). From Figure 2 we see that, for $0 \leq a<0.572$, the upper bound in (4.17) is larger than that in (4.4). But for $0.572<a<1$ the upper bound in (4.17) is always lower than that in (4.4), which means that the upper bound (4.17) is tighter than (4.4).

In fact, we can drive another upper bound for FEF which will be very tight for weakly mixed-quantum states.

Theorem 4.6. For any bipartite quantum state $\rho \in \mathfrak{H}_{d} \otimes \mathfrak{l}_{d}$, the following inequality holds [125]:

$$
\mathcal{F}(\rho) \leq \frac{1}{d}\left(\operatorname{Tr}\left[\sqrt{\rho_{A}}\right]\right)^{2}
$$

where $\rho_{A}$ is the reduced matrix of $\rho$. 
Proof. Note that in [77] the authors have obtained the FEF for pure state $|\psi\rangle$ as

$$
\mathcal{F}(|\psi\rangle)=\frac{1}{d}\left(\operatorname{Tr}\left[\sqrt{\rho_{A}^{|\psi\rangle}}\right]\right)^{2}
$$

where $\rho_{A}^{|\psi\rangle}$ is the reduced matrix of $|\psi\rangle\langle\psi|$.

For mixed state $\rho=\sum_{i} p_{i} \rho^{i}$, we have

$$
\begin{aligned}
\mathcal{F}(\rho) & =\max _{U}\left\langle\psi_{+}\left|\left(I \otimes U^{\dagger}\right) \rho(I \otimes U)\right| \psi_{+}\right\rangle \leq \sum_{i} p_{i} \max _{U}\left\langle\psi_{+}\left|\left(I \otimes U^{\dagger}\right) \rho^{i}(I \otimes U)\right| \psi_{+}\right\rangle \\
& =\frac{1}{d} \sum_{i} p_{i}\left(\operatorname{Tr}\left[\sqrt{\rho_{A}^{i}}\right]\right)^{2}=\frac{1}{d} \sum_{i}\left(\operatorname{Tr}\left[\sqrt{p_{i} \rho_{A}^{i}}\right]\right)^{2} .
\end{aligned}
$$

Let $\lambda_{i j}$ be the real and nonnegative eigenvalues of the matrix $p_{i} \rho_{A}^{i}$. Recall that for any function $F=\sum_{i}\left(\sum_{j} x_{i j}^{2}\right)^{1 / 2}$ subjected to the constraints $z_{j}=\sum_{i} x_{i j}$ with $x_{i j}$ being real and nonnegative, the inequality $\sum_{j} z_{j}^{2} \leq F^{2}$ holds, from which it follows that

$$
\mathcal{F}(\rho) \leq \frac{1}{d} \sum_{i}\left(\sum_{j} \sqrt{\lambda_{i j}}\right)^{2} \leq \frac{1}{d}\left(\sum_{j} \sqrt{\sum_{i} \lambda_{i j}}\right)^{2}=\frac{1}{d}\left(\operatorname{Tr}\left[\sqrt{\rho_{A}}\right]\right)^{2},
$$

which ends the proof.

\subsection{Fully Entangled Fraction and Concurrence}

The upper bound of FEF has also interesting relations to the entanglement measure concurrence. As shown in [122], the concurrence of a two-qubit quantum state has some kinds of relation with the optimal teleportation fidelity. For quantum state with high dimension, we have the similar relation between them too.

Theorem 4.7. For any bipartite quantum state $\rho \in \mathfrak{H}_{d} \otimes \mathfrak{L}_{d}$, one has [118]

$$
C(\rho) \geq \sqrt{\frac{2 d}{d-1}}\left[\mp(\rho)-\frac{1}{d}\right] .
$$

Proof. In [126], the authors show that, for any pure state $|\psi\rangle \in \mathscr{d}_{A} \otimes \mathfrak{l}_{B}$, the following inequality holds:

$$
C(|\psi\rangle) \geq \sqrt{\frac{2 d}{d-1}}\left(\max _{|\phi\rangle \in \varepsilon}|\langle\psi \mid \phi\rangle|^{2}-\frac{1}{d}\right)
$$

where $\varepsilon$ denotes the set of $d \times d$ dimensional maximally entangled states. 

have

Let $\rho=\sum_{i} p_{i}\left|\phi_{i}\right\rangle\left\langle\phi_{i}\right|$ be the optimal decomposition such that $C(\rho)=\sum_{i} p_{i} C\left(\left|\psi_{i}\right\rangle\right)$. We

$$
\begin{aligned}
C(\rho) & =\sum_{i} p_{i} C\left(\left|\psi_{i}\right\rangle\right) \geq \sum_{i} p_{i} \sqrt{\frac{2 d}{d-1}}\left(\max _{|\phi\rangle \in \varepsilon}\left|\left\langle\psi_{i} \mid \phi\right\rangle\right|^{2}-\frac{1}{\mathrm{~d}}\right) \\
& \geq \sqrt{\frac{2 d}{d-1}}\left(\max _{|\phi\rangle \in \varepsilon} \sum_{i} p_{i}\left|\left\langle\psi_{i} \mid \phi\right\rangle\right|^{2}-\frac{1}{d}\right) \\
& =\sqrt{\frac{2 d}{d-1}}\left(\max _{|\phi\rangle \in \varepsilon}\langle\phi|\rho| \phi\rangle-\frac{1}{d}\right)=\sqrt{\frac{2 d}{d-1}}\left(\mp(\rho)-\frac{1}{d}\right),
\end{aligned}
$$

which ends the proof.

Inequality (4.29) has demonstrated the relation between the lower bound of concurrence and the fully entangled fraction (thus the optimal teleportation fidelity), that is, the fully entangled fraction of a quantum state $\rho$ is limited by its concurrence.

We now consider tripartite case. Let $\rho_{A B C}$ be a state of three-qubit systems denoted by $A, B$, and $C$. We study the upper bound of the FEF, $\mathcal{F}\left(\rho_{A B}\right)$, between qubits $A$ and $B$, and its relations to the concurrence under bipartite partition $A B$ and $C$. For convenience we normalize $\mathcal{F}\left(\rho_{A B}\right)$ to be

$$
\mathcal{F}_{N}\left(\rho_{A B}\right)=\max \left\{2 \mathcal{F}\left(\rho_{A B}\right)-1,0\right\}
$$

Let $C\left(\rho_{A B \mid C}\right)$ denote the concurrence between subsystems $A B$ and $C$.

Theorem 4.8. For any triqubit state $\rho_{A B C}, \mathcal{F}_{N}\left(\rho_{A B}\right)$ satisfies [123]

$$
\mathcal{F}_{N}\left(\rho_{A B}\right) \leq \sqrt{1-C^{2}\left(\rho_{A B \mid C}\right)}
$$

Proof. We first consider the case that $\rho_{\mathrm{ABC}}$ is pure, $\rho_{A B C}=|\psi\rangle_{A B C}\langle\psi|$. By using the Schmidt decomposition between qubits $A, B$, and $C,|\psi\rangle_{A B C}$ can be written as

$$
|\psi\rangle_{A B \mid C}=\sum_{i=1}^{2} \eta_{i}\left|i_{A B}\right\rangle\left|i_{C}\right\rangle, \quad \eta_{1}^{2}+\eta_{2}^{2}=1, \eta_{1} \geq \eta_{2},
$$

for some orthonormalized bases $\left|i_{A B}\right\rangle,\left|i_{C}\right\rangle$ of subsystems $A B, C$, respectively. The reduced density matrix $\rho_{A B}$ has the form

$$
\rho_{A B}=\operatorname{Tr}_{C}\left[\rho_{A B C}\right]=\sum_{i=1}^{2} \eta_{i}^{2}\left|i_{A B}\right\rangle\left\langle i_{A B}\right|=U^{T} \Lambda U^{*}
$$

where $\Lambda$ is a $4 \times 4$ diagonal matrix with diagonal elements $\left\{\eta_{1}^{2}, \eta_{2}^{2}, 0,0\right\}, U$ is a unitary matrix, and $U^{*}$ denotes the conjugation of $U$. 
The FEF of the two-qubit state $\rho_{A B}$ can be calculated by using formula (4.10) or the one in [122]. Let

$$
M=\frac{1}{\sqrt{2}}\left(\begin{array}{cccc}
1 & 0 & 0 & i \\
0 & i & -1 & 0 \\
0 & i & 1 & 0 \\
1 & 0 & 0 & -i
\end{array}\right)
$$

be the $4 \times 4$ matrix constituted by the four Bell bases. The FEF of $\rho_{A B}$ can be written as

$$
\begin{aligned}
\mathcal{F}\left(\rho_{A B}\right) & =\eta_{\max }\left(\operatorname{Re}\left\{M^{\dagger} \rho_{A B} M\right\}\right)=\frac{1}{2} \eta_{\max }\left(M^{\dagger} \rho_{A B} M+M^{T} \rho_{A B}^{*} M^{*}\right) \\
& \leq \frac{1}{2}\left[\eta_{\max }\left(M^{\dagger} U^{T} \Lambda U^{*} M\right)+\eta_{\max }\left(M^{T} U^{\dagger} \Lambda U M^{*}\right)\right]=\eta_{1}^{2},
\end{aligned}
$$

where $\eta_{\max }(X)$ stands for the maximal eigenvalues of the matrix $X$.

For pure state (4.34) in bipartite partition $A B$ and $C$, we have

$$
C\left(|\psi\rangle_{A B \mid C}\right)=\sqrt{2\left(1-\operatorname{Tr}\left[\rho_{A B}^{2}\right]\right)}=2 \eta_{1} \eta_{2}
$$

From (4.32), (4.37), and (4.38) we get

$$
\mathcal{F}_{N}\left(\rho_{A B}\right) \leq \sqrt{1-C^{2}\left(|\psi\rangle_{A B \mid C}\right)}
$$

We now prove that the above inequality (4.39) also holds for mixed state $\rho_{A B C}$. Let $\rho_{A B C}=\sum_{i} p_{i}\left|\psi_{i}\right\rangle_{A B C}\left\langle\psi_{i}\right|$ be the optimal decomposition of $\rho_{A B C}$ such that $C\left(\rho_{A B \mid C}\right)=$ $\sum_{i} p_{i} C\left(\left|\psi_{i}\right\rangle\right)_{A B \mid C}$. We have

$$
\begin{aligned}
\mathcal{F}_{N}\left(\rho_{A B}\right) & \leq \sum_{i} p_{i} \mathcal{F}_{N}\left(\rho_{A B}^{i}\right) \leq \sum_{i} p_{i} \sqrt{1-C^{2}\left(\rho_{A B \mid C}^{i}\right)} \\
& \leq \sqrt{1-\sum_{i} p_{i} C^{2}\left(\rho_{A B \mid C}^{i}\right)} \leq \sqrt{1-C^{2}\left(\rho_{A B \mid C}\right)},
\end{aligned}
$$

where $\rho_{A B \mid C}^{i}=\left|\psi_{i}\right\rangle_{A B C}\left\langle\psi_{i}\right|$ and $\rho_{A B}^{i}=\operatorname{Tr} r_{C}\left[\rho_{A B \mid C}^{i}\right]$.

From Theorem 4.8 we see that the FEF of qubits $A$ and $B$ are bounded by the concurrence between qubits $A, B$, and qubit $C$. The upper bound of FEF for $\rho_{A B}$ decreases when the entanglement between qubits $A, B$, and $C$ increases. As an example, we consider 


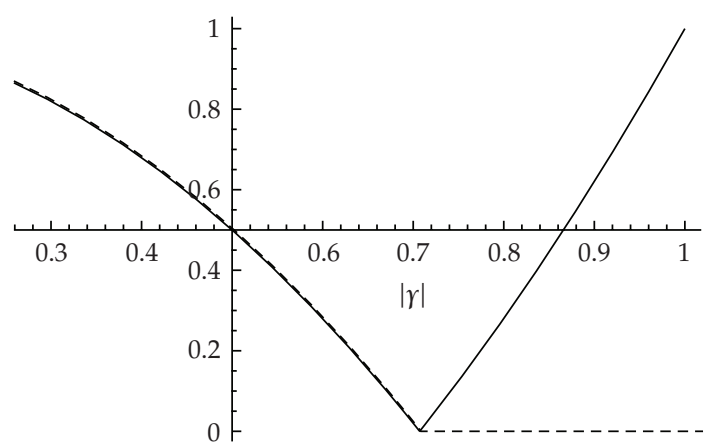

Figure 3: $\mathcal{F}_{N}\left(\rho_{A B}^{W^{\prime}}\right)$ (dashed line) and upper bound $\sqrt{1-C^{2}\left(\left|W^{\prime}\right\rangle_{A B \mid C}\right)}$ (solid line) of state $\left|W^{\prime}\right\rangle_{A B \mid C}$ at $|\alpha|=$ $|\beta|$.

the generalized $W$ state defined by $\left|W^{\prime}\right\rangle=\alpha|100\rangle+\beta|010\rangle+\gamma|001\rangle,|\alpha|^{2}+|\beta|^{2}+|\gamma|^{2}=1$. The reduced density matrix is given by

$$
\rho_{A B}^{W^{\prime}}=\left(\begin{array}{cccc}
|\gamma|^{2} & 0 & 0 & 0 \\
0 & |\beta|^{2} & \alpha^{*} \beta & 0 \\
0 & \alpha \beta^{*} & |\alpha|^{2} & 0 \\
0 & 0 & 0 & 0
\end{array}\right) .
$$

The FEF of $\rho_{A B}^{W^{\prime}}$ is given by

$$
\mathcal{F}_{N}\left(\rho_{A B}^{W^{\prime}}\right)=-\frac{1}{2}+2|\alpha||\beta|+\left.\frac{1}{2}|| \alpha\right|^{2}+|\beta|^{2}-|\gamma|^{2} \mid
$$

while the concurrence of $\left|W^{\prime}\right\rangle$ has the form $C_{A B \mid C}\left(\left|W^{\prime}\right\rangle\right)=2|\gamma| \sqrt{|\alpha|^{2}+|\beta|^{2}}$. We see that (4.33) always holds. In particular for $|\alpha|=|\beta|$ and $|\gamma| \leq \sqrt{2} / 2$, the inequality (4.33) is saturated (see Figure 3).

\subsection{Improvement of Entanglement Distillation Protocol}

The upper bound can give rise to not only an estimation of the fidelity in quantum information processing such as teleportation, but also an interesting application in entanglement distillation of quantum states. In [77] a generalized distillation protocol has been presented. It is shown that a quantum state $\rho$ violating the reduction criterion can always be distilled. For such states if their single fraction of entanglement $F(\rho)=\left\langle\psi_{+}|\rho| \psi_{+}\right\rangle$is greater than $1 / d$, then one can distill these states directly by using the generalized distillation protocol. If the FEF (the largest value of single fraction of entanglement under local unitary transformations) is less than or equal to $1 / d$, then a proper filtering operation has to be used at first to transform $\rho$ to another state $\rho^{\prime}$ so that $F\left(\rho^{\prime}\right)>1 / d$. For $d=2$, one can compute FEF analytically according to the corollary. For $d \geq 3$ our upper bound (4.4) can supply a necessary condition in the distillation. 


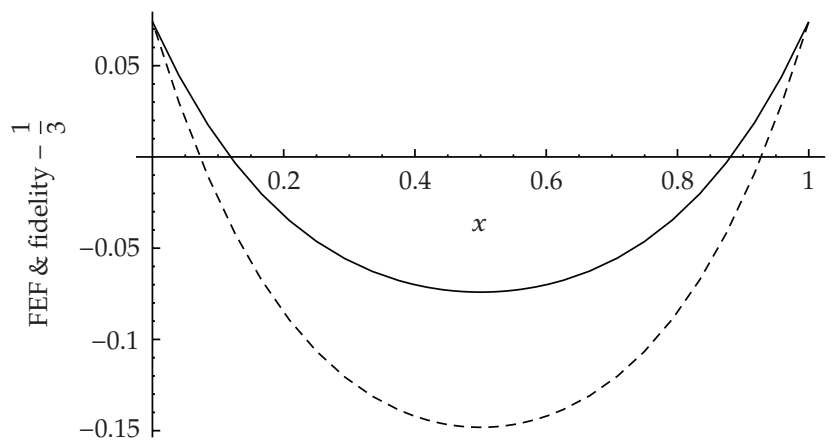

Figure 4: Upper bound of $\mathcal{F}(\rho)-1 / 3$ from (4.4) (solid line) and fidelity $F(\rho)-1 / 3$ (dashed line) for state (4.43).

Theorem 4.9. For an entangled state $\rho \in \mathscr{d} \otimes \mathscr{d}$ violating the reduction criterion, if the upper bound (4.4) is less than or equal to $1 / d$, then the filtering operation has to be applied before using the generalized distillation protocol [123].

As an example, we consider a $3 \times 3$ state

$$
\rho=\frac{8}{9} \sigma+\frac{1}{9}\left|\psi_{+}\right\rangle\left\langle\psi_{+}\right|
$$

where $\sigma=(x|0\rangle\langle 0|+(1-x)| 1\rangle\langle 1|) \otimes(x|0\rangle\langle 0|+(1-x)| 1\rangle\langle 1|)$. It is direct to verify that $\rho$ violates the reduction criterion for $0 \leq x \leq 1$, as $\left(\rho_{1} \otimes I\right)-\rho$ has a negative eigenvalue $-2 / 27$. Therefore the state is distillable. From Figure 4, we see that for $0 \leq x<0.0722$ and $0.9278<x \leq 1$, the fidelity is already greater than $1 / 3$; thus the generalized distillation protocol can be applied without the filtering operation. However for $0.1188 \leq x \leq 0.8811$, even the upper bound of the fully entangled fraction is less than or equal to $1 / 3$; hence the filtering operation has to be applied first, before using the generalized distillation protocol.

Moreover, the lower bounds of concurrence can be also used to study the distillability of quantum states. Based on the positive partial transpose (PPT) criterion, a necessary and sufficient condition for the distillability was proposed in [127], which is not operational in general. An alternative distillability criterion based on the bound $\tau_{2}$ in (3.19) can be obtained to improve the operationality.

Theorem 4.10. A bipartite quantum state $\rho$ is distillable if and only if $\tau_{2}\left(\rho^{\otimes N}\right)>0$ for some number $N$ [67].

Proof. It was shown in [127] that a density matrix $\rho$ is distillable if and only if there are some projectors $P, Q$ that map high-dimensional spaces to two-dimensional ones and some number $N$ such that the state $P \otimes Q \rho^{\otimes N} P \otimes Q$ is entangled [127]. Thus if $\tau_{2}\left(\rho^{\otimes N}\right)>0$, then there exists one submatrix of matrix $\rho^{\otimes N}$, similar to (3.20), which has nonzero $\tau_{2}$ and is entangled in a $2 \otimes 2$ space; hence $\rho$ is distillable. 
Corollary 4.11. (a) The lower bound $\tau_{2}(\rho)>0$ is a sufficient condition for the distillability of any bipartite state $\rho$.

(b) The lower bound $\tau_{2}(\rho)=0$ is a necessary condition for separability of any bipartite state $\rho$.

Remark 4.12. Corollary 4.11 directly follows from Theorem 4.10 and this case is referred to as one distillable [128]. The problem of whether non-PPT (NPPT) nondistillable states exist is studied numerically in $[128,129]$. By using Theorem 4.10 , although it seems impossible to solve the problem completely, it is easy to judge the distillability of a state under condition that is one distillable.

The lower bound $\tau_{2}$, PPT criterion, separability, and distillability for any bipartite quantum state $\rho$ have the following relations: if $\tau_{2}(\rho)>0$, then $\rho$ is entangled. If $\rho$ is separable, then it is PPT. If $\tau_{2}(\rho)>0$, then $\rho$ is distillable. If $\rho$ is distillable, then it is NPPT. From the last two propositions it follows that if $\rho$ is PPT, then $\tau_{2}(\rho)=0$, that is, if $\tau_{2}(\rho)>0$, then $\rho$ is NPPT.

Theorem 4.13. For any pure tripartite state $|\phi\rangle_{A B C}$ in arbitrary $d \otimes d \otimes d$ dimensional spaces, bound $\tau_{2}$ satisfies [67]

$$
\tau_{2}\left(\rho_{A B}\right)+\tau_{2}\left(\rho_{A C}\right) \leq \tau_{2}\left(\rho_{A: B C}\right)
$$

where $\rho_{A B}=\operatorname{Tr}_{C}\left(|\phi\rangle_{A B C}\langle\phi|\right), \rho_{A C}=\operatorname{Tr}_{B}\left(|\phi\rangle_{A B C}\langle\phi|\right)$, and $\rho_{A: B C}=\operatorname{Tr}_{B C}\left(|\phi\rangle_{A B C}\langle\phi|\right)$.

Proof. Since $C_{m n}^{2} \leq\left(\lambda_{m n}^{(1)}\right)^{2} \leq \sum_{i=1}^{4}\left(\lambda_{m n}^{(i)}\right)^{2}=\operatorname{Tr}\left(\rho \tilde{\rho}_{m n}\right)$, one can derive the inequality

$$
\tau\left(\rho_{A B}\right)+\tau\left(\rho_{A C}\right) \leq \sum_{l, k}^{D} \operatorname{Tr}\left[\rho_{A B}\left(\tilde{\rho}_{A B}\right)_{l k}\right]+\sum_{p, q}^{D} \operatorname{Tr}\left[\rho_{A C}\left(\tilde{\rho}_{A C}\right)_{p q}\right]
$$

where $D=d(d-1) / 2$. Note that $\sum_{l k} \operatorname{Tr}\left[\rho_{A B}\left(\tilde{\rho}_{A B}\right)_{l k}\right] \leq 1-\operatorname{Tr} \rho_{A}^{2}-\operatorname{Tr} \rho_{B}^{2}+\operatorname{Tr} \rho_{C}^{2}$ and $\sum_{p q} \operatorname{Tr}\left[\rho_{A C}\left(\tilde{\rho}_{A C}\right)_{p q}\right] \leq 1-\operatorname{Tr} \rho_{A}^{2}+\operatorname{Tr} \rho_{B}^{2}-\operatorname{Tr} \rho_{C^{\prime}}^{2}$, where $l, p k, q,=1, \ldots, D$. By using the similar analysis in [113], one has that the right-hand side of (4.45) is equal to $2\left(1-\operatorname{Tr} \rho_{A}^{2}\right)=C^{2}\left(\rho_{A: B C}\right)$. Taking into account that $\tau_{2}\left(\rho_{A: B C}\right)=\mathcal{C}^{2}\left(\rho_{A: B C}\right)$ for a pure state, one obtains inequality (4.44).

Generally for any pure multipartite quantum state $\rho_{A B_{1} B_{2} \cdots b_{n}}$, one has the following monogamy inequality:

$$
\tau_{2}\left(\rho_{A B_{1}}\right)+\tau_{2}\left(\rho_{A B_{2}}\right)+\cdots+\tau_{2}\left(\rho_{A B_{n}}\right) \leq \tau_{2}\left(\rho_{A: B_{1} B_{2} \cdots B_{n}}\right) .
$$

\section{Summary and Conclusion}

We have introduced some recent results on three aspects in quantum information theory. The first one is the separability of quantum states. New criteria to detect more entanglements have been discussed. The normal forms of quantum states have been also studied, which helps in investigating the separability of quantum states. Moreover, since many kinds of quantum states can be transformed into the same normal forms, quantum states can be classified in terms of the normal forms. For the well-known entanglement measure concurrence, we have discussed the tight lower and upper bounds. It turns out that, although one cannot distill 
a singlet from many pairs of bound entangled states, the concurrence and tangle of two entangled quantum states are always larger than those of one, even if both of two entangled quantum states are bound entangled. Related to the optimal teleportation fidelity, upper bounds for the fully entangled fraction have been studied, which can be used to improve the distillation protocol. Interesting relations between fully entangled fraction and concurrence have been also introduced. All these related problems in the theory of quantum entanglement have not been completely solved yet. Many problems remain open concerning the physical properties and mathematical structures of quantum entanglement, and the applications of entangled states in information processing.

\section{References}

[1] A. Einstein, B. Podolsky, and N. Rosen, "Can quantum-mechanical description of physical reality be considered complete?" Physical Review, vol. 47, no. 10, pp. 777-780, 1935.

[2] A. Peres, Quantum Mechanics: Concepts and Methods, Kluwer Academic Publishers, Dordrecht, The Netherlands, 1993.

[3] M. A. Nielsen and I. L. Chuang, Quantum Computation and Quantum Information, Cambridge University Press, Cambridge, UK, 2000.

[4] D. P. Di Vincenzo, “Quantum computation," Science, vol. 270, pp. 255-261, 1995.

[5] C. H. Bennett, G. Brassard, C. Crépeau, R. Jozsa, A. Peres, and W. K. Wootters, "Teleporting an unknown quantum state via dual classical and Einstein-Podolsky-Rosen channels," Physical Review Letters, vol. 70, no. 13, pp. 1895-1899, 1993.

[6] S. Albeberio and S. M. Fei, "Teleportation of general finite-dimensional quantum systems," Physics Letters A, vol. 276, pp. 8-11, 2000.

[7] G. M. D'Ariano, P. Lo Presti, and M. F. Sacchi, "Bell measurements and observables," Physics Letters A, vol. 272, no. 1-2, pp. 32-38, 2000.

[8] M. Horodecki, P. Horodecki, and R. Horodecki, "General teleportation channel, singlet fraction, and quasidistillation," Physical Review A, vol. 60, no. 3, pp. 1888-1898, 1999.

[9] S. Albeverio, S.-M. Fei, and W.-L. Yang, "Optimal teleportation based on bell measurements," Physical Review A, vol. 66, no. 1, Article ID 012301, 4 pages, 2002.

[10] C. H. Bennett and S. J. Wiesner, "Communication via one- and two-particle operators on EinsteinPodolsky-Rosen states," Physical Review Letters, vol. 69, no. 20, pp. 2881-2884, 1992.

[11] A. K. Ekert, "Quantum cryptography based on Bell's theorem," Physical Review Letters, vol. 67, no. 6, pp. 661-663, 1991.

[12] D. Deutsch, A. Ekert, R. Jozsa, C. Macchiavello, S. Popescu, and A. Sanpera, "Quantum privacy amplification and the security of quantum cryptography over noisy channels," Physical Review Letters, vol. 77, no. 13, pp. 2818-2821, 1996.

[13] C. A. Fuchs, N. Gisin, R. B. Griffiths, C.-S. Niu, and A. Peres, “Optimal eavesdropping in quantum cryptography. I. Information bound and optimal strategy," Physical Review A, vol. 56, no. 2, pp. 1163 1172, 1997.

[14] M. Zukowski, A. Zeilinger, M. A. Horne, and A. K. Ekert, “"'Event-ready-detectors” Bell experiment via entanglement swapping," Physical Review Letters, vol. 71, no. 26, pp. 4287-4290, 1993.

[15] S. Bose, V. Vedral, and P. L. Knight, "Multiparticle generalization of entanglement swapping," Physical Review A, vol. 57, no. 2, pp. 822-829, 1998.

[16] S. Bose, V. Vedral, and P. L. Knight, "Purification via entanglement swapping and conserved entanglement," Physical Review A, vol. 60, no. 1, pp. 194-197, 1999.

[17] B.-S. Shi, Y.-K. Jiang, and G.-C. Guo, "Optimal entanglement purification via entanglement swapping," Physical Review A, vol. 62, no. 5, Article ID 054301, 2000.

[18] L. Hardy and D. D. Song, "Entanglement-swapping chains for general pure states," Physical Review A, vol. 62, no. 5, Article ID 052315, 2000.

[19] C. H. Bennett, D. P. DiVincenzo, P. W. Shor, J. A. Smolin, B. M. Terhal, and W. K. Wootters, “Remote state preparation," Physical Review Letters, vol. 87, no. 7, Article ID 077902, 4 pages, 2001.

[20] B.-S. Shi and A. Tomita, "Remote state preparation of an entangled state," Journal of Optics B, vol. 4, no. 6, pp. 380-382, 2002. 
[21] J. M Liu and Y. Z. Wang, "Probabilistic remote state preparation by W states," Chinese Physics, vol. 13, pp. 147-152, 2004.

[22] D. W. Leung and P. W. Shor, "Oblivious remote state preparation," Physical Review Letters, vol. 90, no. 12, Article ID 127905, 4 pages, 2003.

[23] D. W. Berry and B. C. Sanders, "Optimal remote state preparation," Physical Review Letters, vol. 90, no. 5, Article ID 057901, 4 pages, 2003.

[24] M.-Y. Ye, Y.-S. Zhang, and G.-C. Guo, "Faithful remote state preparation using finite classical bits and a nonmaximally entangled state," Physical Review A, vol. 69, no. 2, Article ID 022310, 4 pages, 2004.

[25] R. Horodecki, P. Horodecki, M. Horodecki, and K. Horodecki, "Quantum entanglement," Reviews of Modern Physics, vol. 81, no. 2, pp. 865-942, 2009.

[26] O. Gühne and G. Tóth, "Entanglement detection," Physics Reports, vol. 474, no. 1-6, pp. 1-75, 2009.

[27] S. Albeverio and S.-M. Fei, "A note on invariants and entanglements," Journal of Optics B, vol. 3, no. 4, pp. 223-227, 2001.

[28] R. F. Werner, "Quantum states with Einstein-Podolsky-Rosen correlations admitting a hiddenvariable model," Physical Review A, vol. 40, no. 8, pp. 4277-4281, 1989.

[29] A. Peres, "Separability criterion for density matrices," Physical Review Letters, vol. 77, no. 8, pp. 14131415, 1996.

[30] M. Horodecki, P. Horodecki, and R. Horodecki, "Separability of mixed states: necessary and sufficient conditions," Physics Letters A, vol. 223, no. 1-2, pp. 1-8, 1996.

[31] P. Horodecki, "Separability criterion and inseparable mixed states with positive partial transposition," Physics Letters A, vol. 232, no. 5, pp. 333-339, 1997.

[32] O. Rudolph, "Some properties of the computable cross-norm criterion for separability," Physical Review A, vol. 67, no. 3, Article ID 032312, 2003.

[33] K. Chen and L.-A. Wu, "A matrix realignment method for recognizing entanglement," Quantum Information and Computation, vol. 3, no. 3, pp. 193-202, 2003.

[34] M. Horodecki, P. Horodecki, and R. Horodecki, "Separability of mixed quantum states: linear contractions and permutation criteria," Open Systems $\mathcal{E}$ Information Dynamics, vol. 13, no. 1, pp. 103$111,2006$.

[35] K. Chen and L.-A. Wu, "The generalized partial transposition criterion for separability of multipartite quantum states," Physics Letters A, vol. 306, no. 1, pp. 14-20, 2002.

[36] O. Rudolph, "Some properties of the computable cross-norm criterion for separability," Physical Review A, vol. 67, no. 3, Article ID 032312, 2003.

[37] K. Chen and L.-A. Wu, "Test for entanglement using physically observable witness operators and positive maps," Physical Review A, vol. 69, no. 2, Article ID 022312, 2004.

[38] L. Clarisse and P. Wocjan, "On independent permutation separability criteria," Quantum Information $\mathcal{E}$ Computation, vol. 6, no. 3, pp. 277-288, 2006.

[39] S. Albeverio, K. Chen, and S.-M. Fei, "Generalized reduction criterion for separability of quantum states," Physical Review A, vol. 68, no. 6, Article ID 062313, 2003.

[40] M. Horodecki and P. Horodecki, "Reduction criterion of separability and limits for a class of distillation protocols," Physical Review A, vol. 59, no. 6, pp. 4206-4216, 1999.

[41] N. J. Cerf, C. Adami, and R. M. Gingrich, "Reduction criterion for separability," Physical Review A, vol. 60, no. 2, pp. 898-909, 1999.

[42] M. A. Nielsen and J. Kempe, "Separable states are more disordered globally than locally," Physical Review Letters, vol. 86, no. 22, pp. 5184-5187, 2001.

[43] H. F. Hofmann and S. Takeuchi, "Violation of local uncertainty relations as a signature of entanglement," Physical Review A, vol. 68, no. 3, Article ID 032103, 2003.

[44] O. Gühne, M. Mechler, G. Tóth, and P. Adam, "Entanglement criteria based on local uncertainty relations are strictly stronger than the computable cross norm criterion," Physical Review A, vol. 74, no. 1, Article ID 010301, 2006.

[45] O. Gühne, "Characterizing entanglement via uncertainty relations," Physical Review Letters, vol. 92, no. 11, Article ID 117903, 2004.

[46] C.-J. Zhang, Y.-S. Zhang, S. Zhang, and G.-C. Guo, “Optimal entanglement witnesses based on local orthogonal observables," Physical Review A, vol. 76, no. 1, Article ID 012334, 2007.

[47] J. I. De Vicente, "Separability criteria based on the Bloch representation of density matrices," Quantum Information E Computation, vol. 7, no. 7, pp. 624-638, 2007.

[48] J. I. De Vicente, "Further results on entanglement detection and quantification from the correlation matrix criterion," Journal of Physics A, vol. 41, no. 6, Article ID 065309, 2008. 
[49] O. Gühne, P. Hyllus, O. Gittsovich, and J. Eisert, "Covariance matrices and the separability problem," Physical Review Letters, vol. 99, no. 13, Article ID 130504, 2007.

[50] O. Gittsovich, O. Gühne, P. Hyllus, and J. Eisert, "Unifying several separability conditions using the covariance matrix criterion," Physical Review A, vol. 78, no. 5, Article ID 052319, 2008.

[51] C. H. Bennett, G. Brassard, S. Popescu, B. Schumacher, J. Smolin, and W. K. Wootters, "Purification of noisy entanglement and faithful teleportation via noisy channels," Physical Review Letters, vol. 78, p. 2031, 1996.

[52] D. Bruß, “Characterizing entanglement," Journal of Mathematical Physics, vol. 43, no. 9, pp. 4237-4251, 2002.

[53] W. K. Wootters, "Entanglement of formation of an arbitrary state of two qubits," Physical Review Letters, vol. 80, no. 10, pp. 2245-2248, 1998.

[54] B. M. Terhal and K. G. H. Vollbrecht, "Entanglement of formation for isotropic states," Physical Review Letters, vol. 85, no. 12, pp. 2625-2628, 2000.

[55] S.-M. Fei, J. Jost, X. Li-Jost, and G.-F. Wang, "Entanglement of formation for a class of quantum states," Physics Letters A, vol. 310, no. 5-6, pp. 333-338, 2003.

[56] S.-M. Fei and X. Li-Jost, "A class of special matrices and quantum entanglement," Reports on Mathematical Physics, vol. 53, no. 2, pp. 195-210, 2004.

[57] S.-M. Fei, Z.-X. Wang, and H. Zhao, "A note on entanglement of formation and generalized concurrence," Physics Letters A, vol. 329, no. 6, pp. 414-419, 2004.

[58] P. Rungta and C. M. Caves, "Concurrence-based entanglement measures for isotropic states," Physical Review A, vol. 67, no. 1, Article ID 012307, 9 pages, 2003.

[59] F. Mintert, M. Kus, and A. Buchleitner, "Concurrence of mixed bipartite quantum states in arbitrary dimensions," Physical Review Letters, vol. 92, no. 16, Article ID 167902, 2004.

[60] K. Chen, S. Albeverio, and S.-M. Fei, "Entanglement of formation of bipartite quantum states," Physical Review Letters, vol. 95, no. 21, Article ID 210501, 2005.

[61] K. Chen, S. Albeverio, and S.-M. Fei, "Concurrence of arbitrary dimensional bipartite quantum states," Physical Review Letters, vol. 95, no. 4, Article ID 040504, 2005.

[62] H.-P. Breuer, "Separability criteria and bounds for entanglement measures," Journal of Physics A, vol. 39, no. 38, pp. 11847-11860, 2006.

[63] H.-P. Breuer, "Optimal entanglement criterion for mixed quantum states," Physical Review Letters, vol. 97, no. 8, Article ID 080501, 2006.

[64] J. I. De Vicente, “Lower bounds on concurrence and separability conditions," Physical Review A, vol. 75, no. 5, Article ID 052320, 2007.

[65] X.-H. Gao, S.-M. Fei, and K. Wu, "Lower bounds of concurrence for tripartite quantum systems," Physical Review A, vol. 74, no. 5, Article ID 050303, 2006.

[66] E. Gerjuoy, "Lower bound on entanglement of formation for the qubit-qudit system," Physical Review A, vol. 67, no. 5, Article ID 052308, 10 pages, 2003.

[67] Y.-C. Ou, H. Fan, and S.-M. Fei, "Proper monogamy inequality for arbitrary pure quantum states," Physical Review A, vol. 78, no. 1, Article ID 012311, 2008.

[68] J. I. De Vicente, "Further results on entanglement detection and quantification from the correlation matrix criterion," Journal of Physics A, vol. 41, no. 6, Article ID 065309, 2008.

[69] F. Mintert, M. Kus, and A. Buchleitner, "Concurrence of mixed multipartite quantum states," Physical Review Letters, vol. 95, no. 26, Article ID 260502, 2005.

[70] S. P. Walborn, P. H. Souto Ribeiro, L. Davidovich, F. Mintert, and A. Buchleitner, "Experimental determination of entanglement with a single measurement," Nature, vol. 440, no. 7087, pp. 10221024, 2006.

[71] S. P. Walborn, P. H. S. Ribeiro, L. Davidovich, F. Mintert, and A. Buchleitner, "Experimental determination of entanglement by a projective measurement," Physical Review A, vol. 75, no. 3, Article ID 032338, 2007.

[72] S.-M. Fei, M.-J. Zhao, K. Chen, and Z.-X. Wang, “Experimental determination of entanglement for arbitrary pure states," Physical Review A, vol. 80, no. 3, Article ID 032320, 2009.

[73] C. H. Bennett, G. Brassard, S. Popescu, B. Schumacher, J. A. Smolin, and W. K. Wootters, "Purification of noisy entanglement and faithful teleportation via noisy channels," Physical Review Letters, vol. 76, no. 5, pp. 722-725, 1996.

[74] M. Horodecki, P. Horodecki, and R. Horodecki, "Inseparable two spin-1/2 density matrices can be distilled to a singlet form," Physical Review Letters, vol. 78, no. 4, pp. 574-577, 1997. 
[75] D. Deutsch, A. Ekert, R. Jozsa, C. Macchiavello, S. Popescu, and A. Sanpera, "Quantum privacy amplification and the security of quantum cryptography over noisy channels," Physical Review Letters, vol. 77, no. 13, pp. 2818-2821, 1996.

[76] C. H. Bennett, D. P. DiVincenzo, J. A. Smolin, and W. K. Wootters, "Mixed-state entanglement and quantum error correction," Physical Review A, vol. 54, no. 5, pp. 3824-3851, 1996.

[77] M. Horodecki and P. Horodecki, "Reduction criterion of separability and limits for a class of distillation protocols," Physical Review A, vol. 59, no. 6, pp. 4206-4216, 1999.

[78] J. Priskill, Quantum Information and Quantum Computation, California Institute of Technology, 1998.

[79] A. Acin, A. Andrianov, L. Costa, E. Jane, J. I. Latorre, and R. Tarrach, "Generalized Schmidt decomposition and classification of three-quantum-bit states," Physical Review Letters, vol. 85, no. 7, pp. 1560-1563, 2000.

[80] A. Sanpera, R. Tarrach, and G. Vidal, "Local description of quantum inseparability," Physical Review A, vol. 58 , no. 2, pp. 826-830, 1998.

[81] N. J. Cerf, C. Adami, and R. M. Gingrich, "Reduction criterion for separability," Physical Review A, vol. 60, no. 2, pp. 898-909, 1999.

[82] M. Horodecki and P. Horodecki, Physical Review A, vol. 54, p. 2406, 1999.

[83] O. Rudolph, "On the cross norm criterion for separability," Journal of Physics A, vol. 36, no. 21, p. $5825,2003$.

[84] F. T. Hioe and J. H. Eberly, "N-level coherence vector and higher conservation laws in quantum optics and quantum mechanics," Physical Review Letters, vol. 47, no. 12, pp. 838-841, 1981.

[85] W. Greiner and B. Muller, Quantum Mechanics: Symmetries, Springer, Berlin, Germany, 1989.

[86] J. E. Harriman, “Geometry of density matrices. I. Definitions, N matrices and 1 matrices," Physical Review A, vol. 17, no. 4, pp. 1249-1256, 1978.

[87] M. Li, S.-M. Fei, and Z.-X. Wang, "Separability of tripartite quantum system," International Journal of Quantum Information, vol. 6, no. 4, pp. 859-866, 2008.

[88] S. Wu and J. Anandan, "Some aspects ot separability," Physics Letters A, vol. 297, no. 1-2, pp. 4-8, 2002.

[89] O. Rudolph, "Further results on the cross norm criterion for separability," Quantum Information Processing, vol. 4, no. 3, pp. 219-239, 2005.

[90] M. Li, S.-M. Fei, and Z.-X. Wang, "Separability and entanglement of quantum states based on covariance matrices," Journal of Physics A, vol. 41, no. 20, Article ID 202002, 2008.

[91] R. A. Horn and C. R. Johnson, Matrix Analysis, Cambridge University Press, Cambridge, UK, 1985.

[92] S. Yu and N. Liu, "Entanglement detection by local orthogonal observables," Physical Review Letters, vol. 95, no. 15, Article ID 150504, 2005.

[93] F. Verstraete, J. Dehaene, and B. De Moor, "Normal forms and entanglement measures for multipartite quantum states," Physical Review A, vol. 68, no. 1, Article ID 012103, 2003.

[94] J. M. Leinaas, J. Myrheim, and E. Ovrum, “Geometrical aspects of entanglement," Physical Review A, vol. 74, no. 1, Article ID 012313, 2006.

[95] F. Verstraete, Ph. K. thesis, Katholieke Universiteit Leuven, 2002.

[96] J. I. De Vicente, "Separability criteria based on the bloch representation of density matrices," Quantum Information and Computation, vol. 7, no. 7, pp. 624-638, 2007.

[97] A. S. M. Hassan and P. S. Joag, "Separability criterion for multipartite quantum states based on the bloch representation of density matrices," Quantum Information and Computation, vol. 8, no. 8-9, pp. 773-790, 2008.

[98] M. Li, S.-M. Fei, and Z.-X. Wang, "A note on normal forms of quantum states and separability," Communications in Theoretical Physics, vol. 50, no. 6, pp. 1307-1311, 2008.

[99] G. Vidal, D. Jonathan, and M. A. Nielsen, "Approximate transformations and robust manipulation of bipartite pure-state entanglement," Physical Review A, vol. 62, no. 1, Article ID 012304, 10 pages, 2000.

[100] V. Vedral and M. B. Plenio, "Entanglement measures and purification procedures," Physical Review A, vol. 57, no. 3, pp. 1619-1633, 1998.

[101] S. Hill and W. K. Wootters, "Entanglement of a pair of quantum bits," Physical Review Letters, vol. 78, no. 26, pp. 5022-5025, 1997.

[102] A. Osterloh, L. Amico, G. Falci, and R. Fazio, "Scaling of entanglement close to a quantum phase transition," Nature, vol. 416, no. 6881, pp. 608-610, 2002.

[103] L.-A. Wu, M. S. Sarandy, and D. A. Lidar, "Quantum phase transitions and bipartite entanglement," Physical Review Letters, vol. 93, no. 25, Article ID 250404, 2004. 
[104] S. Ghosh, T. F. Rosenbaum, G. Aeppli, and S. N. Coppersmith, "Entangled quantum state of magnetic dipoles," Nature, vol. 425, no. 6953, pp. 48-51, 2003.

[105] V. Vedral, "Entanglement hits the big time," Nature, vol. 425, no. 6953, pp. 28-29, 2003.

[106] F. Mintert, M. Kus, and A. Buchleitner, "Concurrence of mixed bipartite quantum states in arbitrary dimensions," Physical Review Letters, vol. 92, no. 16, Article ID 167902, 2004.

[107] F. Mintert, Ph.D. thesis, Munich University, Munich, Germany, 2004.

[108] P. Rungta, V. Bužek, C. M. Caves, M. Hillery, and G. J. Milburn, “Universal state inversion and concurrence in arbitrary dimensions," Physical Review A, vol. 64, no. 4, Article ID 042315, 2001.

[109] A. Uhlmann, "Fidelity and concurrence of conjugated states," Physical Review A, vol. 62, no. 3, Article ID $032307,2000$.

[110] L. Aolita and F. Mintert, "Measuring multipartite concurrence with a single factorizable observable," Physical Review Letters, vol. 97, no. 5, Article ID 050501, 2006.

[111] A. R. R. Carvalho, F. Mintert, and A. Buchleitner, "Decoherence and multipartite entanglement," Physical Review Letters, vol. 93, no. 23, Article ID 230501, 2004.

[112] M. Li, S.-M. Fei, and Z.-X. Wang, "A lower bound of concurrence for multipartite quantum states," Journal of Physics A, vol. 42, no. 14, Article ID 145303, 2009.

[113] V. Coffman, J. Kundu, and W. K. Wootters, "Distributed entanglement," Physical Review A, vol. 61, no. 5, Article ID 052306, 2000.

[114] M. Zukowski, A. Zeilinger, M. A. Horne, and A. K. Ekert, ““"Event-ready-detectors” Bellexperiment via entanglement swapping," Physical Review Letters, vol. 71, no. 26, pp. 4287-4290, 1993.

[115] J.-M. Cai, Z.-W. Zhou, S. Zhang, and G.-C. Guo, "Compatibility conditions from multipartite entanglement measures," Physical Review A, vol. 75, no. 5, Article ID 052324, 2007.

[116] M. Li, S.-M. Fei, X. Li-Jost, and Z.-X. Wang, Reports on Mathematical Physics. In press.

[117] L. Aolita, A. Buchleitner, and F. Mintert, "Scalable method to estimate experimentally the entanglement of multipartite systems," Physical Review A, vol. 78, no. 2, Article ID 022308, 2008.

[118] M. Li, S.-M. Fei, X. Li-Jost, and Z.-X. Wang, Chinese Physics B. In press.

[119] L. Masanes, "All bipartite entangled states are useful for information processing," Physical Review Letters, vol. 96, no. 15, Article ID 150501, 2006.

[120] G. Bowen and S. Bose, "Teleportation as a depolarizing quantum channel, relative entropy, and classical capacity," Physical Review Letters, vol. 87, no. 26, Article ID 267901, 2001.

[121] S. Albeverio, S.-M. Fei, and W.-L. Yang, "Teleportation with an arbitrary mixed resource as a tracepreserving quantum channel," Communications in Theoretical Physics, vol. 38, no. 3, pp. 301-304, 2002.

[122] J. Grondalski, D. M. Etlinger, and D. F. V. James, “The fully entangled fraction as an inclusive measure of entanglement applications," Physics Letters A, vol. 300, no. 6, pp. 573-580, 2002.

[123] M. Li, S.-M. Fei, and Z.-X. Wang, "Upper bound of the fully entangled fraction," Physical Review A, vol. 78, no. 3, Article ID 032332, 2008.

[124] R. F. Werner, "All teleportation and dense coding schemes," Journal of Physics A, vol. 34, no. 35, pp. 7081-7094, 2001.

[125] R.-J. Gu, M. Li, S.-M. Fei, and X.-Q. Li-Jost, “On estimation of fully entangled fraction," Communications in Theoretical Physics, vol. 53, no. 2, pp. 265-268, 2010.

[126] C.-S. Yu, X. X. Yi, and H.-S. Song, "Evolution of entanglement for quantum mixed states," Physical Review A, vol. 78, no. 6, Article ID 062330, 2008.

[127] M. Horodecki, P. Horodecki, and R. Horodecki, "Mixed-state entanglement and distillation: is there a "bound" entanglement in nature?" Physical Review Letters, vol. 80, no. 24, pp. 5239-5242, 1998.

[128] W. Du, J. I. Cirac, M. Lewenstein, and D. Bruß, "Distillability and partial transposition in bipartite systems," Physical Review A, vol. 61, no. 6, Article ID 062313, 2000.

[129] D. P. DiVincenzo, P. W. Shor, J. A. Smolin, B. M. Terhal, and A. V. Thapliyal, "Evidence for bound entangled states with negative partial transpose," Physical Review A, vol. 61, no. 6, Article ID 062312, 2000 . 


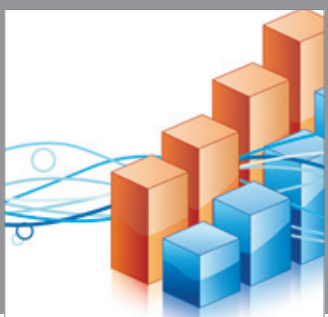

Advances in

Operations Research

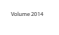

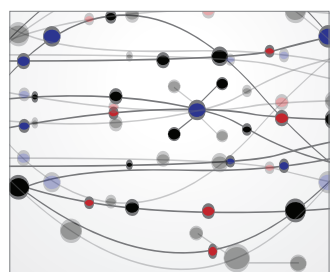

\section{The Scientific} World Journal
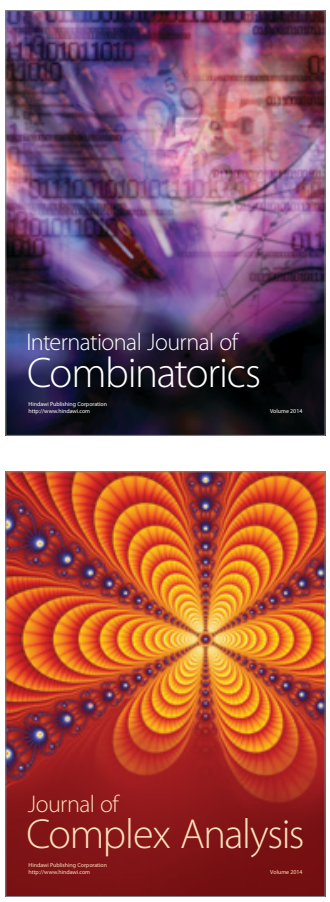

International Journal of

Mathematics and

Mathematical

Sciences
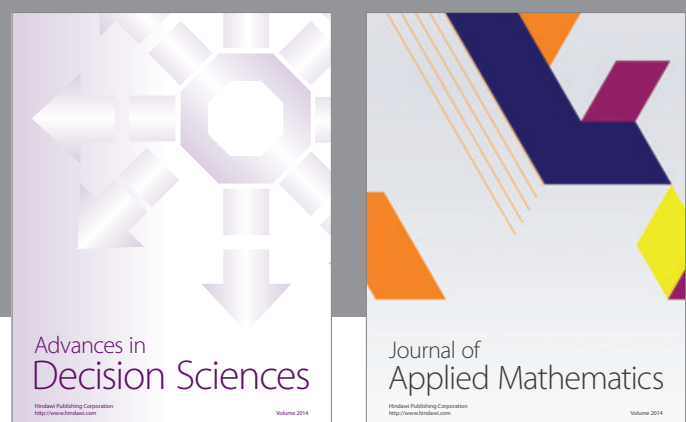

Journal of

Applied Mathematics
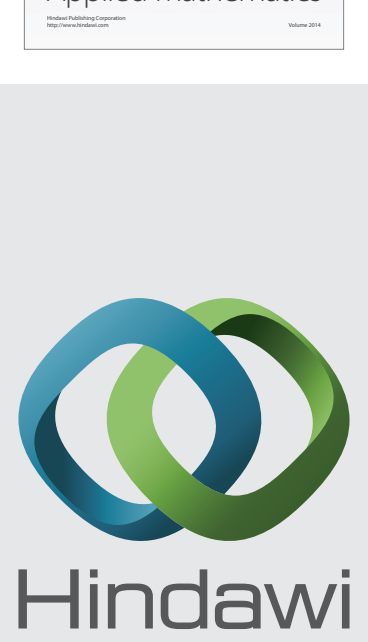

Submit your manuscripts at http://www.hindawi.com
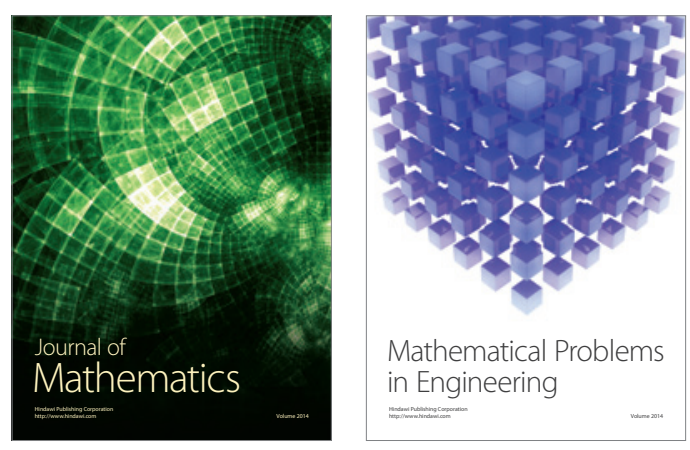

Mathematical Problems in Engineering
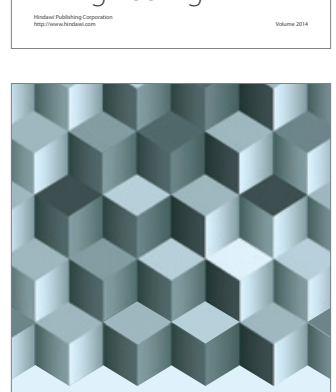

Journal of

Function Spaces
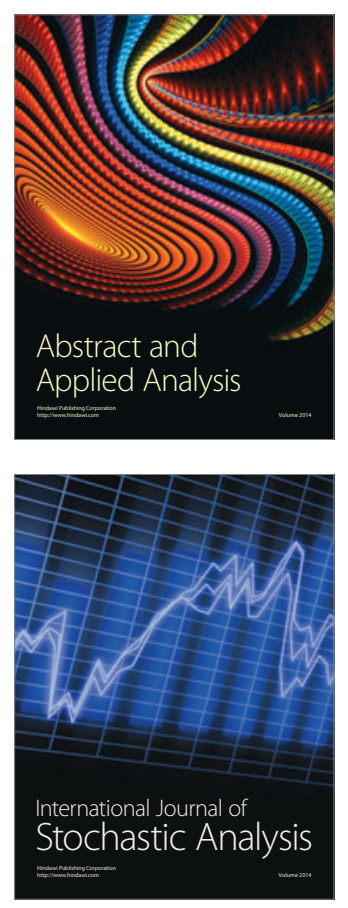

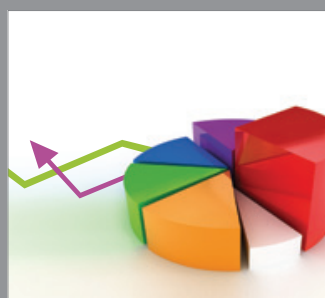

ournal of

Probability and Statistics

Promensencen
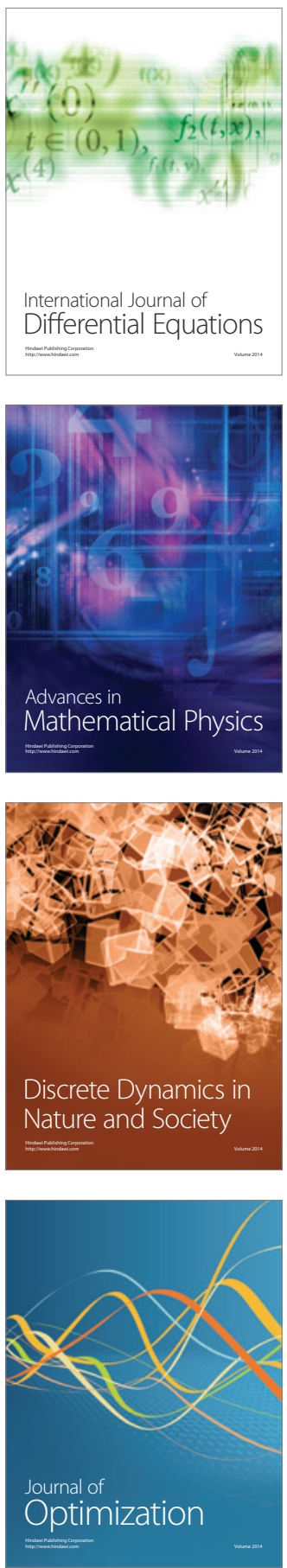